NBER WORKING PAPER SERIES

\title{
FINANCIAL MARKETS WHERE TRADERS NEGLECT THE INFORMATIONAL CONTENT OF PRICES
}

\author{
Erik Eyster \\ Matthew Rabin \\ Dimitri Vayanos \\ Working Paper 21224 \\ http://www.nber.org/papers/w21224
NATIONAL BUREAU OF ECONOMIC RESEARCH
1050 Massachusetts Avenue
Cambridge, MA 02138
May 2015

We thank Nick Barberis, John Campbell, Kent Daniel, Xavier Gabaix, Tristan Gagnon-Bartsch, Josh Schwartzstein, Andrei Shleifer, Jeremy Stein, seminar participants at Columbia, Humboldt, NYU Stern, UC Berkeley, UC Davis, and Wharton, and participants at the NBER Behavioral Finance Conference, the Paris School of Economics Workshop in Bounded Rationality, the Miami Behavioral Finance Conference, and the Stanford Institute for Theoretical Economics for helpful comments. We also thank Tristan Gagnon-Bartsch and Tarso Mori Madeira for research assistance. The views expressed herein are those of the authors and do not necessarily reflect the views of the National Bureau of Economic Research.

NBER working papers are circulated for discussion and comment purposes. They have not been peerreviewed or been subject to the review by the NBER Board of Directors that accompanies official NBER publications.

(C) 2015 by Erik Eyster, Matthew Rabin, and Dimitri Vayanos. All rights reserved. Short sections of text, not to exceed two paragraphs, may be quoted without explicit permission provided that full credit, including (C) notice, is given to the source. 
Financial Markets where Traders Neglect the Informational Content of Prices

Erik Eyster, Matthew Rabin, and Dimitri Vayanos

NBER Working Paper No. 21224

May 2015

JEL No. D53,D84,G02,G11,G12,G14

\begin{abstract}
$\underline{\text { ABSTRACT }}$
We present a model of a financial market where some traders are "cursed" when choosing how much to invest in a risky asset, failing to fully take into account what prices convey about others' private information. Cursed traders put more weight on their private signals than rational traders. But because they neglect that the price encodes other traders' information, prices depend less on private signals and more on public signals than rational-expectation-equilibrium (REE) prices. Markets comprised entirely of cursed traders generate more trade than those comprised entirely of rationals; mixed markets can generate even more trade, as rationals employ momentum-trading strategies to exploit cursed traders. We contrast our results to other models of departures from REE and show that per-trader volume with cursed traders increases when the market becomes large, while natural forms of overconfidence predict that volume should converge to zero.
\end{abstract}

Erik Eyster

The London School of Economics

Houghton St.

London WC2A 2AE, UK

eeyster@gmail.com

Matthew Rabin

Department of Economics

Littauer Center M-8

Harvard University

Cambridge, MA 02138

MatthewRabin@fas.harvard.edu
Dimitri Vayanos

Department of Finance, OLD 3.41

London School of Economics

Houghton Street

London WC2A 2AE

UNITED KINGDOM

and CEPR

and also NBER

d.vayanos@1se.ac.uk 


\section{Introduction}

People might rationally trade financial assets for a variety of non-speculative motives, such as portfolio rebalancing and liquidity. But ever since Milgrom and Stokey (1982), researchers have understood that common knowledge of rationality combined with a common prior precludes purely speculative trade. Even in settings where non-speculative motives enable speculation, a rational understanding of the adverse-selection problem causes the overall volume of trade to be constrained by non-speculative motives. In many people's estimation, trading volume in financial markets greatly exceeds what can be plausibly explained by models applying rational-expectations equilibrium $(\mathrm{REE}){ }^{1}$

Researchers have sought to explain excessive trading volume by relaxing the common-prior assumption. Harrison and Kreps (1978) show how non-common priors about an asset's payoff generate volume in a dynamic model where risk-neutral traders cannot sell the asset short. Scheinkman and Xiong (2003) use Harrison and Kreps' framework to explore traders who are "overconfident": all signals about the payoff are observed by all traders, yet certain traders overestimate the information content of certain signals. ${ }^{2}$ In these complete-information models, trade derives from traders agreeing to disagree about the relationship between future return and publicly available information. A second approach incorporates non-common priors into incomplete-information models by assuming that privately informed traders agree to disagree about the precision of traders' private information. Daniel, Hirshleifer and Subrahmanyam (1998, 2001) and Odean (1998), for example, show how traders' overconfidence about the precision of their private information can increase trad-

\footnotetext{
${ }^{1}$ For example, in his presidential address to the American Finance Association, French (2008) notes that the capitalized cost of trading exceeds $10 \%$ of market capitalization, and turnover in 2007 was $215 \%$, creating a puzzle that "[f]rom the perspective of the negative-sum game, it is hard to understand why equity investors pay to turn their aggregate portfolio over more than two times in 2007"' (page 1552).

${ }^{2}$ Hong, Scheinkman and Xiong (2006) model overconfidence similarly, allowing also for heterogenous priors, in a model where the number of shares of a risky asset increases over time due to the expiration of lock-up clauses.
} 
ing volume. ${ }^{3}$ Similarly, Odean (1998), Banerjee, Kaniel and Kremer (2009), Banerjee and Kremer (2010), and Banerjee (2011) show that when traders downplay the precision of one another's private signals - which we call contemptuousness - volume also increases.

Models of agreeing to disagree depict traders who recognize their disagreements in beliefs and trade based on them. This paper takes a different tack: people trade because they neglect disagreements in beliefs. This approach may seem retrograde, moving the theory of asset markets back to before the rational-expectations revolution. But it builds on evidence and modeling that people do not sufficiently attend to the information content of others' behavior - even in the absence of intrinsic disagreements. Laboratory evidence from settings with asymmetric information shows that people trade in ways that are consistent with disagreement neglect but that cannot be explained by non-common priors, overconfidence or contemptuousness. We present a simple and tractable model of markets where some or all traders, when choosing their demands, do not fully attend to the informational content of prices or others' trades. We draw out several implications that follow from this simple assumption, and contrast the model both to fully rational models and to existing alternatives to REE.

The most important implication is also the most basic: trading volume exceeds that of REE. Our model predicts that per-trader volume grows with the number of traders in the market. We contrast this result to markets where traders are overconfident and/or contemptuous but otherwise infer correctly from prices, in which case per-trader volume vanishes as the market becomes large. This suggests that disagreement neglect is a necessary condition for large volume in large markets: overconfidence and contemptuousness do not by themselves create large volume. ${ }^{4}$ We show additionally that disagreement neglect not only generates large volume, but also enables other biases

\footnotetext{
${ }^{3}$ Other models of trade deriving from differences in beliefs include Varian (1985), where traders have different subjective priors, DeLong, Shleifer, Summers, Waldmann (1990), where symmetrically informed traders disagree because some of them ("noise traders") misperceive next-period prices for exogenous reasons, as well as Harris and Raviv (1993) and Kandel and Pearson (1995), where traders disagree about the informativeness of public signals. Hong and Stein (2007) provide an overview of this literature.

${ }^{4}$ As argued earlier, large volume is hard to explain within REE settings as well.
} 
such as overconfidence and contemptuousness to matter in large markets.

Our model differs from REE not only in its predicted extent of trade, but also in its predictions about the qualitative properties of trading volume and prices. The price responds more to public information and less to private information than in REE. This implies that, unlike in REE, changes in asset prices are predictable from public information even in settings where traders lack non-speculative motives for trade such as random endowments. Returns are positively serially correlated when endowments are small — returns exhibit momentum-yet negatively correlated when endowments are large. Trading volume is hump-shaped in the precision of traders' private information, while (the smaller) volume in REE is inverse hump-shaped. Moreover, volume can be higher when some traders are rational and others neglect disagreement than when they all neglect disagreement. Also in contrast to REE, traders with more precise private information can be worse off than traders with less precise information.

Ours is not the first paper to use the assumption that some market participants fail to invert prices. Hong and Stein (1999) assume that some or all traders are "newswatchers" who trade based on news or signals they observe without inverting price to infer news that they haven't yet heard. They show how prices move predictably when information diffuses gradually through the market, similar to our result on momentum. Our work differs from theirs both in its formalization of the error traders make and in its applications. Conceptually, we relax Hong and Stein's assumption that traders infer nothing from market price to allow traders to partially infer from price, which we formalize in a general market-equilibrium concept. Content-wise, although Hong and Stein demonstrate the existence of volume, they do not explore the properties of volume nor compare its level to that predicted by alternative models such as overconfidence and contemptuousness.

Section 2 outlines the basic model upon which our approach builds. Based on evidence from strategic situations, Eyster and Rabin (2005) define cursed equilibrium in Bayesian games by the requirement that every player correctly predicts the behavior of others, but fails to fully attend to its informational content. Cursed equilibrium is meant to capture the intuitive psychology behind the "winner's curse" in common-values auctions, as well as related phenomena in other strategic 
settings. ${ }^{5}$ Section 2 then illustrates the workings of cursed equilibrium in a simple zero-sum game of speculative trade. We describe how the cursed-equilibrium prediction of trade in that setting is supported by direct experimental evidence. Moreover, that evidence cannot be accounted for by alternative approaches such as non-common priors, overconfidence or contemptuousness.

Section 3 introduces our formal set-up, based on Grossman (1976), Hellwig (1980) and Diamond and Verrechia (1981). We consider a market in which traders can exchange a risky for a riskless asset over one period. Traders observe public and private signals about the risky asset's payoff. We introduce a non-speculative motive to trade by assuming that traders receive random endowments correlated with the asset payoff. We define cursed-expectations equilibrium (CEE) by the requirement that some traders do not fully extract information from asset prices. CEE transplants the concept of cursed equilibrium from strategic games to price-taking competitive settings. As an application of a more general equilibrium concept, it has the methodological benefit of not having been designed specifically to explain the particular financial-market puzzles that we explore. For tractability, we assume that traders have constant-absolute-risk-aversion (CARA) preferences and that probability distributions are normal.

Section 4 analyzes the model without random endowments. As in Grossman (1976), there is a unique linear REE with the classical prediction of no trade, and a price that aggregates efficiently all public and private signals. We next solve for CEE when all traders are fully cursed, inferring nothing from price. Although each cursed trader relies too much upon his own private signal when estimating the asset payoff, because other traders fail to appreciate how that signal influences the price, private signals enter the price with a smaller weight than in REE. Conversely, the public signal enters the price with a larger weight because each trader relies on it more than in REE. These two effects make returns a predictable function of observables: future return depends positively on

\footnotetext{
${ }^{5}$ Cursedness explains the voluminous evidence on bidders succumbing to the "winner's curse" in commonvalues auctions - where the average price paid by the winner exceeds the average value of the object being auctioned - reviewed in Kagel and Levin (2002). Additional evidence consistent with cursedness comes from settings ranging from social learning (Weizsäcker (2010)), voting (Esponda and Vespa (2013)), and trade in positive-sum (Samuelson and Bazerman (1985), Holt and Sherman (1994)) and zero-sum (Carrillo and Palfrey (2011)) environments.
} 
past return and negatively on the public signal in a bivariate regression, and it depends positively on past return in a univariate regression. The positive coefficient on past return accords with the empirical evidence on momentum. ${ }^{6}$

We show additionally that traders with more precise private signals trade more than those with less precise ones, but can be worse off because cursedness causes them to trade too aggressively. The total volume of trade approaches infinity as the number of agents becomes large, and is humpshaped in the average precision of private signals. Intuitively, there is little private information when either signals are very imprecise - everybody knows very little - or when signals are very precise - everybody is fully informed.

We complete Section 4 by solving for CEE in two intermediate cases. We first find CEE when all traders are partially cursed to the same degree. The greater the extent of traders' cursedness, the more weight the public signal has and the less weight private signals have on price. The per-trader volume of trade increases in the number of market participants, whatever the level of traders' cursedness. Next, we consider markets where some traders are rational and others are fully cursed. Rational traders exploit the under-reaction of price to cursed traders' private signals by engaging in momentum trading, buying when the price goes up and selling when it goes down. Cursed traders take the other side of those trades. Because rational traders weight the private signals of cursed traders by inverting the price, whereas fully cursed traders do not weight the private signals of rational traders whatsoever, the precision-adjusted impact of the private signals of the cursed traders on prices exceeds that of the rational traders. Moreover, volume can be larger when some traders are rational and others fully cursed than when all are rational or all fully cursed. Intuitively, a cursed trader trades more against someone who does the opposite - a rational momentum trader - than against another cursed trader.

Section 5 analyzes the model with random endowments. As in Hellwig (1980) and Diamond and Verrechia (1981), trade happens even in REE. We show that in REE, the future return is a predictable, decreasing function of past return. When all traders are fully cursed, returns exhibit these same reversals if and only if endowment shocks are large enough. We show that REE trading

\footnotetext{
${ }^{6}$ See Jegadeesh and Titman (1993), and Jegadeesh and Titman (2011) for a recent survey.
} 
volume is inverse hump-shaped in the precision of private signals - the exact opposite result than when traders are cursed. ${ }^{7}$ Intuitively, adverse selection reduces volume when there are private signals, but disappears when everybody knows little due to imprecise signals or is fully informed because of very precise signals (Akerlof (1970), Hirshleifer (1971)).

Section 6 contrasts our approach and results to those of overconfidence-where traders exaggerate the precision of their own private signals - and contemptuousness - where traders downplay the precision of other traders' private signals. ${ }^{8}$ As in the literature, in the model of overconfidence and contemptuousness that we construct for comparison traders are otherwise rational: they understand the mapping between the price and other traders' private information-but they agree to disagree about the meaning of that information. We show that overconfidence leads to prices that depend too much on private information and too little on public information relative to REE - the exact opposite of our findings with cursed traders. Contemptuousness is a cousin of sorts to cursedness, and traders who downplay the informativeness of others' signals behave similarly to cursed ones. Traders who are overconfident or contemptuous trade too much.

More interesting is the contrast in the extent of over-trading as the market becomes large. Whereas the per-trader volume under cursedness increases with the number of traders, per-trader volume under overconfidence or contemptuousness converges to zero. Intuitively, while each trader thinks he knows more than he does, he understands that the total amount of "valid" information reflected in the price in a large market swamps his own information. Hence, the same no-trade logic that prevails in REE also prevails in large markets of overconfident or contemptuous traders. ${ }^{9}$

Disagreement neglect enables overconfidence to matter in large markets. We show that in

\footnotetext{
${ }^{7}$ Banerjee (2011) finds that contemptuousness predicts this same pattern relative to REE.

${ }^{8}$ This form of overconfidence is not exactly equivalent to people's sensation that they are more skilled than they are, as in the work of Malmendier and Tate (2005) on CEOs. Instead, it corresponds to people thinking "my private signals are better than they are," which might result from people thinking they are better than others at reading information, making it akin to ego-related overconfidence.

${ }^{9}$ Our finding that the per-trader volume of overconfident trade vanishes in large markets would also hold if all signals in the model were made public, which resembles the form of overconfidence assumed in Scheinkman and Xiong (2003).
} 
a large market of cursed overconfident traders, per-trader volume remains non-negligible. In this sense, cursedness and overconfidence work as complements, and cursedness helps vindicate the basic intuition from the literature that overconfidence can be a significant source of trading volume.

We conclude in Section 7. We discuss some of the limits of our model, especially the challenges in extending cursedness to multi-period settings. We also describe how cursedness may interact with other biases. In particular - as with overconfidence discussed in Section 6 - cursedness may serve as an "enabler" of various cognitive errors to which traders seem to fall prey: many errors matter significantly for prices and volume if and only if traders are cursed.

\section{Cursed Equilibrium}

In this section, we use a simple trading game to illustrate Eyster and Rabin's (2005) concept of cursed equilibrium, which underlies our concept of cursed-expectations equilibrium introduced in Section 3. In the context of this game, we show how cursed equilibrium differs from other theories that have been explored in the finance literature such as non-common priors, overconfidence and contemptuousness. A general definition of cursed equilibrium is in Appendix A.

The trading game works as follows. A seller owns an asset that he knows to be worth $s$ both to himself and to a potential buyer. The buyer does not know $s$, but believes that it is randomly drawn from $[0,1]$ with a cumulative distribution function $F$. The buyer makes the seller a take-itor-leave-it offer $p$ for the asset.

The seller's optimal strategy is to accept the buyer's offer $p$ if and only if $s \leq p$. In a Bayesian Nash equilibrium the buyer understands this, and so chooses $p$ to maximize $F(p) \times(E[s \mid s \leq p]-p)$. This objective is the probability $F(p)$ that the seller accepts the offer $p$, times the buyer's expected surplus from acquiring the asset conditional on seller acceptance. Because $E[s \mid s \leq p]<p$ for each $p>0$, the buyer's optimal offer is $p^{*}=0$. Thus, no trade occurs, consistent with Milgrom and Stokey's (1982) celebrated result on the absence of speculative trade between rational agents.

In a cursed equilibrium players fail to appreciate the informational content of others' behavior. This does not matter for the seller, who knows $s$ perfectly and hence has nothing to learn, but matters for the buyer. A buyer who is fully cursed completely neglects the relationship between the 
seller's willingness to sell at price $p$ and the seller's private information $s$, but correctly predicts the probability distribution over the seller's actions. As a consequence, a fully cursed buyer perceives the expected value of an asset traded at price $p$ to be its unconditional expectation, $E[s]$. A fully cursed buyer thus chooses $p$ to maximize $F(p) \times(\mathrm{E}[s]-p)$. A partially cursed buyer appreciates that the seller's willingness to sell correlates with his private information but underestimates that relationship. A buyer who is partially cursed with coefficient $\chi$ perceives the expected value of an asset traded at price $p$ to be $(1-\chi) \mathrm{E}[s \mid s \leq p]+\chi \mathrm{E}[s]$. This is the weighted average of the rational belief with weight $1-\chi$ and the fully cursed belief with weight $\chi$. In effect, the buyer believes that with probability $1-\chi$ the seller's decision to sell conveys information about the asset, and with probability $\chi$ it does not. The coefficient $\chi$ measures the buyer's naivety: $\chi=0$ corresponds to full rationality, while $\chi=1$ corresponds to full cursedness. A $\chi$-cursed buyer thus chooses $p$ to maximize $F(p) \times((1-\chi) \mathrm{E}[s \mid s \leq p]+\chi \mathrm{E}[s]-p)$. Since $\mathrm{E}[s]>0$, the buyer's optimal offer exceeds zero for any $\chi>0$. Moreover, since the buyer's objective function is supermodular in $(p, \chi)$ for $p \in[0, \mathrm{E}[s]]$, Topkis' Theorem implies that $p^{*}$ increases in $\chi$. In summary, cursedness produces trade in no-trade settings, and the more cursed the buyer, the higher the volume of trade.

Cursed trade derives from people's neglect of disagreement: the buyer neglects how the seller's willingness to trade at $p$ signals beliefs that the object is worth less than $p$. Researchers have proposed alternative models for speculative trade based on the idea that people are conscious of their disagreement and "agree to disagree." This is modelled as non-common priors about the distribution of asset payoffs, or as disagreement over the information that different signals convey about payoffs. Models of overconfidence assume a specific type of disagreement over signals: people believe that private signals they receive are more precise than private signals others receive. Such a belief may arise because people overestimate the precision of their signals while holding a correct assessment of the precision of others' signals. Alternatively, people may correctly assess the precision of their signals while underestimating the precision of others' signals. Researchers have considered both of these polar cases as well as their combination. We reserve the term overconfidence for the former case (people are overconfident about their ability to gather a precise signal), and use the term contemptuousness for the latter (people are contemptuous about others' ability). 
One way to identify cursedness in the data, and to distinguish from non-common priors, overconfidence and contemptuousness, is through laboratory experiments. Carrillo and Palfrey (2011) conduct experiments on a trading game that is a variant of the one considered in this section. The main difference is that in their game the buyer also has a private signal $b$, and the value of the object is some function $v(b, s)$. (The take-it-or-leave-it offer is also made by the seller rather than the buyer.) As in the game considered in this section, the Bayesian Nash equilibrium involves no trade but the cursed equilibrium does. Moreover, neither non-common priors, nor overconfidence, nor contemptuousness can generate trade. Carrillo and Palfrey argue indeed that behavior in their experiments is well explained by cursed equilibrium.

The failure of non-common priors, overconfidence and contemptuousness to generate trade can be seen in the game considered in this section (and the same logic transposes to Carrillo and Palfrey's game). Non-common priors about the distribution of asset payoffs would amount to the buyer and the seller employing different cumulative distribution functions $F$. This would not yield trade. Indeed, the seller has a dominant strategy that is independent of $F$, and the offer $p=0$ is optimal for the buyer regardless of his assessment of $F$. Overconfidence has no bite because the seller knows $s$ perfectly and so cannot exaggerate further the precision of his private information, while the buyer lacks private information whose precision to exaggerate. Contemptuousness would amount to the buyer believing that the seller has less information than he actually has, e.g., observes a noisy signal about $s$. Yet, the buyer would be unwilling to trade when the seller has private information no matter how imprecise that information is. Moreover, the information structure is announced clearly to all experiment participants.

In Section 6 we examine the relationship between cursedness, overconfidence and contemptuousness within our financial markets model. We show that cursedness yields distinct predictions concerning trading volume and its behavior in large markets.

\section{Model, Equilibrium Concept, and Equilibrium Conditions}

In this section, we begin by defining cursed-expectations equilibrium in a general version of our model, before making more specific assumptions on traders' cursedness coefficients, the distribution 
of their information, and their utility functions that allow us to derive analytically tractable, linear equilibria. There are two periods, 1 and 2, and two assets that pay off in terms of a consumption good in Period 2. One asset is riskless and pays off one unit of the consumption good with certainty. The other asset is risky and pays $d=\bar{d}+\epsilon+\zeta$ units, where $\bar{d}$ is a constant and $(\epsilon, \zeta)$ are random variables with mean zero. We use the riskless asset as the numeraire, and denote by $p$ the price of the risky asset in Period 1. Our choice of numeraire implies that the price of the risky asset in Period 2 is $d$ and the riskless rate is zero. We assume that the risky asset is in zero supply.

There are $N$ traders who can exchange the two assets in Period 1 . Trader $i=1, . ., N$ observes the private signal

$$
s_{i}=\epsilon+\eta_{i},
$$

as well as the public signal

$$
s=\epsilon+\theta,
$$

which is also observed by all other traders. The random variables $\left(\left\{\eta_{i}\right\}_{i=1, . ., N}, \theta\right)$ have mean zero. The signals are observed in Period 1. They provide information about the component $\epsilon$ of the risky asset's payoff but not about $\zeta$.

Trader $i$ starts with a zero endowment of the riskless and the risky assets, and receives an endowment $z_{i} d$ of the consumption good in Period 2. We refer to $z_{i}$ as the endowment shock, and assume that it is observed in Period 1 and has mean zero. Through its correlation with $d$, the endowment generates a hedging motive to trade. When, for example, $z_{i}>0$, trader $i$ is exposed to the risk that $d$ will be low and wishes to hedge by selling the risky asset. We assume that the variables $\left(\epsilon, \zeta,\left\{\eta_{i}\right\}_{i=1, . ., N}, \theta,\left\{z_{i}\right\}_{i=1, . ., N}\right)$ are mutually independent.

The budget constraint of trader $i$ is

$$
W_{i}=x_{i}(d-p)+z_{i} d
$$

where $x_{i}$ denotes the number of shares of the risky asset held by the trader in Period 1 . We impose no portfolio constraints, e.g., on short sales or leverage, and allow $x_{i}$ to take any value in $\mathbb{R}$. 
Traders maximize expected utility of consumption in Period 2. We denote by $u_{i}\left(W_{i}\right)$ the utility of trader $i$. If the trader is rational, he maximizes the expected utility

$$
\mathrm{E}\left[u_{i}\left(x_{i}(d-p)+z_{i} d\right) \mid\left\{s_{i}, s, z_{i}, p\right\}\right]
$$

where we use (3) to substitute for $W_{i}$. A rational trader conditions his estimate of the asset payoff $d$ on his private signal, the public signal, the endowment shock, and the price. A fully cursed trader completely neglects the relationship between the price and other traders' information, and maximizes the expected utility

$$
\mathrm{E}\left[u_{i}\left(x_{i}(d-p)+z_{i} d\right) \mid\left\{s_{i}, s, z_{i}\right\}\right]
$$

which differs from the rational expected utility because there is no conditioning on the price. To derive the objective of a partially cursed trader, we proceed as in Section 2 with a slight modification for tractability. Following the definition in Section 2, if trader $i$ is $\chi_{i}$-cursed, he maximizes the expected utility

$$
\left(1-\chi_{i}\right) \mathrm{E}\left[u_{i}\left(x_{i}(d-p)+z_{i} d\right) \mid\left\{s_{i}, s, z_{i}, p\right\}\right]+\chi_{i} \mathrm{E}\left[u_{i}\left(x_{i}(d-p)+z_{i} d\right) \mid\left\{s_{i}, s, z_{i}\right\}\right],
$$

which is the weighted average of the rational expected utility with weight $1-\chi_{i}$ and the fully cursed expected utility with weight $\chi_{i}$. For tractability, we employ the geometric average of utilities instead of the arithmetic average, hence modifying trader $i$ 's objective to

$$
\mathrm{E}\left[u_{i}\left(x_{i}(d-p)+z_{i} d\right) \mid\left\{s_{i}, s, z_{i}, p\right\}\right]^{1-\chi_{i}} \mathrm{E}\left[u_{i}\left(x_{i}(d-p)+z_{i} d\right) \mid\left\{s_{i}, s, z_{i}\right\}\right]^{\chi_{i}} .
$$

As in the case of the arithmetic average, $\chi_{i}=0$ corresponds to rationality, $\chi_{i}=1$ to full cursedness, and intermediate values of $\chi_{i}$ to traders who appreciate the information content of the price but only partially. The demand function of partially cursed traders always lies between the rational and the fully cursed demand functions.

Our definition of cursed-expectations equilibrium combines utility maximization under cursed expectations with market clearing. As in the case of REE, the equilibrium involves a price function $p$ that depends on all the random variables in the model. These are the private signals $\left\{s_{i}\right\}_{i=1, \ldots, N}$, the public signal $s$, and the endowment shocks $\left\{z_{i}\right\}_{i=1, \ldots, N}$. 
Definition 1 A price function $\left.p\left(\left\{s_{i}\right\}_{i=1, \ldots, N}, s,\left\{z_{i}\right\}_{i=1, \ldots, N}\right)\right)$ and demand functions $\left\{x_{i}\left(s_{i}, s, z_{i}, p\right)\right\}_{i=1, \ldots, N}$ are a cursed-expectations-equilibrium (CEE) if:

(i) (Optimization) For each trader $i=1, \ldots, N$, and each $\left(s_{i}, s, z_{i}, p\right)$,

$$
x_{i} \in \arg \max _{x}\left\{\mathrm{E}\left[u_{i}\left(x(d-p)+z_{i} d\right) \mid\left\{s_{i}, s, z_{i}, p\right\}\right]^{1-\chi_{i}} \mathrm{E}\left[u_{i}\left(x(d-p)+z_{i} d\right) \mid\left\{s_{i}, s, z_{i}\right\}\right]^{\chi_{i}}\right\},
$$

(ii) (Market Clearing) For each $\left(\left\{s_{i}\right\}_{i=1, \ldots, N}, s,\left\{z_{i}\right\}_{i=1, \ldots, N}\right)$,

$$
\sum_{i=1}^{N} x_{i}=0 .
$$

We next specialize our analysis by making two assumptions, which allow us to derive tractable linear equilibria. First, the variables $\left(\epsilon, \zeta,\left\{\eta_{i}\right\}_{i=1, . ., N}, \theta,\left\{z_{i}\right\}_{i=1, \ldots, N}\right)$ follow normal distributions, with variances denoted by $\left(\sigma_{\epsilon}^{2}, \sigma_{\zeta}^{2},\left\{\sigma_{\eta_{i}}^{2}\right\}_{i=1, . ., N}, \sigma_{\theta}^{2},\left\{\sigma_{z_{i}}^{2}\right\}_{i=1, . ., N}\right)$ and precisions, i.e., the inverses of the variances, denoted by $\left(\tau_{\epsilon}, \tau_{\zeta},\left\{\tau_{\eta_{i}}\right\}_{i=1, . ., N}, \tau_{\theta},\left\{\tau_{z_{i}}\right\}_{i=1, . ., N}\right)$. Second, traders have negative exponential, or constant absolute risk aversion (CARA), utility functions: $u_{i}\left(W_{i}\right)=-\exp \left(-\alpha_{i} W_{i}\right)$, where $\alpha_{i}$ is the coefficient of absolute risk aversion.

A linear CEE price function has the form

$$
p=\bar{d}+\sum_{i=1}^{N} A_{i} s_{i}+B s-\sum_{i=1}^{N} C_{i} z_{i},
$$

for coefficients $\left(\left\{A_{i}\right\}_{i=1, . . N}, B,\left\{C_{i}\right\}_{i=1, . . N}\right)$. For CARA utility, we can write the expectations in (4) as

$$
\begin{aligned}
& -\mathrm{E}\left[\exp \left[-\alpha_{i}\left(x_{i}(d-p)+z_{i} d\right)\right] \mid I_{i}\right] \\
& =-\exp \left[-\alpha_{i}\left(x_{i}\left(\mathrm{E}\left(d \mid I_{i}\right)-p\right)+z_{i} \mathrm{E}\left(d \mid I_{i}\right)-\frac{1}{2} \alpha_{i}\left(x_{i}+z_{i}\right)^{2} \operatorname{Var}\left(d \mid I_{i}\right)\right)\right],
\end{aligned}
$$

where the information set $I_{i}$ is equal to $I_{i r} \equiv\left\{s_{i}, s, z_{i}, p\right\}$ if $\chi_{i}=0$ (where $r$ stands for rational) and to $I_{i c} \equiv\left\{s_{i}, s, z_{i}\right\}$ if $\chi_{i}=1$ (where $c$ stands for fully cursed). The second step in (7) follows because all variables are normally distributed. Substituting (7) into (4) and maximizing, we find the demand function

$$
x_{i}=\frac{\left(1-\chi_{i}\right) \mathrm{E}\left(d \mid I_{i r}\right)+\chi_{i} \mathrm{E}\left(d \mid I_{i c}\right)-p}{\alpha_{i}\left[\left(1-\chi_{i}\right) \operatorname{Var}\left(d \mid I_{i r}\right)+\chi_{i} \operatorname{Var}\left(d \mid I_{i c}\right)\right]}-z_{i} .
$$


The demand function is the solution to a mean-variance problem. The trader's conditional expectation is the weighted average of the rational expectation with weight $1-\chi_{i}$ and the fully cursed expectation with weight $\chi_{i}$. The trader's conditional variance is the same weighted average of the rational and fully cursed variances. The geometric average formulation of utilities ensures that traders' optimization problems retain a tractable mean-variance structure even under partial cursedness. Combining (8) with the market-clearing condition (5), we derive conditions in Proposition 1 so that (6) is an equilibrium price. Proposition 1 does not show existence or uniqueness of $\left(\left\{A_{i}\right\}_{i=1, . . N}, B,\left\{C_{i}\right\}_{i=1, . . N}\right)$ satisfying these conditions, both of which are instead demonstrated in the special cases studied in subsequent sections.

To state Proposition 1, we introduce some notation. From the perspective of a rational trader $i$, the price (6) includes information on $\left(s_{i}, s, z_{i}\right)$, which the trader knows, and on $\left(\left\{s_{i}\right\}_{j \neq i},\left\{z_{i}\right\}_{j \neq i}\right)$, which he does not. The latter information is summarized in the signal

$$
\frac{\sum_{j \neq i} A_{j} s_{j}-\sum_{j \neq i} C_{j} z_{j}}{\sum_{j \neq i} A_{j}},
$$

which the trader can extract from the price. Using (1) and (2), we can write this signal as $\epsilon+\xi_{i}$, where

$$
\xi_{i} \equiv \frac{\sum_{j \neq i} A_{j} \eta_{j}-\sum_{j \neq i} C_{j} z_{j}}{\sum_{j \neq i} A_{j}} .
$$

We denote the variance of $\xi_{i}$ by $\sigma_{\xi_{i}}^{2}$ and its precision by $\tau_{\xi_{i}}$.

Proposition 1 The price (6) is an equilibrium price if and only if $\left(\left\{A_{i}\right\}_{i=1, . . N}, B,\left\{C_{i}\right\}_{i=1, . . N}\right)$ 
satisfy the conditions

$$
\begin{aligned}
& \quad \frac{\tau_{\eta_{i}}\left(\tau_{\epsilon}+\tau_{\eta_{i}}+\tau_{\theta}+\chi_{i} \tau_{\xi_{i}}\right)-\left(1-\chi_{i}\right) \tau_{\xi_{i}} \frac{A_{i}}{\sum_{k \neq i} A_{k}}\left(\tau_{\epsilon}+\tau_{\eta_{i}}+\tau_{\theta}\right)}{\alpha_{i}\left[\left(\tau_{\epsilon}+\tau_{\eta_{i}}+\tau_{\theta}\right)\left(\tau_{\epsilon}+\tau_{\eta_{i}}+\tau_{\theta}+\tau_{\xi_{i}}\right)+\tau_{\zeta}\left(\tau_{\epsilon}+\tau_{\eta_{i}}+\tau_{\theta}+\chi_{i} \tau_{\xi_{i}}\right)\right]} \\
& =A_{i} \sum_{j=1}^{N} \frac{\left(\tau_{\epsilon}+\tau_{\eta_{j}}+\tau_{\theta}\right)\left(\tau_{\epsilon}+\tau_{\eta_{j}}+\tau_{\theta}+\tau_{\xi_{j}}\right)-\left(1-\chi_{j}\right) \tau_{\xi_{j}} \frac{1}{\sum_{k \neq j} A_{k}}\left(\tau_{\epsilon}+\tau_{\eta_{j}}+\tau_{\theta}\right)}{\alpha_{j}\left[\left(\tau_{\epsilon}+\tau_{\eta_{j}}+\tau_{\theta}\right)\left(\tau_{\epsilon}+\tau_{\eta_{j}}+\tau_{\theta}+\tau_{\xi_{j}}\right)+\tau_{\zeta}\left(\tau_{\epsilon}+\tau_{\eta_{j}}+\tau_{\theta}+\chi_{j} \tau_{\xi_{j}}\right)\right]} \\
& \sum_{i=1}^{N} \frac{\tau_{\theta}\left(\tau_{\epsilon}+\tau_{\eta_{i}}+\tau_{\theta}+\chi_{i} \tau_{\xi_{i}}\right)}{\alpha_{i}\left[\left(\tau_{\epsilon}+\tau_{\eta_{i}}+\tau_{\theta}\right)\left(\tau_{\epsilon}+\tau_{\eta_{i}}+\tau_{\theta}+\tau_{\xi_{i}}\right)+\tau_{\zeta}\left(\tau_{\epsilon}+\tau_{\eta_{i}}+\tau_{\theta}+\chi_{i} \tau_{\xi_{i}}\right)\right]} \\
& =B \sum_{j=1}^{N} \frac{\left(\tau_{\epsilon}+\tau_{\eta_{j}}+\tau_{\theta}\right)\left(\tau_{\epsilon}+\tau_{\eta_{j}}+\tau_{\theta}+\tau_{\xi_{j}}\right)}{\alpha_{j}\left[\left(\tau_{\epsilon}+\tau_{\eta_{j}}+\tau_{\theta}\right)\left(\tau_{\epsilon}+\tau_{\eta_{j}}+\tau_{\theta}+\tau_{\xi_{j}}\right)+\tau_{\zeta}\left(\tau_{\epsilon}+\tau_{\eta_{j}}+\tau_{\theta}+\chi_{j} \tau_{\xi_{j}}\right)\right]} \\
& C_{i}=A_{i} \alpha_{i} \frac{\left(\tau_{\epsilon}+\tau_{\eta_{i}}+\tau_{\theta}\right)\left(\tau_{\epsilon}+\tau_{\eta_{i}}+\tau_{\theta}+\tau_{\xi_{i}}\right)+\tau_{\zeta}\left(\tau_{\epsilon}+\tau_{\eta_{i}}+\tau_{\theta}+\chi_{i} \tau_{\xi_{i}}\right)}{\tau_{\zeta} \tau_{\eta_{i}}\left(\tau_{\epsilon}+\tau_{\eta_{i}}+\tau_{\theta}+\chi_{i} \tau_{\xi_{i}}\right)}
\end{aligned}
$$

\section{No Endowment Shocks}

This section analyzes the model in the case where there are no endowment shocks. This case is derived by setting the variances $\left\{\sigma_{z_{i}}^{2}\right\}_{i=1, . ., N}$ of endowment shocks equal to zero. The shocks are then equal to their mean, which is zero. Without endowment shocks, traders lack a hedging motive to trade, and trade can occur only because of the private signals. The price (6) takes the form

$$
p=\bar{d}+\sum_{i=1}^{N} A_{i} s_{i}+B s .
$$

\subsection{All Traders Rational}

We begin with the benchmark case where all traders are rational $\left(\chi_{i}=0\right.$ for all $\left.i\right)$. Proposition 2 shows that the coefficients $\left(\left\{A_{i}\right\}_{i=1, . . N}, B\right)$ in the price (14), which are characterized by the conditions in Proposition 1, are uniquely determined.

Proposition 2 Suppose that there are no endowment shocks and all traders are rational. The price 
(14) is an equilibrium price if and only if

$$
\begin{gathered}
A_{i}=\frac{\tau_{\eta_{i}}}{\tau_{\epsilon}+\sum_{j=1}^{N} \tau_{\eta_{j}}+\tau_{\theta}}, \\
B=\frac{\tau_{\theta}}{\tau_{\epsilon}+\sum_{j=1}^{N} \tau_{\eta_{j}}+\tau_{\theta}} .
\end{gathered}
$$

There is no trade in equilibrium.

That there is no trade in equilibrium is a manifestation in the context of our model of the no-trade theorem of Milgrom and Stokey (1982). Since traders start with zero endowments in the risky asset and receive no endowment shocks, no-trade is a Pareto-efficient allocation and hence the unique equilibrium outcome.

The coefficients $\left(\left\{A_{i}\right\}_{i=1, . . N}, B\right)$ with which the private and the public signals enter into the price are proportional to these signals' precisions. Therefore, the price aggregates all the signals efficiently, as in Grossman (1976). The price equals the expected value of the asset payoff $d$ conditional upon all of the signals in the market.

\subsection{All Traders Fully Cursed}

We next turn to the case where all traders are fully cursed $\left(\chi_{i}=1\right.$ for all $\left.i\right)$. Proposition 3 shows that the coefficients $\left(\left\{A_{i}\right\}_{i=1, . . N}, B\right)$ in the price (14) are uniquely determined.

Proposition 3 Suppose that there are no endowment shocks and all traders are fully cursed. The price (14) is an equilibrium price if and only if

$$
\begin{aligned}
& A_{i}= \frac{\tau_{\eta_{i}}}{\alpha_{i}\left(\tau_{\epsilon}+\tau_{\zeta}+\tau_{\eta_{i}}+\tau_{\theta}\right)} \\
& \sum_{j=1}^{N} \frac{\tau_{\epsilon}+\tau_{\eta_{j}}+\tau_{\theta}}{\alpha_{j}\left(\tau_{\epsilon}+\tau_{\zeta}+\tau_{\eta_{j}}+\tau_{\theta}\right)} \\
& B=\frac{\sum_{j=1}^{N} \frac{\tau_{\theta}}{\alpha_{j}\left(\tau_{\epsilon}+\tau_{\zeta}+\tau_{\eta_{j}}+\tau_{\theta}\right)}}{\sum_{j=1}^{N} \frac{\tau_{\epsilon}+\tau_{\eta_{j}}+\tau_{\theta}}{\alpha_{j}\left(\tau_{\epsilon}+\tau_{\zeta}+\tau_{\eta_{j}}+\tau_{\theta}\right)}} .
\end{aligned}
$$

Unlike in the case where all traders are rational, the coefficients $\left(\left\{A_{i}\right\}_{i=1, . . N}, B\right)$ are not proportional to the precisions of the corresponding signals. Therefore, the price does not aggregate the signals efficiently. Proposition 4 compares the coefficients $\left(\left\{A_{i}\right\}_{i=1, . . N}, B\right)$ in the rational and fully cursed cases. 
Proposition 4 Suppose that there are no endowment shocks and all traders are fully cursed. Compared to the case where all traders are rational:

(i) The coefficient B with which the public signal enters into the price is larger.

(ii) The sum across traders of the coefficients $\left\{A_{i}\right\}_{i=1, . ., N}$ with which private signals enter into the price is smaller. The coefficient $A_{i}$ for any given trader $i$ can be larger or smaller.

(iii) The ratio $A_{i} / B$ is smaller.

Intuition for Proposition 4 and other results in this section can be derived from a general property of the price in the absence of endowment shocks. Substituting the demand functions $\left\{x_{i}\right\}_{i=1, . ., N}$ given by (8) into the market-clearing condition (5), setting $\left\{z_{i}\right\}_{i=1, . ., N}=0$, and solving for $p$, we find:

$$
p=\frac{\sum_{i=1}^{N} \frac{\left(1-\chi_{i}\right) \mathrm{E}\left(d \mid I_{i r}\right)+\chi_{i} \mathrm{E}\left(d \mid I_{i c}\right)}{\alpha_{i}\left[\left(1-\chi_{i}\right) \operatorname{Var}\left(d \mid I_{i r}\right)+\chi_{i} \operatorname{Var}\left(d \mid I_{i c}\right)\right]}}{\sum_{i=1}^{N} \frac{1}{\alpha_{i}\left[\left(1-\chi_{i}\right) \operatorname{Var}\left(d \mid I_{i r}\right)+\chi_{i} \operatorname{Var}\left(d \mid I_{i c}\right)\right]}} .
$$

The price is thus a weighted average of traders' conditional expectations of the asset payoff $d$, with trader $i$ 's conditional expectation receiving weight

$$
\frac{\frac{1}{\alpha_{i}\left[\left(1-\chi_{i}\right) \operatorname{Var}\left(d \mid I_{i r}\right)+\chi_{i} \operatorname{Var}\left(d \mid I_{i c}\right)\right]}}{\sum_{j=1}^{N} \frac{1}{\alpha_{j}\left[\left(1-\chi_{j}\right) \operatorname{Var}\left(d \mid I_{j r}\right)+\chi_{j} \operatorname{Var}\left(d \mid I_{j c}\right)\right]}} .
$$

The weight is larger for traders who are less risk averse or observe more precise signals. This is because they trade more aggressively on any given discrepancy between the price and their conditional expectation. In the rational case, all traders have the same conditional expectation because they learn from the price, which aggregates all the signals efficiently. Therefore, the price is also equal to that conditional expectation. In the fully cursed case, conditional expectations differ because traders do not learn other traders' signals from the price.

Since fully cursed traders form conditional expectations using fewer signals than rational traders, they attach larger weight to each signal they use. The public signal thus receives larger weight in each trader's conditional expectation than in the rational case, and hence enters the price with a larger coefficient (Result (i) of Proposition 4). The private signal of any given trader $i$ receives larger 
weight in that trader's conditional expectation but zero weight in all other traders' expectation. When trader $i$ 's risk aversion is low, the first effect dominates and that signal enters the price with a larger coefficient than in the rational case. The second effect dominates, however, when the coefficients are averaged across traders (Result (ii)). Note that when traders are symmetric, the coefficient corresponding to each trader equals the average coefficient, and hence is smaller than in the rational case. Finally, trader $i$ gives the correct weight to his private signal relative to the public signal. Because, however, other traders give zero weight to trader $i$ 's private signal but positive weight to the public signal, the price in the fully cursed case underweights the former signal relative to the latter (Result (iii)).

The results of Proposition 4 have implications for the predictability of asset returns. We define the return of the risky asset between Periods 1 and 2 as the difference between the asset payoff $d$ and the price $p$. We examine whether this return can be predicted using information available in Period 1, i.e., whether including such information improves the accuracy of a return forecast. When all traders are rational, the return is not predictable based on past information because $p$ is the expectation of $d$ conditional on all the signals available in Period 1.

Proposition 5 Suppose that there are no endowment shocks and all traders are fully cursed. The bivariate regression

$$
d-p=\gamma_{1}(p-\bar{d})+\gamma_{2} s+\nu
$$

yields coefficients $\gamma_{1}>0$ and $\gamma_{2}<0$. The univariate regressions

$$
\begin{aligned}
& d-p=\gamma s+\nu \\
& d-p=\gamma(p-\bar{d})+\nu
\end{aligned}
$$

yield coefficients $\gamma=0$ and $\gamma>0$, respectively. All regression coefficients are zero when all traders are rational.

The bivariate regression (20) predicts the asset return between Periods 1 and 2 using the public signal $s$ and the difference between the price $p$ and the unconditional expectation $\bar{d}$ of the asset 
payoff. The difference $p-\bar{d}$ can be interpreted as the return between a Period 0 , in which no signals are observed and the asset trades at $\bar{d}$, and Period 1. Hence, (20) measures the extent to which past return and the public signal predict future return. Since the price $p$ is influenced by the private and the public signals, and (20) controls for the latter, the coefficient $\gamma_{1}$ measures the effect of the private signals.

Because $\left(\gamma_{1}, \gamma_{2}\right)$ are non-zero, the return between Periods 1 and 2 is predictable. Holding the public signal constant, high private signals in Period 1 predict a price rise in Period $2\left(\gamma_{1}>0\right)$. Holding instead the private signals constant, a high public signal in Period 1 predicts a price drop $\left(\gamma_{2}<0\right)$. The price thus under-reacts to the private signals and over-reacts to the public signal. The under-reaction is consistent with Result (ii) of Proposition 4 that the average coefficient with which the private signals enter into the price is smaller in the fully cursed than in the rational case. The over-reaction is consistent with Result (i) that the coefficient with which the public signal enters into the price is larger in the fully cursed than in the rational case.

The univariate regressions (21) and (22) predict the asset return between Periods 1 and 2 using only the public signal $s$ or only the return between Periods 0 and 1 . The coefficient $\gamma$ in each of these regressions combines the effects of $\gamma_{1}$ and $\gamma_{2}$. Since $\gamma_{1}$ and $\gamma_{2}$ have opposite signs, the sign of $\gamma$ is a priori ambiguous.

Since $\gamma=0$ in the regression (21), the return between Periods 1 and 2 cannot be predicted using the public signal alone, even when traders are fully cursed. This stems from the fact that cursed traders process the limited set of signals that they observe correctly, and the public signal belongs to that set. The law of iterative expectations thus implies that when a trader's conditional expectation of the asset payoff $d$ is "conditioned down" on the public signal $s$, it must equal the expectation of $d$ conditional on $s$. Since the price $p$ equals a weighted average of traders' conditional expectations of $d$ (Eq. (19)), the expectation of $p$ conditional on $s$ equals that of $d$ conditional on $s$, and so $d-p$ cannot be predicted by $s$.

The return between Periods 1 and 2 can be predicted based on the return between Periods 0 and 1 only. Since $\gamma>0$, a high return between Periods 0 and 1 predicts a price rise in Period 2, which means that returns exhibit momentum. Since the price in Period 2 is equal to the asset's 
payoff, momentum in our model reflects price under-reaction to information. This under-reaction is driven by the price response to the private signals. Because the price under-reacts to information, the return between Periods 0 and 1 has lower variance than when traders are rational. Variance averaged across the return between Periods 0 and 1 and that between Periods 1 and 2 is also lower.

Corollary 1 Suppose that there are no endowment shocks and all traders are fully cursed. Compared to the case where all traders are rational, the variance of the return between Periods 0 and 1 is lower, and so is the variance averaged across the return between Periods 0 and 1 and that between Periods 1 and 2.

We next explore the implications of cursedness for trading volume. Since fully cursed traders do not learn others' signals from the price, they trade with each other even without endowment shocks. We define the trading volume generated by trader $i$ as the absolute value of the quantity $x_{i}$ that the trader trades in equilibrium. Proposition 6 computes expected trading volume in two special cases, and examines how it depends on the precision of private signals.

Proposition 6 Suppose that there are no endowment shocks and all traders are fully cursed.

(i) When all traders have the same risk-aversion coefficient $\alpha$ and observe private signals with the same precision $\tau_{\eta}$, the expected trading volume that each generates is

$$
\frac{\tau_{\zeta} \sqrt{2(N-1) \tau_{\eta}}}{\alpha\left(\tau_{\epsilon}+\tau_{\zeta}+\tau_{\eta}+\tau_{\theta}\right) \sqrt{\pi N}}
$$

Volume increases in the number of traders $N$. It is hump-shaped in the common precision $\tau_{\eta}$ of private signals, with the hump located at $\tau_{\eta}=\tau_{\epsilon}+\tau_{\zeta}+\tau_{\theta}$.

(ii) When all traders have the same risk-aversion coefficient $\alpha$ and the shock $\zeta$ has zero variance, the expected trading volume generated by trader $i$ is

$$
\frac{\sqrt{2\left[\left(\tau_{\epsilon}+\tau_{\theta}\right)^{2}\left[\tau_{\eta}+(N-2) \tau_{\eta_{i}}\right]+\left(\tau_{\epsilon}+\tau_{\theta}\right)\left[N \tau_{\eta}^{2}+(N-2) \tau_{\eta_{i}}^{2}\right]+\left(N \tau_{\eta}-\tau_{\eta_{i}}\right) \tau_{\eta} \tau_{\eta_{i}}\right]}}{\alpha\left(\tau_{\epsilon}+\tau_{\eta}+\tau_{\theta}\right) \sqrt{\pi N}},
$$

where $\tau_{\eta}$ denotes the average precision of private signals. Trader $i$ generates more volume than trader $j$ if and only if he observes a more precise private signal $\left(\tau_{\eta_{i}}>\tau_{\eta_{j}}\right)$. 
The intuition why trading volume is hump-shaped in the precision of private signals when traders are symmetric (Case (i) of Proposition 6) is as follows. Under symmetry, the quantity that a trader trades in equilibrium is equal to the difference between his conditional expectation of the asset payoff and the average conditional expectation of all traders, adjusted by his risk aversion and conditional variance. The difference in conditional expectations is zero when private signals have zero precision because traders then do not use them and share the same conditional expectation. It is also zero when private signals have infinite precision because they must then reveal the same information. For intermediate values of precision the dispersion in conditional expectations is positive, making volume hump-shaped in the common precision $\tau_{\eta}$ of private signals.

Adjusting by risk aversion and conditional variance preserves the hump-shaped pattern. The hump is located at a larger value of $\tau_{\eta}$, and so the adjustment causes volume to be increasing in $\tau_{\eta}$ over a larger interval. This is because an increase in signal precision reduces traders' conditional variances, hence making traders less uncertain and more eager to trade.

Case (ii) of Proposition 6 allows signal precision to differ across traders. Traders' conditional expectations can then differ both because of the private signals and because each trader weights the public signal differently when forming his expectation. For analytical simplicity we eliminate the shock $\zeta$ about which traders cannot learn by setting its variance to zero. For $\sigma_{\zeta}^{2}=0$, the hump in trading volume in Case (i) occurs for $\tau_{\eta}=\infty$, and so volume is always increasing in $\tau_{\eta}$. Proposition 6 shows that the same comparison applies in the cross section: traders who observe more precise signals generate more volume than those observing less precise signals.

When traders are symmetric, the per-trader volume increases in market size as measured by the number $N$ of traders. This is because the weight that each trader's private signal receives in the trader's conditional expectation is independent of $N$ while the weight that it receives in the price converges to zero when $N$ becomes large. Hence, each trader's beliefs become more discordant with price as $N$ increases. Since per-trader volume increases in $N$, aggregate volume converges to infinity when $N$ becomes large: cursedness produces large volume in large markets.

We finally compute traders' expected utilities and examine how they depend on the precision of private signals. We evaluate expected utility in the ex-ante sense, before signals appear (Period 
0 ). We also focus on the true expected utilities, i.e., compute the expectation under the true joint distribution of the signals and the price, rather than the distribution perceived by cursed traders.

Proposition 7 Suppose that there are no endowment shocks, all traders are fully cursed and have the same risk-aversion coefficient $\alpha$, and the shock $\zeta$ has zero variance. The expected utility of trader $i$ is

$$
-\frac{1}{\sqrt{1+\frac{\left(\tau_{\epsilon}+\tau_{\theta}\right)^{2}\left[N \tau_{\eta_{i}}-(2 N-1) \tau_{\eta}\right]-\left(\tau_{\epsilon}+\tau_{\theta}\right)\left[N(2 N-1) \tau_{\eta}^{2}-2 N(N+1) \tau_{\eta} \tau_{\eta_{i}}+(N-2) \tau_{\eta_{i}}^{2}\right]-\left(N \tau_{\eta}-\tau_{\eta_{i}}\right) \tau_{\eta} \tau_{\eta_{i}}}{N\left(\tau_{\epsilon}+\tau_{\eta}+\tau_{\theta}\right)^{2}\left(\tau_{\epsilon}+N \tau_{\eta}+\tau_{\theta}\right)}}},
$$

where $\tau_{\eta}$ denotes the average precision of private signals. A trader $i$ who observes a more precise signal than a trader $j\left(\tau_{\eta_{i}}>\tau_{\eta_{j}}\right)$ has higher expected utility if

$$
\left(\tau_{\epsilon}+\tau_{\theta}\right)^{2}+2(N+1)\left(\tau_{\epsilon}+\tau_{\theta}\right) \tau_{\eta}-\tau_{\eta}^{2}>0
$$

When (26) fails, there exist $\tau_{\eta_{i}}>\tau_{\eta_{j}}$ such that trader $i$ has lower expected utility than trader $j$.

Proposition 7 shows that fully cursed traders who observe more precise signals can be worse off relative to those observing less precise signals. This is not a manifestation of the standard result that asymmetric information makes traders worse off by destroying trading opportunities (Akerlof (1970), Hirshleifer (1971)). Indeed, the standard result compares expected utilities when traders are uninformed to those when some traders become informed and equilibrium prices change. Proposition 7 compares instead expected utilities of two traders who differ in the precision of their information, are present in the market at the same time, and face the same equilibrium price.

To explain how signal precision affects expected utility, we consider a trader who observes a completely uninformative private signal. Because the trader does not learn from the price, he falls victim to trading against others' private signals, e.g., buys when others observe negative signals. If the trader observes instead an informative signal, less of that trading occurs because his signal is better aligned with others' signals, e.g., is more likely to be negative when others' signals are negative. At the same time, a new effect appears: because the trader does not learn from the price, he trades overly aggressively on his signal and takes on excessive risk. When the second effect dominates, traders with more precise signals are worse off relative to those with less precise signals. 
According to (26), the second effect dominates when the average precision $\tau_{\eta}$ of private signals is high, but the first effect dominates when the number $N$ of traders is large. Intuitively, when precision is high, signals are essentially identical and information is symmetric. As a consequence, returns are not predictable (the regression coefficients in Proposition 5 are zero) and trading against others' signals does not generate a loss. Hence, the first effect disappears. When instead the market is large, returns remain predictable. Indeed, even though the price aggregates a large number of signals and provides highly precise information, cursed traders neglect that information and rely only on their own signals.

\subsection{Intermediate Cases}

We next analyze two intermediate cases between the polar extremes of rationality and full cursedness studied in the previous sections.

\subsubsection{Partially Cursed Traders}

The first intermediate case is when all traders are partially cursed with the same cursedness coefficient $\chi$. For analytical simplicity, we assume that traders also have the same risk-aversion coefficient $\alpha$ and observe private signals with the same precision $\tau_{\eta}$. Thus, the price (14) takes the form

$$
p=\bar{d}+A \sum_{i=1}^{N} s_{i}+B s .
$$

Proposition 9 shows that the coefficients $(A, B)$ are uniquely determined, and examines how these coefficients as well as trading volume depend on $\chi$.

Proposition 8 Suppose that there are no endowment shocks and all traders are partially cursed with the same cursedness coefficient $\chi$, risk aversion coefficient $\alpha$, and private signal precision $\tau_{\eta}$. The price (27) is an equilibrium price if and only if

$$
\begin{aligned}
& A=\frac{\tau_{\eta}\left\{[N-(N-1) \chi]\left(\tau_{\epsilon}+\tau_{\theta}\right)+N \tau_{\eta}\right\}}{N\left(\tau_{\epsilon}+\tau_{\eta}+\tau_{\theta}\right)\left(\tau_{\epsilon}+N \tau_{\eta}+\tau_{\theta}\right)}, \\
& B=\frac{\tau_{\theta}\left\{\tau_{\epsilon}+[1+\chi(N-1)] \tau_{\eta}+\tau_{\theta}\right\}}{\left(\tau_{\epsilon}+\tau_{\eta}+\tau_{\theta}\right)\left(\tau_{\epsilon}+N \tau_{\eta}+\tau_{\theta}\right)}
\end{aligned}
$$


The coefficient $A$ decreases in $\chi$ and the coefficient $B$ increases in $\chi$. The expected trading volume that each trader generates is

$$
\frac{\chi \tau_{\zeta}\left(\tau_{\epsilon}+N \tau_{\eta}+\tau_{\theta}\right) \sqrt{2(N-1) \tau_{\eta}}}{\alpha\left(\tau_{\zeta}\left\{\tau_{\epsilon}+[1+\chi(N-1)] \tau_{\eta}+\tau_{\theta}\right\}+\left(\tau_{\epsilon}+\tau_{\eta}+\tau_{\theta}\right)\left(\tau_{\epsilon}+N \tau_{\eta}+\tau_{\theta}\right)\right) \sqrt{\pi N}}
$$

Volume increases in $\chi$ and $N$.

As traders become more cursed ( $\chi$ increases), the private signals enter the price with a smaller coefficient $A$, the public signal enters with a larger coefficient $B$, and trading volume increases. The results on $(A, B)$ are consistent with the comparisons between rationality and full cursedness derived in Proposition 4. Note that per-trader volume under partial cursedness increases in the number $N$ of traders. This result, which also holds under full cursedness, implies that aggregate volume converges to infinity when $N$ becomes large.

\subsubsection{Rational and Fully Cursed Traders}

The second intermediate case is when some traders are rational and some are fully cursed. We denote by $N_{r}$ and $N_{c}$, respectively, the number of rational and fully cursed traders, and by $R$ and $C$ the sets of these traders. For analytical simplicity we assume that traders within each group have the same risk-aversion coefficient and private-signal precision. We denote by $\alpha_{r}$ and $\tau_{\eta r}$ the risk-aversion coefficient and private-signal precision for a rational trader, and by $\alpha_{c}$ and $\tau_{\eta c}$ the same quantities for a fully cursed trader. The price (14) takes the form

$$
p=\bar{d}+A_{r} \sum_{i \in R} s_{i}+A_{c} \sum_{i \in C} s_{i}+B s .
$$

Proposition 9 shows that the coefficients $\left(A_{r}, A_{c}, B\right)$ are uniquely determined.

Proposition 9 Suppose that there are no endowment shocks, and there are $N_{r}$ rational traders with risk-aversion coefficient $\alpha_{r}$ and private-signal precision $\tau_{\eta r}$, and $N_{c}$ fully cursed traders with risk-aversion coefficient $\alpha_{c}$ and private-signal precision $\tau_{\eta c}$. The price (31) is an equilibrium price 
if and only if

$$
\begin{aligned}
A_{r}= & x A_{c}, \\
A_{c}= & \frac{\frac{N_{r} \tau_{\xi}}{\alpha_{r}\left[\left(N_{r}-1\right) x+N_{c}\right]\left(\tau_{\epsilon}+\tau_{\zeta}+\tau_{\eta r}+\tau_{\theta}+\tau_{\xi}\right)}+\frac{\tau_{\eta c}}{\alpha_{c}\left(\tau_{\epsilon}+\tau_{\zeta}+\tau_{\eta c}+\tau_{\theta}\right)}}{\frac{N_{r}\left(\tau_{\epsilon}+\tau_{\eta r}+\tau_{\theta}+\tau_{\xi}\right)}{\alpha_{r}\left(\tau_{\epsilon}+\tau_{\zeta}+\tau_{\eta r}+\tau_{\theta}+\tau_{\xi}\right)}+\frac{N_{c}\left(\tau_{\epsilon}+\tau_{\eta c}+\tau_{\theta}\right)}{\alpha_{c}\left(\tau_{\epsilon}+\tau_{\zeta}+\tau_{\eta c}+\tau_{\theta}\right)}}, \\
B= & \frac{\frac{N_{r} \tau_{\theta}}{\alpha_{r}\left(\tau_{\epsilon}+\tau_{\zeta}+\tau_{\eta r}+\tau_{\theta}+\tau_{\xi}\right)}+\frac{N_{c} \tau_{\theta}}{N_{c}\left(\tau_{\epsilon}+\tau_{\zeta}+\tau_{\eta c}+\tau_{\theta}\right)}}{\frac{N_{r}\left(\tau_{\epsilon}+\tau_{\eta r}+\tau_{\theta}+\tau_{\xi}\right)}{\alpha_{r}\left(\tau_{\epsilon}+\tau_{\zeta}+\tau_{\eta r}+\tau_{\theta}+\tau_{\xi}\right)}+\frac{N_{c}\left(\tau_{\epsilon}+\tau_{\eta c}+\tau_{\theta}\right)}{\alpha_{c}\left(\tau_{\epsilon}+\tau_{\zeta}+\tau_{\eta c}+\tau_{\theta}\right)}}
\end{aligned}
$$

where

$$
\tau_{\xi} \equiv \frac{\left[\left(N_{r}-1\right) x+N_{c}\right]^{2} \tau_{\eta r} \tau_{\eta c}}{\left(N_{r}-1\right) x^{2} \tau_{\eta c}+N_{c} \tau_{\eta r}}
$$

and $x \in\left(0, \frac{\tau_{\eta r}}{\tau_{\eta c}}\right)$ is the unique solution of

$$
x=\frac{\alpha_{c} N_{c} \tau_{\eta r}\left(\tau_{\eta r}-x \tau_{\eta c}\right)\left(\tau_{\epsilon}+\tau_{\zeta}+\tau_{\eta c}+\tau_{\theta}\right)}{\alpha_{r} \tau_{\eta c}\left\{\left[\left(N_{r}-1\right) x^{2} \tau_{\eta c}+N_{c} \tau_{\eta r}\right]\left(\tau_{\epsilon}+\tau_{\zeta}+\tau_{\eta r}+\tau_{\theta}\right)+\left[\left(N_{r}-1\right) x+N_{c}\right]^{2} \tau_{\eta r} \tau_{\eta c}\right\}} .
$$

Since the fully cursed traders are symmetric, their private signals enter the price with the same weight, and equal weighting is efficient because the signals have the same precision. At the same time, the price gives too much weight to the signals of the fully cursed traders relative to those of the rational traders. Indeed, since $x=\frac{A_{r}}{A_{c}}<\frac{\tau_{\eta r}}{\tau_{\eta c}}$, the precision-adjusted coefficient $\frac{A_{r}}{\tau_{\eta r}}$ with which the rational traders' signals enter the price is smaller than its counterpart $\frac{A_{c}}{\tau_{\eta c}}$ for fully cursed traders. The intuition is that conditional expectations of rational traders give weight to both their own signals and those of fully cursed traders, while conditional expectations of fully cursed traders give weight to their own signals only.

We next characterize the trading activity of rational and fully cursed traders, and do so by regressing their signed volume on past return. A positive regression coefficient means that a trader buys following a price rise, and is hence a momentum trader. A negative coefficient means instead that a trader buys following a price decline. When traders are symmetric (all rational or all fully cursed), the regression coefficients are zero for all traders because of symmetry and because aggregate signed volume is zero. When instead some traders are rational and some are fully cursed, the regression coefficients are non-zero. Recall from Proposition 5 that returns in a market with fully 
cursed traders exhibit momentum because the price under-reacts to the private signals. Rational traders exploit this predictability, buying when the price goes up, hence acting as momentum traders. Fully cursed traders, by contrast, do the opposite because they neglect that high prices signal positive private information.

Proposition 10 Suppose that there are no endowment shocks, and there are $N_{r}$ rational traders with risk-aversion coefficient $\alpha_{r}$ and private-signal precision $\tau_{\eta r}$, and $N_{c}$ fully cursed traders with risk-aversion coefficient $\alpha_{c}$ and private-signal precision $\tau_{\eta c}$. The univariate regression

$$
x_{i}=\gamma_{i}(p-\bar{d})+\nu
$$

yields coefficient $\gamma_{i}>0$ for $i \in R$ and $\gamma_{i}<0$ for $i \in C$.

Because momentum trading adds to trading volume, volume can be higher in a market where some traders are rational and some are fully cursed than in an otherwise identical market where all traders are fully cursed. To show this result, we hold constant the total number $N$ of traders, assume that they all have the same risk-aversion coefficient $\left(\alpha_{r}=\alpha_{c}=\alpha\right)$ and private-signal precision $\left(\tau_{\eta r}=\tau_{\eta c}=\tau_{\eta}\right)$, and change the relative numbers of rational and fully cursed traders. We compute expected aggregate trading volume, defined as the sum over all traders of the expected trading volume that each generates.

Proposition 11 Suppose that there are no endowment shocks, and there are $N$ traders with riskaversion coefficient $\alpha$ and private-signal precision $\tau_{\eta c}$. Expected aggregate trading volume is larger when one trader is rational and $N-1$ are fully cursed than when all $N$ traders are fully cursed, under the sufficient condition

$$
\frac{1}{N-1}\left(\frac{\tau_{\epsilon}+N \tau_{\eta}+\tau_{\theta}}{\tau_{\epsilon}+\tau_{\eta}+\tau_{\theta}}\right)^{2}+\left(\frac{2\left(\tau_{\epsilon}+\tau_{\zeta}+N \tau_{\eta}+\tau_{\theta}\right)}{(N-1)\left(\tau_{\epsilon}+\tau_{\zeta}+\tau_{\eta}+\tau_{\theta}\right)}-1\right) \frac{\tau_{\epsilon}+N \tau_{\eta}+\tau_{\theta}}{\tau_{\epsilon}+\tau_{\eta}+\tau_{\theta}}+\left(\frac{\tau_{\epsilon}+\tau_{\zeta}+N \tau_{\eta}+\tau_{\theta}}{\tau_{\epsilon}+\tau_{\zeta}+\tau_{\eta}+\tau_{\theta}}\right)^{2}<0 .
$$

When the shock $\zeta$ has zero variance, (38) is satisfied if $N$ exceeds a threshold $\bar{N}$.

When (38) is satisfied, volume is hump-shaped in the number of rational traders: it increases when that number increases from zero to one, and it decreases to zero when rational traders make 
up all of the market. The increasing region can exist if the number $N$ of traders is large: enough cursed traders are needed so that return predictability is important and induces rational traders to engage in a sizeable amount of momentum trading. Moreover, volume can increase in the number of rational traders even when that number is well above one. Indeed, our numerical calculations indicate that the hump of trading volume can occur at a point where rational traders make up about half of the market.

Taken together, Propositions 8 and 11 imply that a market with some rational and some fully cursed traders is not equivalent in terms of volume to a market with partially cursed traders. Indeed, volume in the latter market is smaller than volume with all traders fully cursed (Proposition 8), which in turn can be smaller than volume in the former market (Proposition 11).

\section{$5 \quad$ Endowment Shocks}

In this section we introduce endowment shocks. With endowment shocks, the volume generated by rational traders becomes non-zero and returns become predictable. We derive these properties and compare with the case where traders are fully cursed. For analytical simplicity, we assume that traders have the same risk aversion coefficient $\alpha$, private-signal precision $\tau_{\eta}$, and endowment-shock precision $\tau_{z}$. Thus, the price (14) takes the form

$$
p=\bar{d}+A \sum_{i=1}^{N} s_{i}+B s-C \sum_{i=1}^{N} z_{i} .
$$

Proposition 12 determines the coefficients $(A, B, C)$ when all traders are rational and when they are all fully cursed.

Proposition 12 Suppose that there are endowment shocks and all traders have the same riskaversion coefficient $\alpha$, private-signal precision $\tau_{\eta}$, and endowment-shock precision $\tau_{z}$. When all 
traders are rational, the price (39) is an equilibrium price if and only if

$$
\begin{aligned}
& A=\frac{\tau_{\eta}+\frac{(N-1) \tau_{\eta} \tau_{z}}{\tau_{z}+\frac{C^{2}}{A^{2}} \tau_{\eta}}}{N\left(\tau_{\epsilon}+\tau_{\eta}+\tau_{\theta}+\frac{(N-1) \tau_{\eta} \tau_{z}}{\tau_{z}+\frac{C^{2}}{A^{2}} \tau_{\eta}}\right)}, \\
& B=\frac{\tau_{\theta}}{\tau_{\epsilon}+\tau_{\eta}+\tau_{\theta}+\frac{(N-1) \tau_{\eta} \tau_{z}}{\tau_{z}+\frac{C^{2}}{A^{2}} \tau_{\eta}}},
\end{aligned}
$$

and $\frac{C}{A}>0$ is the unique solution to the cubic equation

$$
\left(\tau_{z}+\frac{C^{2}}{A^{2}} \tau_{\eta}\right)\left(\frac{C}{A} \tau_{\zeta} \tau_{\eta}-\alpha\left(\tau_{\epsilon}+\tau_{\zeta}+\tau_{\eta}+\tau_{\theta}\right)\right)-\alpha(N-1) \tau_{\eta} \tau_{z}=0
$$

When all traders are fully cursed, $(A, B, C)$ are derived from (40)-(42) by setting $\tau_{z}=0$.

When all traders are fully cursed, the coefficients $A$ on the private signals and $B$ on the public signal do not depend on the precision $\tau_{z}$ of the endowment shocks. Indeed, because fully cursed traders do not learn from the price, $\tau_{z}$ does not affect the way that the signals and the price enter into their demand function. The coefficients $(A, B)$ for rational traders coincide with those for fully cursed traders when the endowment shocks have infinite variance $\left(\tau_{z}=0\right)$. This is because for $\tau_{z}=0$ the price becomes uninformative, and hence rational traders have the same demand function as fully cursed traders. When instead the endowment shocks have zero variance $\left(\tau_{z}=\infty\right)$, the coefficients $(A, B)$ for rational traders coincide with their counterparts in Proposition 2.

Endowment shocks do not change the result of Section 4 that the return between Periods 1 and 2 cannot be predicted using the public signal alone. That return, however, becomes predictable using the return between Periods 0 and 1 even when traders are rational. Returns with rational traders and endowment shocks exhibit reversals: a high return between Periods 0 and 1 predicts a price decline in Period 2. If the variance of endowment shocks is large enough, returns with fully cursed traders exhibit reversals as well, otherwise they exhibit momentum as in Section 4 . Endowment shocks tend to generate reversals because they cause prices to deviate temporarily from traders' expectations of the asset payoff.

Proposition 13 Suppose that there are endowment shocks and all traders have the same riskaversion coefficient $\alpha$, private-signal precision $\tau_{\eta}$, and endowment-shock precision $\tau_{z}$. When all 
traders are rational, the univariate regression (22) yields coefficient $\gamma<0$. When all traders are fully cursed, (22) yields coefficient $\gamma<0$ if and only if

$$
\frac{\alpha^{2}\left(\tau_{\epsilon}+\tau_{\zeta}+\tau_{\eta}+\tau_{\theta}\right)^{2}}{(N-1) \tau_{\zeta}^{2} \tau_{\eta} \tau_{z}}>1
$$

The univariate regression (21) yields coefficient $\gamma=0$ under both rationality and full cursedness.

Endowment shocks generate trading volume even when traders are rational. Rational volume, however, has different qualitative properties than cursed volume. Volume with fully cursed traders and no endowment shocks is hump-shaped in the precision of private signals, as shown in Section 4, and this property extends to endowment shocks. Volume with rational traders, however, is inverse hump-shaped in private-signal precision. The intuition in the rational case is as follows. Starting from the case where precision is zero, an increase in precision reduces volume because it introduces adverse selection (Akerlof (1970), Hirshleifer (1971)). Adverse selection disappears again when precision is infinite because traders' signals are identical.

Proposition 14 Suppose that there are endowment shocks and all traders have the same riskaversion coefficient $\alpha$, private-signal precision $\tau_{\eta}$, and endowment-shock precision $\tau_{z}$. The expected volume that each trader generates increases in the number of traders $N$ both when all traders are rational and when they are fully cursed. Volume is inverse hump-shaped in the common precision $\tau_{\eta}$ of private signals when all traders are rational, and is hump-shaped when they are fully cursed.

When the shock $\zeta$ about which traders cannot learn has zero variance, the hump in volume with rational traders occurs for $\tau_{\eta}=\infty$, and so volume is always decreasing in $\tau_{\eta}$. Intuitively, when precision is infinite, traders know the asset payoff perfectly: while there is no adverse selection, there are also no gains from risk-sharing. Notice that when $\zeta$ has zero variance, the corresponding hump with fully cursed traders also occurs for $\tau_{\eta}=\infty$, and so volume is always increasing in $\tau_{\eta}$.

\section{Overconfidence and Contemptuousness}

In this section we examine the relationship between cursedness, overconfidence and contemptuousness. We introduce overconfidence and contemptuousness into our model following Odean (1998), 
like him specializing our model to no endowment shocks and to traders who are symmetric in terms of their risk-aversion coefficient $\alpha$ and private signal precision $\tau_{\eta}$. Ruling out endowment shocks allows us to focus on trading volume that is purely driven by traders' cognitive errors.

Following Odean, we capture overconfidence by assuming that each trader incorrectly perceives the precision of his own signal to be $\kappa \times \tau_{\eta}$, for $\kappa \geq 1$ (and $\kappa=1$ embedding REE in our analysis below), but correctly perceives the precision of all other traders' signals to be $\tau_{\eta}$. Moreover, this belief system is common knowledge: it is common knowledge that each trader thinks he is better informed than all other traders think he is. We also follow Odean by allowing traders to err in a second, conceptually distinct way, namely by underestimating the information content in others' private signals, which we term contemptuousness. Each trader incorrectly perceives the precision of all other traders' signals to be $\gamma \times \tau_{\eta}$, for $\gamma \in[0,1]$ (and $\gamma=1$, together with $\kappa=1$, embedding REE in our analysis below). This too is common knowledge.

Under the parameter values $\kappa=1$ and $\gamma=0$, i.e., no overconfidence and extreme contemptuousness, behavior is the same as under full cursedness $(\chi=1)$. This is because a trader who fails to infer another's signal from the price behaves identically to one who fully infers that signal but erroneously assumes that it has zero precision. In the rest of this section we identify contemptuousness with $\gamma>0$, i.e., traders do not view others' signals as completely worthless. We contrast such non-extreme contemptuousness $(\gamma \in(0,1))$ with full or partial cursedness $(\chi \in(0,1])$.

Because of symmetry, the price takes the form

$$
p=\bar{d}+A \sum_{i=1}^{N} s_{i}+B s .
$$

Proposition 15 shows that the coefficients $(A, B)$ are uniquely determined, and examines how they depend on the overconfidence parameter $\kappa$ and the contemptuousness parameter $\gamma$.

Proposition 15 Suppose that there are no endowment shocks and all traders have the same riskaversion coefficient $\alpha$ and private-signal precision $\tau_{\eta}$. Suppose also that each trader misperceives the precision of his private signal to be $\kappa \times \tau_{\eta}$ for $\kappa \geq 1$, and the precision of every other trader's 
signal to be $\gamma \times \tau_{\eta}$ for $\gamma \in(0,1]$. The price (44) is an equilibrium price if and only if

$$
\begin{aligned}
A & =\frac{[(N-1) \gamma+\kappa] \tau_{\eta}}{N\left\{\tau_{\epsilon}+[(N-1) \gamma+\kappa] \tau_{\eta}+\tau_{\theta}\right\}}, \\
B & =\frac{\tau_{\theta}}{\tau_{\epsilon}+[(N-1) \gamma+\kappa] \tau_{\eta}+\tau_{\theta}} .
\end{aligned}
$$

The coefficient $A$ increases in $\kappa$ and $\gamma$. The coefficient $B$ decreases in $\kappa$ and $\gamma$.

Fixing the contemptuousness parameter $\gamma$, more overconfidence (larger $\kappa$ ) causes traders to attach larger weight to their own private signals and smaller weight to the public signal when forming conditional expectations. This causes $A$ to increase and $B$ to decrease. Fixing instead the overconfidence parameter $\kappa$, more contemptuousness (smaller $\gamma$ ) causes traders to attach smaller weight to other traders' private signals (as revealed by price) and larger weight to the public signal when forming conditional expectations. This causes $A$ to decrease and $B$ to increase. Contemptuousness thus moves $A$ and $B$ from their REE values in the same direction as cursedness does, while overconfidence moves them in the opposite direction.

Overconfidence and contemptuousness yield dramatically different predictions than cursedness about trading volume. Recall from Propositions 6 and 8 that under full or partial cursedness, per-trader volume grows as the market becomes large and hence aggregate volume converges to infinity. Under overconfidence or contemptuousness instead, per-trader volume converges to zero, and aggregate volume converges to a finite limit. Thus, overconfidence or contemptuousness on their own are not sufficient to generate volume in large markets, while cursedness is.

Proposition 16 Suppose that there are no endowment shocks and all traders have the same riskaversion coefficient $\alpha$ and private-signal precision $\tau_{\eta}$. Suppose also that each trader misperceives the precision of his private signal to be $\kappa \times \tau_{\eta}$ for $\kappa \geq 1$, and the precision of every other trader's signal to be $\gamma \times \tau_{\eta}$ for $\gamma \in(0,1]$. The expected volume that each trader generates is

$$
\frac{(\kappa-\gamma) \tau_{\zeta} \sqrt{2(N-1) \tau_{\eta}}}{\alpha\left[\tau_{\epsilon}+\tau_{\zeta}+[(N-1) \gamma+\kappa] \tau_{\eta}+\tau_{\theta}\right] \sqrt{\pi N}}
$$

Volume declines to zero as the number $N$ of traders grows large. Aggregate volume, summed across traders, converges to a finite limit. 
The intuition why per-trader volume converges to zero goes as follows. Overconfident or contemptuous traders trade for two reasons: first, each thinks that the price overweights all other traders' signals; second, each thinks that the price underweights his own signal. Since each trader inverts the price to perfectly infer the average signal of all other traders, the price would not change if all private signals were made public. If all signals were public, then each trader's expectation of the asset payoff $d$ would be a weighted average of his own signal, the average signal of other traders, and the public signal $s$. For any $\kappa \geq 1$ and $\gamma \in(0,1]$, as $N$ grows large, each trader puts arbitrarily more weight on the average signal of other traders than on his own signal. Hence, the difference between any two traders' expectations of $d$ converges to zero, and so does per-trader volume. ${ }^{10}$ Only when $\gamma=0$, which is observationally equivalent to full cursedness in our model, does per-trader volume not vanish in large markets.

Overconfident per-trader volume disappears in our model because each trader, no matter how overconfident, believes that the information conveyed by price about all other traders' signals trumps his own private signal in a sufficiently large market. This would not happen if traders believed that their private information conveyed something non-negligible beyond the information conveyed by a large-market price. Odean (1998) implicitly makes this assumption by assuming that the $N$ traders in the market observe $M<N$ signals in such a way that $\frac{N}{M}>1$ observe each signal. He computes volume when $\gamma=1$ and $N \rightarrow \infty$, but holding $M$ fixed. Whatever the value of $M$, each signal conveys non-negligible information beyond that conveyed by the set of other signals.

We conclude with the observation that overconfidence does have substantial effects when traders are cursed. Proposition 17 expresses volume when traders are overconfident and cursed. As when traders are cursed but not overconfident, per-trader volume grows as the market becomes large, and hence aggregate volume goes to infinity. Overconfidence produces an additional effect that increases volume even in the large-market limit. Yet it generates non-negligible per-trader volume only when traders are cursed, in which case it amplifies the effect of cursedness. In this sense, overconfidence

\footnotetext{
${ }^{10}$ The fact that equilibrium prices do not depend upon whether signals are public or private suggests that per-trader volume would be negligible in large markets even in complete-information models of overconfidence (e.g., Sheinkman and Xiong (2003)) if traders are risk averse.
} 
and cursedness work as complements. Intuitively, when traders fail to infer information from prices, overconfidence magnifies differences in their beliefs, producing even more trade. Although Proposition 17 assumes full cursedness, the same results hold under partial cursedness.

Proposition 17 Suppose that there are no endowment shocks and all traders have the same riskaversion coefficient $\alpha$ and private-signal precision $\tau_{\eta}$. Suppose also that all traders are fully cursed, and that each misperceives the precision of his private signal to be $\kappa \times \tau_{\eta}$ for $\kappa \geq 1$. The expected volume that each trader generates is

$$
\frac{\kappa \tau_{\zeta} \sqrt{2(N-1) \tau_{\eta}}}{\alpha\left(\tau_{\epsilon}+\tau_{\zeta}+\kappa \tau_{\eta}+\tau_{\theta}\right) \sqrt{\pi N}}
$$

Volume increases in the number of traders $N$ and in the overconfidence parameter $\kappa$.

\section{Discussion and Conclusion}

In this paper, we propose a market-equilibrium definition, cursed expectations equilibrium (CEE), for traders who fail to fully infer information from prices. We compare CEE to REE and find that although each cursed trader puts more weight on his private signal than a rational trader, because traders neglect that the price encodes other traders' information, CEE prices depend less on private signals and more on public signals than REE prices. Markets comprised entirely of cursed traders generate more trade than those comprised entirely of rationals; mixed markets can generate more trade still, as rationals employ momentum-trading strategies to take advantage of cursed traders. We contrast cursed trade to overconfidence- or contemptuousness-based trade, showing that cursed volume per trader grows with the size of the market, whereas per-trader volume under overconfidence or contemptuousness declines to zero. Cursedness, however, can enhance overconfidence- or contemptuousness-based trade.

How can we extend cursed-expectations equilibrium to dynamic settings? In a cursed equilibrium, agents understand the relationship between their opponents' actions across periods. Consequently, if the private signal that agent $i$ receives in period $t$ feeds into both $p_{t}$ and $p_{t+1}$, then cursed agents will forecast $p_{t+1}$ more accurately from $p_{t}$ than befits the motivation behind cursedness. Indeed, a shortcoming of cursed equilibrium emphasized by Eyster and Rabin (2005) in 
their conclusion is that it captures failures of contingent thinking about the relationship between private information and action, but not those about the relationship between action and action. Extending the logic of CEE to multiple periods probably requires relaxing the assumption that agents correctly perceive the correlation among actions. One approach might resemble that of Eyster and Piccione (2013), who model a dynamic market where agents trade based on potentially incomplete models of the relationship between next period's price and current, publicly available economic variables. Each trader uses a theory comprised of some subset of these variables and forecasts next period's expected price correctly conditional upon all included variables. One major conceptual difference of their approach from CEE is that it offers no guidance as to which theories traders are likely to employ. ${ }^{11}$ Nevertheless, it might be marriageable to CEE by having a cursed trader $i$ forecast the next-period price $p_{t+1}$ conditional upon the current price $p_{t}$, his current (accumulated) private signal, $s_{i, t}$, and the current (accumulated) public signal $s_{t}$, excluding all lagged prices, as $E\left[p_{t+1} \mid\left\{s_{i, t}, s_{t}\right\}\right]$. With only two periods, and $p_{2}=d$, this formulation delivers CEE in our model. With more periods, it preserves the feature of our model that in every period traders fully appreciate the relationship that next period's price has to their signals.

A dynamic model may also highlight the role of a second shortcoming in inference from market prices that might affect traders. Eyster and Rabin (2010) develop the concept of "best response trailing naive inference": to the extent that agents $d o$ infer some private information from earlier actions (or prices), they may neglect how these earlier actions embed informational inferences. Models uniting a tendency to underappreciate the information about others' beliefs contained in market prices, with a tendency to underappreciate how those beliefs reflect redundant information, may provide ways of understanding the co-existence of under-inference as emphasized in this paper with unwarranted swings in group beliefs that also appear to be a hallmark of financial beliefs. In a narrower sense, combining the two errors may help better understand price dynamics. In a

\footnotetext{
${ }^{11}$ Rabin (2013) advocates the methodological advantages of "portable extensions of existing models" such as cursed equilibrium. Just as CEE identifies directional departures from REE in the static model of this paper, a dynamic extension is likely to make directional predictions that are merely accommodated by the framework of Eyster and Piccione (2013).
} 
dynamic market, agents in period $t+2$ may fail to appreciate how those in period $t+1$ infer private information from prices in period $t$. Suppose that the price is high in both periods $t$ and $t+1$. If some agents in period $t+2$ neglect that some of the positive information contained in the period $t+1$ price is in fact information agents gleaned from the high period $t$ price, then they will overestimate the positive information in the high prices, and push the price up further. This additional type of error in inference may then produce medium-run over-reaction to private information.

In Section 6, we showed the necessity in some settings of cursedness to "enable" overconfidence to explain appreciable per-trader volume of trade. We conclude by speculating how cursedness may similarly enable the study of various other biases in asset markets. Researchers have recently proposed that a number of statistical errors may be relevant for financial decisions, including overinference from small samples (see Rabin (2002) and Rabin and Vayanos (2010)) and non-belief in the law of large numbers (see Benjamin, Rabin, and Raymond (2013)). Predicting the consequences of these and other biases for markets where traders extract information from prices requires additional assumptions about traders' theories of one another's errors. Yet relatively little is known about how people reason about others' errors. In its extreme, cursedness provides a simple assumption about what people think of others' errors: they don't think about them at all. If models of errors are instead closed by assuming that people do agree to disagree about the meaning of private signals, then, much like with overconfidence in Section 6, we suspect that the per-trader volume of trade will be small in information-rich settings where each trader values the sum total of others' private information far more heavily than his own private signal. Finally, whereas we have assumed throughout the paper that private signals convey true information about the value of the risky asset, it might instead be the case that traders share a common misperception of the meaningfulness of signals. Indeed, much of "private information", especially that held by unsophisticated investors, may in fact be irrelevant. Otherwise rational traders who agree to agree on the information content of such private signals would, of course, not generate high volume of trade. Asymmetries in beliefs created by these false private signals would, however, produce cursed trade. 


\section{References}

[1] Akerlof, G. 1970, "The Market for 'Lemons': Quality Uncertainty and the Market Mechanism", Quarterly Journal of Economics 84(3): 488-500.

[2] Banerjee, S. 2011, "Learning from Prices and the Dispersion in Beliefs", Review of Financial Studies 24(9): 3025-3068.

[3] Banerjee, S., Kaniel, R. and I. Kremer 2009. "Price Drift as an Outcome of Differences in Higher Order Beliefs", Review of Financial Studies 22(9): 3707-3734.

[4] Banerjee, S. and I. Kremer 2010, "Disagreement and Learning: Dynamic Patterns of Trade", Journal of Finance 65(4): 1269-1302.

[5] Benjamin, D., Rabin, M. and C. Raymond 2013, "A Model of Non-Belief in the Law of Large Numbers", Mimeo.

[6] Bernard, V. and J. Thomas 1989, "Post-Earnings-Announcement Drift: Delayed Price Response or Risk Premium?", Journal of Accounting Research 27(Suppl):1-36.

[7] Carrillo, J. and T. Palfrey 2011, "No Trade", Games and Economic Behavior 71(1): 66-87.

[8] Daniel, K., Hirshleifer, D. and A. Subrahmanyam 1998, "Investor Psychology and Security Market under- and Overreactions", Journal of Finance 53(6): 1839-1885.

[9] Daniel, K., Hirshleifer, D. and A. Subrahmanyam 2001, "Overconfidence, Arbitrage, and Equilibrium Asset Pricing", Journal of Finance 56(3): 921-965.

[10] DeLong, J., Shleifer, A., Summers, L. and R. Waldmann 1990, "Noise Trader Risk in Financial Markets", Journal of Political Economy 98: 703-738.

[11] Diamond D. and R. Verrecchia 1981, "Information Aggregation in a Noisy Rational Expectations Equilibrium", Journal of Financial Economics 9: 221-235.

[12] Esponda, I. and E. Vespa 2013, "Hypothetical Thinking and Information Extraction: Strategic Voting in the Laboratory", Mimeo, NYU Stern. 
[13] Eyster, E. and M. Piccione 2013, “An Approach to Asset-Pricing Under Incomplete and Diverse Perceptions", Econometrica 81(4): 1483-1506.

[14] Eyster, E. and M. Rabin 2005, "Cursed Equilibrium", Econometrica 73(5): 1623-1672.

[15] Eyster, E. and M. Rabin 2010, "Naïve Herding in Rich-Information Settings", American Economic Journal: Microeconomics 2: 221-43.

[16] French, K. 2008, "Presidential Address: The Cost of Active Investing", Journal of Finance 63(4): 1537-1573.

[17] Griffin, D. and A. Tversky 1992, "The Weighing of Evidence and the Determinants of Confidence", Cognitive Psychology 24(3): 411-435. r

[18] Grossman, S. 1976, "On the Efficiency of Competitive Stock Markets when Traders Have Diverse Information", Journal of Finance 31: 573-585.

[19] Harris, M. and A. Raviv 1993, "Difference of Opinion Make a Horse Race", Review of Financial Studies 6: 473-506.

[20] Harrison, J. M. and D. Kreps 1978, "Speculative Investor Behavior in a Stock Market with Heterogenous Expectations", Quarterly Journal of Economics 93(2): 323-336.

[21] Hellwig, M. 1980, "On the Aggregation of Information in Competitive Markets", Journal of Economic Theory 22: 477-498.

[22] Hirshleifer, J. 1971, "The Private and Social Value of Information and the Reward to Inventive Activity", American Economic Review 61(4): 561-574.

[23] Holt, C. and R. Sherman 1994, "The Loser's Curse", American Economic Review 84(3): 642652.

[24] Hong, H. J. Scheinkman and W. Xiong 2006. "Asset Float and Speculative Bubbles", Journal of Finance, American Finance Association 61: 1073-1117. 
[25] Hong, H. and J. Stein 1999, "A Unified Theory of Underreaction, Momentum Trading and Overreaction in Asset Markets", Journal of Finance 54(6): 2143-2184.

[26] Hong, H. and J. Stein 2007, "Disagreement and the Stock Market", Journal of Economic Perspectives 21(2): 109-128.

[27] Jegadeesh, N. and S. Titman 1993, "Returns to Buying Winners and Selling Losers: Implications for Stock Market Efficiency", Journal of Finance 48: 65-91.

[28] Jegadeesh, N. and S. Titman 2011, "Momentum", Annual Review of Financial Economics 3: 493-509.

[29] Kagel, J. and D. Levin 2002, Common Value Auctions and the Winner's Curse, Princeton University Press.

[30] Kandel, E. and N. Pearson 1995, "Differential Interpretation of Public Signals and Trade in Speculative Markets", Journal of Political Economy 103: 83172.

[31] Malmendier, U. and G. Tate 2008, "Who Makes Acquisitions? CEO Overconfidence and the Market's Reaction", Journal of Financial Economics 89(1): 20-43.

[32] Milgrom, P. and N. Stokey 1982, "Information, Trade and Common Knowledge", Journal of Economic Theory 26(1): 17-27.

[33] Morris, S. 1996, "Speculative Investor Behavior and Learning", Quarterly Journal of Economics 111: 1111-1133.

[34] Odean, T. 1998, "Volume, Volatility, and Price when all Traders Are Above Average", Journal of Finance 53(6): 1887-1934.

[35] Rabin, M. 2002, "Inference by Believers in the Law of Small Numbers", Quarterly Journal of Economics 117(3): 775-816.

[36] Rabin, M. 2013, "An Approach to Incorporating Psychology into Economics", American Economic Review (Papers \& Proceedings) 103(3): 617-22. 
[37] Rabin, M. and D. Vayanos 2010, "The Gambler's and Hot-Hand Fallacies: Theory and Applications", Review of Economic Studies 77: 730-778.

[38] Samuelson, W. and Bazerman, M. 1985, "The Winner's Curse in Bilateral Negotiations." In Research in Experimental Economics, vol. 3, Vernon L. Smith, ed., Greenwich, CT: JAI Press.

[39] Scheinkman, J. and W. Xiong 2003, "Overconfidence and Speculative Bubbles", Journal of Political Economy 111(6): 1183-1219.

[40] Varian, H. 1989, "Divergence of Opinion in Complete Markets: A Note", Journal of Finance 40: 309-317.

[41] Weizsäcker, G. 2010, "Do We Follow Others when We Should? A Simple Test of Rational Expectations", American Economic Review 100: 2340-2360. 


\section{APPENDIX}

\section{A Cursed Equilibrium}

Cursed equilibrium is defined in finite Bayesian games of the form

$$
\left(\left\{A_{i}\right\}_{i=1, \ldots, N},\left\{T_{i}\right\}_{i=0, \ldots, N}, p,\left\{u_{i}\right\}_{i=1, \ldots, N}\right) .
$$

For each player $i=1, \ldots, N, A_{i}$ is a finite set of available actions and $T_{i}$ is a finite set of types, including one, $T_{0}$, for nature. We denote the set of action profiles by $A \equiv \underset{i=1, \ldots, N}{\times} A_{i}$ and the set of type profiles by $T \equiv \underset{i=0, \ldots, N}{\times} T_{i}$. We assume that all players share the common prior probability distribution $p$ over $T$. Player $i$ 's utility function is $u_{i}: A \times T \rightarrow \mathbb{R}$.

A strategy for player $i, \sigma_{i}: T_{i} \rightarrow \triangle A_{i}$, specifies a probability distribution over actions for each type. We denote by $\sigma_{i}\left(a_{i} \mid t_{i}\right)$ the probability that type $t_{i}$ plays action $a_{i}$ when he follows strategy $\sigma_{i}$. We denote the set of action profiles for players other than $i$ by $A_{-i} \equiv \underset{j \neq 0, i}{\times} A_{j}$, and the set of type profiles for nature and players other than $i$ by $T_{-i} \equiv \underset{j \neq i}{\times} T_{j}$. We denote by $a_{-i}$ and $t_{-i}$ generic elements of these sets. We denote by $\sigma_{-i}\left(a_{-i} \mid t_{-i}\right)$ the probability that types $t_{-i}$ play action profile $a_{-i}$ when they follow strategy $\sigma_{-i} \equiv\left\{\sigma_{j}\right\}_{j \neq 0, i}$. Finally, we denote by $p\left(t_{-i} \mid t_{i}\right)$ the distribution of player $i$ 's beliefs about other players' types conditional on his own type $t_{i}$. The standard solution concept for these games is Bayesian Nash equilibrium.

Definition 2 A strategy profile $\sigma$ is a Bayesian Nash equilibrium if for each player $i$, each type $t_{i} \in T_{i}$, and each $a_{i}^{*}$ such that $\sigma_{i}\left(a_{i}^{*} \mid t_{i}\right)>0$ :

$$
a_{i}^{*} \in \arg \max _{a_{i} \in A_{i}} \sum_{t_{-i}} p\left(t_{-i} \mid t_{i}\right)\left(\sum_{a_{-i}} \sigma_{-i}\left(a_{-i} \mid t_{-i}\right) u_{i}\left(a_{i}, a_{-i} ; t_{i}, t_{-i}\right)\right) .
$$

To define cursed equilibrium, we compute for each type of each player the average strategy of other players, averaged over the other players' types. For type $t_{i}$ of player $i$ we define

$$
\bar{\sigma}_{-i}\left(a_{-i} \mid t_{i}\right) \equiv \sum_{t_{-i} \in T_{-i}} p_{i}\left(t_{-i} \mid t_{i}\right) \cdot \sigma_{-i}\left(a_{-i} \mid t_{-i}\right) .
$$


This is the marginal probability that other players play action profile $a_{-i}$, and is derived by averaging over type profiles $t_{-i}$ the probabilities $\sigma_{-i}\left(a_{-i} \mid t_{-i}\right)$ that other players play $a_{-i}$ conditional on $t_{-i}$. We associate to each player $i$ a cursedness parameter $\chi_{i} \in[0,1]$.

Definition $3 A$ strategy profile $\sigma$ is a cursed equilibrium if for each player $i$, each type $t_{i} \in T_{i}$, and each $a_{i}^{*}$ such that $\sigma_{i}\left(a_{i}^{*} \mid t_{i}\right)>0$ :

$$
\begin{array}{r}
a_{i}^{*} \in \arg \max _{a_{i} \in A_{i}} \sum_{t_{-i}} p\left(t_{-i} \mid t_{i}\right)\left(\sum_{a_{-i}}\left(1-\chi_{i}\right) \sigma_{-i}\left(a_{-i} \mid t_{-i}\right) u_{i}\left(a_{i}, a_{-i} ; t_{i}, t_{-i}\right)\right. \\
\left.+\chi_{i} \bar{\sigma}_{-i}\left(a_{-i} \mid t_{i}\right) u_{i}\left(a_{i}, a_{-i} ; t_{i}, t_{-i}\right)\right) .
\end{array}
$$

Player $i$ best-responds to beliefs that with probability $1-\chi_{i}$ the other players' actions depend on their types (the probability of action profile $a_{-i}$ in (A.2) is conditional on type profile $t_{-i}$ ) and with probability $\chi_{i}$ actions do not depend on types (the probability of $a_{-i}$ in (A.2) is the marginal). When $\chi_{i}=0$, player $i$ is rational, and his objective is as in Bayesian Nash equilibrium (Eq. (A.1)). When $\chi_{i}=1$, player $i$ is fully cursed, and neglects entirely the relationship between the other players' actions and their types. Note that while cursed players fail to map actions to types, they assess correctly the probability distribution of other players' actions.

Applying the definition (A.2) to the trading game considered in Section 2, we find that the buyer's objective is $F(p) \times((1-\chi) \mathrm{E}[s \mid s \leq p]+\chi \mathrm{E}[s]-p)$ : the buyer believes that with probability $1-\chi$ the seller's decision to sell conveys information about the asset, and with probability $\chi$ it does not.

\section{B Proofs}

We first prove the following lemma, which we use for proving Proposition 1.

Lemma A.1 Suppose that the variables $\left(x,\left\{y_{i}\right\}_{i=1, . ., K}\right)$ are normal, independent, with mean zero and precisions $\left(\tau_{x},\left\{\tau_{y_{i}}\right\}_{i=1, . ., K}\right)$. Then, the distribution of $x$ conditional on $\left\{x+y_{i}\right\}_{i=1, . ., K}$ is normal 
with mean

$$
\mathrm{E}\left(x \mid\left\{x+y_{i}\right\}_{i=1, . ., K}\right)=\sum_{i=1}^{K} \frac{\tau_{y_{i}}}{\tau_{x}+\sum_{j=1}^{K} \tau_{y_{j}}}\left(x+y_{i}\right)
$$

and precision

$$
\tau\left(x \mid\left\{x+y_{i}\right\}_{i=1, . ., K}\right)=\tau_{x}+\sum_{i=1}^{K} \tau_{y_{i}} .
$$

Proof. The conditional mean and variance can be computed from the regression

$$
x=\sum_{i=1}^{K} \beta_{i}\left(x+y_{i}\right)+e,
$$

where $\left\{\beta_{i}\right\}_{i=1, . ., K}$ are the regression coefficients and $e$ is the error term. Taking covariances of both sides with $x+y_{i}$ and noting that $\left(x,\left\{y_{i}\right\}_{i=1, . ., K}, e\right)$ are independent, we find

$$
\begin{aligned}
& \operatorname{Cov}\left(x, x+y_{i}\right)=\sum_{j=1}^{K} \beta_{j} \operatorname{Cov}\left(x+y_{j}, x+y_{i}\right) \\
& \Rightarrow \frac{1}{\tau_{x}}=\beta_{i}\left(\frac{1}{\tau_{x}}+\frac{1}{\tau_{y_{i}}}\right)+\sum_{j \neq i} \beta_{j} \frac{1}{\tau_{x}} \\
& \Rightarrow \beta_{i}=\frac{\tau_{y_{i}}}{\tau_{x}}\left(1-\sum_{j=1}^{K} \beta_{j}\right) .
\end{aligned}
$$

Summing (A.3) across $i$ and solving for $\sum_{j=1}^{K} \beta_{j}$, we find

$$
\sum_{j=1}^{K} \beta_{j}=\frac{\sum_{j=1}^{K} \tau_{y_{j}}}{\tau_{x}+\sum_{j=1}^{K} \tau_{y_{j}}} .
$$

Substituting $\sum_{j=1}^{K} \beta_{j}$ from (A.4) into (A.3), we find

$$
\beta_{i}=\frac{\tau_{y_{i}}}{\tau_{x}+\sum_{j=1}^{K} \tau_{y_{j}}} .
$$

Since

$$
\mathrm{E}\left(x \mid\left\{x+y_{i}\right\}_{i=1, . ., K}\right)=\sum_{i=1}^{K} \beta_{i}\left(x+y_{i}\right),
$$


(A.5) implies (A.1). Taking variances of both sides and noting that $\left(x,\left\{y_{i}\right\}_{i=1, . ., K}, e\right)$ are independent, we find

$$
\begin{aligned}
& \operatorname{Var}(x)=\left(\sum_{i=1}^{K} \beta_{i}\right)^{2} \operatorname{Var}(x)+\sum_{i=1}^{K} \beta_{j}^{2} \operatorname{Var}\left(y_{i}\right)+\operatorname{Var}(e) \\
& \Rightarrow \frac{1}{\tau_{e}}=\frac{1}{\tau_{x}}\left[1-\left(\frac{\sum_{i=1}^{K} \tau_{y_{i}}}{\tau_{x}+\sum_{i=1}^{K} \tau_{y_{i}}}\right)^{2}\right]-\sum_{i=1}^{K} \frac{1}{\tau_{y_{i}}}\left(\frac{\tau_{y_{i}}}{\tau_{x}+\sum_{j=1}^{K} \tau_{y_{j}}}\right)^{2} \\
& \Rightarrow \frac{1}{\tau_{e}}=\frac{1}{\tau_{x}+\sum_{i=1}^{K} \tau_{y_{i}}}
\end{aligned}
$$

where the second step follows from (A.4) and (A.5). Since

$$
\tau\left(x \mid\left\{x+y_{i}\right\}_{i=1, . ., K}\right)=\tau_{e},
$$

(A.6) implies (A.2).

Proof of Proposition 1. We first determine traders' demand functions using (8). Since $d=$ $\bar{d}+\epsilon+\zeta$ and $\zeta$ is independent of traders' information $I_{i}$,

$$
\begin{aligned}
& \mathrm{E}\left(d \mid I_{i}\right)=\bar{d}+E\left(\epsilon \mid I_{i}\right), \\
& \operatorname{Var}\left(d \mid I_{i}\right)=\operatorname{Var}\left(\epsilon \mid I_{i}\right)+\operatorname{Var}(\zeta)=\frac{1}{\tau\left(\epsilon \mid I_{i}\right)}+\frac{1}{\tau_{\zeta}} .
\end{aligned}
$$

Using Lemma A.1 with $x=\epsilon, K=3$ and $\left\{y_{j}\right\}_{j=1,2,3}=\left(\eta_{i}, \eta, \xi_{i}\right)$, we find

$$
\begin{aligned}
& \mathrm{E}\left(d \mid I_{i r}\right)=\bar{d}+\frac{\tau_{\eta_{i}}}{\tau_{\epsilon}+\tau_{\eta_{i}}+\tau_{\theta}+\tau_{\xi_{i}}} s_{i}+\frac{\tau_{\theta}}{\tau_{\epsilon}+\tau_{\eta_{i}}+\tau_{\theta}+\tau_{\xi_{i}}} s+\frac{\tau_{\xi_{i}}}{\tau_{\epsilon}+\tau_{\eta_{i}}+\tau_{\theta}+\tau_{\xi_{i}}}\left(\epsilon+\xi_{i}\right), \\
& \operatorname{Var}\left(d \mid I_{i r}\right)=\frac{1}{\tau_{\epsilon}+\tau_{\eta_{i}}+\tau_{\theta}+\tau_{\xi_{i}}}+\frac{1}{\tau_{\zeta}}
\end{aligned}
$$

Using Lemma A.1 with $x=\epsilon, K=2$ and $\left\{y_{j}\right\}_{j=1,2}=\left(\eta_{i}, \eta\right)$, we find

$$
\begin{aligned}
& \mathrm{E}\left(d \mid I_{i c}\right)=\bar{d}+\frac{\tau_{\eta_{i}}}{\tau_{\epsilon}+\tau_{\eta_{i}}+\tau_{\theta}} s_{i}+\frac{\tau_{\theta}}{\tau_{\epsilon}+\tau_{\eta_{i}}+\tau_{\theta}} s, \\
& \operatorname{Var}\left(d \mid I_{i c}\right)=\frac{1}{\tau_{\epsilon}+\tau_{\eta_{i}}+\tau_{\theta}}+\frac{1}{\tau_{\zeta}} .
\end{aligned}
$$

Substituting (A.9), (A.10), (A.11) and (A.12) into (8), we can write the demand of trader $i$ as

$$
x_{i}=\frac{\bar{d}+\left(1-\chi_{i}\right) \frac{\tau_{\eta_{i}} s_{i}+\tau_{\theta} s+\tau_{\xi_{i}}\left(\epsilon+\xi_{i}\right)}{\tau_{\epsilon}+\tau_{\eta_{i}}+\tau_{\theta}+\tau_{\xi_{i}}}+\chi_{i} \frac{\tau_{\eta_{i}} s_{i}+\tau_{\theta} s}{\tau_{\epsilon}+\tau_{\eta_{i}}+\tau_{\theta}}-p}{\alpha_{i}\left[\left(1-\chi_{i}\right) \frac{1}{\tau_{\epsilon}+\tau_{\eta_{i}}+\tau_{\theta}+\tau_{\xi_{i}}}+\chi_{i} \frac{1}{\tau_{\epsilon}+\tau_{\eta_{i}}+\tau_{\theta}}+\frac{1}{\tau_{\zeta}}\right]}-z_{i} .
$$


We next substitute (A.13) into the market-clearing condition (5), use (6) to write $p$ in terms of $\left(\left\{s_{i}\right\}_{i=1, . ., N}, s,\left\{z_{i}\right\}_{i=1, . ., N}\right)$, and use (9) to write $\epsilon+\xi_{i}$ in terms of $\left(\left\{s_{i}\right\}_{j \neq i},\left\{z_{i}\right\}_{j \neq i}\right)$. This yields an equation that is linear in $\left(\left\{s_{i}\right\}_{i=1, . ., N}, s,\left\{z_{i}\right\}_{i=1, . ., N}\right)$. Identifying terms in $s_{i}$ yields (11). Identifying terms of $s$ yields (12). Identifying terms in $z_{i}$ yields

$$
\begin{aligned}
& 1-\frac{\left(1-\chi_{i}\right) \tau_{\zeta} \tau_{\xi_{i}} \frac{C_{i}}{\sum_{k \neq i} A_{k}}\left(\tau_{\epsilon}+\tau_{\eta_{i}}+\tau_{\theta}\right)}{\alpha_{i}\left[\left(\tau_{\epsilon}+\tau_{\eta_{i}}+\tau_{\theta}\right)\left(\tau_{\epsilon}+\tau_{\eta_{i}}+\tau_{\theta}+\tau_{\xi_{i}}\right)+\tau_{\zeta}\left(\tau_{\epsilon}+\tau_{\eta_{i}}+\tau_{\theta}+\chi_{i} \tau_{\xi_{i}}\right)\right]} \\
& =C_{i} \tau_{\zeta} \sum_{j=1}^{N} \frac{\left(\tau_{\epsilon}+\tau_{\eta_{j}}+\tau_{\theta}\right)\left(\tau_{\epsilon}+\tau_{\eta_{j}}+\tau_{\theta}+\tau_{\xi_{j}}\right)-\left(1-\chi_{j}\right) \tau_{\xi_{j}} \frac{1}{\sum_{k \neq j} A_{k}}\left(\tau_{\epsilon}+\tau_{\eta_{j}}+\tau_{\theta}\right)}{\alpha_{j}\left[\left(\tau_{\epsilon}+\tau_{\eta_{j}}+\tau_{\theta}\right)\left(\tau_{\epsilon}+\tau_{\eta_{j}}+\tau_{\theta}+\tau_{\xi_{j}}\right)+\tau_{\zeta}\left(\tau_{\epsilon}+\tau_{\eta_{j}}+\tau_{\theta}+\chi_{j} \tau_{\xi_{j}}\right)\right]} .
\end{aligned}
$$

Combining (A.14) with (11) yields (13).

Proof of Proposition 2. Setting $\chi_{i}=0$ for all $i$, we can simplify (11) to

$$
\frac{\tau_{\eta_{i}}-\tau_{\xi_{i}} \frac{A_{i}}{\sum_{k \neq i} A_{k}}}{\alpha_{i}\left(\tau_{\epsilon}+\tau_{\zeta}+\tau_{\eta_{i}}+\tau_{\theta}+\tau_{\xi_{i}}\right)}=A_{i} \sum_{j=1}^{N} \frac{\tau_{\epsilon}+\tau_{\eta_{j}}+\tau_{\theta}+\tau_{\xi_{j}}-\tau_{\xi_{j}} \frac{1}{\sum_{k \neq j} A_{k}}}{\alpha_{j}\left(\tau_{\epsilon}+\tau_{\zeta}+\tau_{\eta_{j}}+\tau_{\theta}+\tau_{\xi_{j}}\right)} \equiv A_{i} \mathcal{Z} .
$$

Since $\left\{\sigma_{z_{i}}\right\}_{i=1, \ldots, N}=0,(10)$ implies that

$$
\tau_{\xi_{i}}=\frac{\left(\sum_{j \neq i} A_{j}\right)^{2}}{\sum_{j \neq i} \frac{A_{j}^{2}}{\tau_{\eta_{j}}}}
$$

Substituting $\tau_{\xi_{i}}$ from (A.16) into the left-hand side of (A.15), we can write (A.15) as

$$
\begin{aligned}
& \frac{\tau_{\eta_{i}}}{\alpha_{i}\left(\tau_{\epsilon}+\tau_{\zeta}+\tau_{\eta_{i}}+\tau_{\theta}+\tau_{\xi_{i}}\right) \sum_{j \neq i} \frac{A_{j}^{2}}{\tau_{\eta_{j}}}}\left(\sum_{j \neq i} \frac{A_{j}^{2}}{\tau_{\eta_{j}}}-\frac{A_{i}}{\tau_{\eta_{i}}} \sum_{j \neq i} A_{j}\right)=A_{i} \mathcal{Z} \\
& \Rightarrow \sum_{j=1}^{N} \frac{A_{j}^{2}}{\tau_{\eta_{j}}}-\frac{A_{i}}{\tau_{\eta_{i}}} \sum_{j=1}^{N} A_{j}=\frac{\alpha_{i}\left(\tau_{\epsilon}+\tau_{\zeta}+\tau_{\eta_{i}}+\tau_{\theta}+\tau_{\xi_{i}}\right) A_{i} \sum_{j \neq i} \frac{A_{j}^{2}}{\tau_{\eta_{j}}}}{\tau_{\eta_{i}}} \mathcal{Z} .
\end{aligned}
$$

Multiplying (A.17) by $A_{i}$ and summing over $i$, we find

$$
0=\sum_{i=1}^{N} A_{i} \sum_{i=1}^{N} \frac{A_{i}^{2}}{\tau_{\eta_{i}}}-\sum_{i=1}^{N} A_{i} \sum_{i=1}^{N} \frac{A_{i}^{2}}{\tau_{\eta_{i}}}=\sum_{i=1}^{N}\left[\frac{\alpha_{i}\left(\tau_{\epsilon}+\tau_{\zeta}+\tau_{\eta_{i}}+\tau_{\theta}+\tau_{\xi_{i}}\right) A_{i}^{2} \sum_{j \neq i} \frac{A_{j}^{2}}{\tau_{\eta_{j}}}}{\tau_{\eta_{i}}}\right] \mathcal{Z},
$$

which implies that $\mathcal{Z}=0$. Eq. (A.17) then implies that

$$
\sum_{j=1}^{N} \frac{A_{j}^{2}}{\tau_{\eta_{j}}}-\frac{A_{i}}{\tau_{\eta_{i}}} \sum_{j=1}^{N} A_{j}=0
$$


which in turn implies that

$$
A_{i}=\lambda \tau_{\eta_{i}}
$$

for a constant $\lambda$ that does not depend on $i$. Substituting $A_{i}$ from (A.18) into (A.16), we find

$$
\tau_{\xi_{i}}=\sum_{j \neq i} \tau_{\eta_{j}}
$$

Substituting $\left(A_{i}, \tau_{\xi_{i}}\right)$ from (A.18) and (A.19) into the definition of $\mathcal{Z}$ in (15), and recalling that $\mathcal{Z}=0$, we find

$$
\begin{aligned}
& \left(\sum_{i=1}^{N} \frac{1}{\alpha_{i}}\right) \frac{\tau_{\epsilon}+\sum_{i=1}^{N} \tau_{\eta_{i}}+\tau_{\theta}}{\tau_{\epsilon}+\tau_{\zeta}+\sum_{i=1}^{N} \tau_{\eta_{i}}+\tau_{\theta}}-\frac{1}{\lambda}\left(\sum_{i=1}^{N} \frac{1}{\alpha_{i}}\right) \frac{1}{\tau_{\epsilon}+\tau_{\zeta}+\sum_{i=1}^{N} \tau_{\eta_{i}}+\tau_{\theta}}=0 \\
& \Rightarrow \lambda=\frac{1}{\tau_{\epsilon}+\sum_{i=1}^{N} \tau_{\eta_{i}}+\tau_{\theta}} .
\end{aligned}
$$

Eqs. (A.18) and (A.20) imply (15). Eq. (16) follows similarly by substituting $\tau_{\xi_{i}}$ from (A.19) into (12). Substituting $\left(\epsilon+\xi_{i}, p,\left\{A_{j}\right\}_{j=1, . ., N}, B, \tau_{\xi_{i}}\right)$ from (9), (14), (15), (16) and (A.19) into (A.13) for $\chi_{i}=0$, we find that the numerator in (A.13) is zero. Since, in addition $z_{i}=0$, trader $i$ 's demand is zero for the equilibrium price. Therefore, there is no trade.

Proof of Proposition 3. Setting $\chi_{i}=1$ for all $i$, we can simplify (11) to

$$
\frac{\tau_{\eta_{i}}}{\alpha_{i}\left(\tau_{\epsilon}+\tau_{\zeta}+\tau_{\eta_{i}}+\tau_{\theta}\right)}=A_{i} \sum_{j=1}^{N} \frac{\tau_{\epsilon}+\tau_{\eta_{j}}+\tau_{\theta}}{\alpha_{j}\left(\tau_{\epsilon}+\tau_{\zeta}+\tau_{\eta_{j}}+\tau_{\theta}\right)} .
$$

Solving for $A_{i}$ using (A.21) yields (17). Eq. (18) follows similarly from (12).

Proof of Proposition 4. Eqs. (16) and (18) imply that (i) holds if

$$
\tau_{\epsilon}+\sum_{i=1}^{N} \tau_{\eta_{i}}+\tau_{\eta}>\frac{\sum_{i=1}^{N} \frac{\tau_{\epsilon}+\tau_{\eta_{i}}+\tau_{\theta}}{\alpha_{i}\left(\tau_{\epsilon}+\tau_{\zeta}+\tau_{\eta_{i}}+\tau_{\theta}\right)}}{\sum_{i=1}^{N} \frac{1}{\alpha_{i}\left(\tau_{\epsilon}+\tau_{\zeta}+\tau_{\eta_{i}}+\tau_{\theta}\right)}}=\sum_{i=1}^{N} w_{i}\left(\tau_{\epsilon}+\tau_{\eta_{i}}+\tau_{\theta}\right),
$$

where

$$
w_{i} \equiv \frac{\frac{1}{\alpha_{i}\left(\tau_{\epsilon}+\tau_{\zeta}+\tau_{\eta_{i}}+\tau_{\theta}\right)}}{\sum_{i=1}^{N} \frac{1}{\alpha_{i}\left(\tau_{\epsilon}+\tau_{\zeta}+\tau_{\eta_{i}}+\tau_{\theta}\right)}} .
$$


Since the weights $\left\{w_{i}\right\}_{i=1, . ., N}$ are positive and sum to one, the right-hand side of (A.22) is smaller than

$$
\tau_{\epsilon}+\max _{i=1, . ., N} \tau_{\eta_{i}}+\tau_{\theta}<\tau_{\epsilon}+\sum_{i=1}^{N} \tau_{\eta_{i}}+\tau_{\theta},
$$

and so (A.22) holds.

Eqs. (15) and (17) imply that the first statement in (ii) holds if

$$
\frac{\tau_{\theta}+\sum_{i=1}^{N} \tau_{\eta_{i}}+\tau_{\theta}}{\sum_{i=1}^{N} \tau_{\eta_{i}}}<\frac{\sum_{i=1}^{N} \frac{\tau_{\epsilon}+\tau_{\eta_{i}}+\tau_{\theta}}{\alpha_{i}\left(\tau_{\epsilon}+\tau_{\zeta}+\tau_{\eta_{i}}+\tau_{\theta}\right)}}{\sum_{i=1}^{N} \frac{\tau_{\eta_{i}}}{\alpha_{i}\left(\tau_{\epsilon}+\tau_{\zeta}+\tau_{\eta_{i}}+\tau_{\theta}\right)}}=\sum_{i=1}^{N} w_{i} \frac{\tau_{\epsilon}+\tau_{\eta_{i}}+\tau_{\theta}}{\tau_{\eta_{i}}},
$$

where

$$
w_{i} \equiv \frac{\frac{\tau_{\eta_{i}}}{\alpha_{i}\left(\tau_{\epsilon}+\tau_{\zeta}+\tau_{\eta_{i}}+\tau_{\theta}\right)}}{\sum_{i=1}^{N} \frac{\tau_{\eta_{i}}}{\alpha_{i}\left(\tau_{\epsilon}+\tau_{\zeta}+\tau_{\eta_{i}}+\tau_{\theta}\right)}} .
$$

Since the weights $\left\{w_{i}\right\}_{i=1, . ., N}$ are positive and sum to one, the right-hand side of (A.22) is larger than

$$
\min _{i=1, . ., N} \frac{\tau_{\epsilon}+\tau_{\eta_{i}}+\tau_{\theta}}{\tau_{\eta_{i}}}>\frac{\tau_{\theta}+\sum_{i=1}^{N} \tau_{\eta_{i}}+\tau_{\theta}}{\sum_{i=1}^{N} \tau_{\eta_{i}}},
$$

and so (A.23) holds. When traders are symmetric, the first statement in (ii) implies that $A_{i}$ is smaller in the fully cursed than in the rational case for all $i$. To show the second statement in (ii), it suffices to show an example where $A_{i}$ is larger in the fully cursed than in the rational case for some $i$. Suppose that trader $i$ is much less risk averse than the other traders. Eq. (17) then implies that

$$
A_{i} \approx \frac{\tau_{\eta_{i}}}{\tau_{\epsilon}+\tau_{\eta_{i}}+\tau_{\theta}}
$$

in the fully cursed case. This is larger than $A_{i}$ in the rational case, given by (15).

Eqs. (15), (16), (17) and (18) imply that (iii) holds if

$$
\frac{\tau_{\eta_{i}}}{\tau_{\theta}} \frac{\frac{1}{\alpha_{i}\left(\tau_{\epsilon}+\tau_{\zeta}+\tau_{\eta_{i}}+\tau_{\theta}\right)}}{\sum_{i=1}^{N} \frac{1}{\alpha_{i}\left(\tau_{\epsilon}+\tau_{\zeta}+\tau_{\eta_{i}}+\tau_{\theta}\right)}}<\frac{\tau_{\eta_{i}}}{\tau_{\theta}}
$$

which obviously holds. 
Proof of Proposition 5. The results for the rational case follow because Proposition 2 implies that

$$
p=\mathrm{E}\left(d \mid\left\{s_{i}\right\}_{i=1, . ., N}, s\right)
$$

which in turn implies that

$$
\mathrm{E}(d-p \mid I)=\mathrm{E}\left[d-\mathrm{E}\left(d \mid\left\{s_{i}\right\}_{i=1, . ., N}, s\right) \mid I\right]=\mathrm{E}(d \mid I)-\mathrm{E}(d \mid I)=0
$$

for any information set $I$ consisting of information revealed in Period 1. Since the expectation of $d-p$ conditional on $I$ is zero, the coefficients $\left(\gamma_{1}, \gamma_{2}, \gamma\right)$ in the regressions (20), (21) and (22) are zero.

We next turn to the fully cursed case. To show the result for regression (21), we follow the argument sketched after the proposition. Taking expectations of both sides of (19) for $\chi_{i}=1$ for all $i$, and noting that $\operatorname{Var}\left(d \mid I_{i c}\right)$ is a constant, we find

$$
\mathrm{E}(p \mid s)=\frac{\sum_{i=1}^{N} \frac{\mathrm{E}\left[\mathrm{E}\left(d \mid I_{i c}\right) \mid s\right]}{\alpha_{i} \operatorname{Var}\left(d \mid I_{i c}\right)}}{\sum_{i=1}^{N} \frac{1}{\alpha_{i} \operatorname{Var}\left(d \mid I_{i c}\right)}}=\frac{\sum_{i=1}^{N} \frac{\mathrm{E}(d \mid s)}{\alpha_{i} \operatorname{Var}\left(d \mid I_{i c}\right)}}{\sum_{i=1}^{N} \frac{1}{\alpha_{i} \operatorname{Var}\left(d \mid I_{i c}\right)}}=E(d \mid s),
$$

where the second step follows from the law of iterative expectations because $I_{i c}$ includes $s$. Eq. (A.25) implies that

$$
\mathrm{E}(d-p \mid s)=\mathrm{E}(d \mid s)-\mathrm{E}(p \mid s)=0 .
$$

The coefficient $\gamma$ in the regression (21) is proportional to

$$
\operatorname{Cov}(d-p, s)=\mathrm{E}((d-p) s)-\mathrm{E}(d-p) \mathrm{E}(s)=\mathrm{E}(\mathrm{E}(d-p \mid s) s)-\mathrm{E}(\mathrm{E}(d-p \mid s)) \mathrm{E}(s)=0,
$$

where the second step follows from the law of iterative expectations and last step follows from (A.26).

To show the result for regression (22), we note that $\gamma$ is proportional to

$$
\operatorname{Cov}(d-p, p-\bar{d})=\left(1-\sum_{i=1}^{N} A_{i}-B\right)\left(\sum_{i=1}^{N} A_{i}+B\right) \sigma_{\epsilon}^{2}-\sum_{i=1}^{N} A_{i}^{2} \sigma_{\eta_{i}}^{2}-B^{2} \sigma_{\theta}^{2},
$$


where the equality follows from (1), (2) and (14). Substituting $\left(\left\{A_{i}\right\}_{i=1, . ., N}, B\right)$ from Proposition 3 , we find that $\operatorname{Cov}(d-p, p-\bar{d})$ has the same sign as

$$
\begin{aligned}
& \sum_{i=1}^{N} \frac{\tau_{\epsilon}}{\alpha_{i}\left(\tau_{\epsilon}+\tau_{\zeta}+\tau_{\eta_{i}}+\tau_{\theta}\right)} \sum_{i=1}^{N} \frac{\tau_{\eta_{i}}+\tau_{\theta}}{\alpha_{i}\left(\tau_{\epsilon}+\tau_{\zeta}+\tau_{\eta_{i}}+\tau_{\theta}\right)} \sigma_{\epsilon}^{2}-\sum_{i=1}^{N} \frac{\tau_{\eta_{i}}^{2} \sigma_{\eta_{i}}^{2}+\tau_{\theta}^{2} \sigma_{\theta}^{2}}{\alpha_{i}^{2}\left(\tau_{\epsilon}+\tau_{\zeta}+\tau_{\eta_{i}}+\tau_{\theta}\right)^{2}} \\
& =\sum_{i=1}^{N} \frac{1}{\alpha_{i}\left(\tau_{\epsilon}+\tau_{\zeta}+\tau_{\eta_{i}}+\tau_{\theta}\right)} \sum_{i=1}^{N} \frac{\tau_{\eta_{i}}+\tau_{\theta}}{\alpha_{i}\left(\tau_{\epsilon}+\tau_{\zeta}+\tau_{\eta_{i}}+\tau_{\theta}\right)}-\sum_{i=1}^{N} \frac{\tau_{\eta_{i}}+\tau_{\theta}}{\alpha_{i}^{2}\left(\tau_{\epsilon}+\tau_{\zeta}+\tau_{\eta_{i}}+\tau_{\theta}\right)^{2}},
\end{aligned}
$$

which is positive.

To show the result for regression (20), we compute $\left(\gamma_{1}, \gamma_{2}\right)$. Taking covariances of both sides of (20) with $p-\bar{d}$ and with $s$, yields respectively

$$
\begin{aligned}
& \operatorname{Cov}(d-p, p-\bar{d})=\gamma_{1} \operatorname{Var}(p-\bar{d})+\gamma_{2} \operatorname{Cov}(s, p-\bar{d}), \\
& \operatorname{Cov}(d-p, s)=\gamma_{1} \operatorname{Cov}(p-\bar{d}, s)+\gamma_{2} \operatorname{Var}(s) .
\end{aligned}
$$

Eqs. (A.27) and (A.28) form a linear system in $\left(\gamma_{1}, \gamma_{2}\right)$. Its solution is

$$
\begin{aligned}
\gamma_{1} & =\frac{\operatorname{Cov}(d-p, p-\bar{d}) \operatorname{Var}(s)-\operatorname{Cov}(d-p, s) \operatorname{Cov}(s, p-\bar{d})}{\operatorname{Var}(p-\bar{d}) \operatorname{Var}(s)-\operatorname{Cov}(s, p-\bar{d})^{2}} \\
\gamma_{2} & =-\frac{\operatorname{Cov}(d-p, p-\bar{d}) \operatorname{Cov}(p-\bar{d}, s)-\operatorname{Cov}(d-p, s) \operatorname{Var}(p-\bar{d})}{\operatorname{Var}(p-\bar{d}) \operatorname{Var}(s)-\operatorname{Cov}(s, p-\bar{d})^{2}} .
\end{aligned}
$$

Since $\operatorname{Cov}(d-p, s)=0$ and $V(s)>0,\left(\right.$ A.29) implies that $\gamma_{1}>0$. Since $\operatorname{Cov}(d-p, s)=0$ and $\operatorname{Cov}(p-\bar{d}, s)>0,\left(\right.$ A.30) implies that $\gamma_{2}>0$.

Proof of Corollary 1. The variance of the return $p-\bar{d}$ between Periods 0 and 1 is equal to the variance of $p$. The comparison between $\operatorname{Var}(p)$ in the rational and fully cursed cases will follow from the identities

$\operatorname{Var}\left[\mathrm{E}\left(d \mid\left\{s_{i}\right\}_{i=1, . ., N}, s\right)\right]=\operatorname{Var}\left[\mathrm{E}\left(d \mid\left\{s_{i}\right\}_{i=1, . ., N}, s\right)-p\right]+2 \operatorname{Cov}\left[\mathrm{E}\left(d \mid\left\{s_{i}\right\}_{i=1, . ., N}, s\right)-p, p\right]+\operatorname{Var}(p)$ 
and

$$
\begin{aligned}
\operatorname{Cov}\left[\mathrm{E}\left(d \mid\left\{s_{i}\right\}_{i=1, . ., N}, s\right)-p, p\right] & =\mathrm{E}\left[\left(\mathrm{E}\left(d \mid\left\{s_{i}\right\}_{i=1, \ldots, N}, s\right)-p\right) p\right]-\mathrm{E}\left[\mathrm{E}\left(d \mid\left\{s_{i}\right\}_{i=1, \ldots, N}, s\right)-p\right] E(p) \\
& =\mathrm{E}[(\mathrm{E}(d \mid p)-p) p]-[\mathrm{E}(d)-\mathrm{E}(p)] E(p) \\
& =\mathrm{E}[\gamma(p-\bar{d}) p] \\
& =\gamma \operatorname{Var}(p)
\end{aligned}
$$

where the second step in (A.32) follows from applying the law of iterative expectations, and the third step follows from $(22)$ and $E(d)=E(p)=\bar{d}$. When traders are fully cursed, (A.32) and Proposition 5 imply that the second term in the right-hand side of (A.31) is positive. Since the first term is also positive, (A.31) implies that

$$
\operatorname{Var}\left[\mathrm{E}\left(d \mid\left\{s_{i}\right\}_{i=1, \ldots, N}, s\right)\right]>\operatorname{Var}(p) .
$$

When traders are rational, (A.33) holds as an equality because of (A.24). Therefore, $\operatorname{Var}(p)$ is smaller when traders are fully cursed.

The comparison between $\operatorname{Var}(p)+\operatorname{Var}(d-p)$ in the rational and fully cursed cases will follow similarly from the identities

$$
\operatorname{Var}(d)=\operatorname{Var}(d-p)+2 \operatorname{Cov}(d-p, p)+\operatorname{Var}(p)
$$

and

$$
\operatorname{Cov}(d-p, p)=\mathrm{E}[\gamma(p-\bar{d}) p]=\gamma \operatorname{Var}(p)
$$

where the first step in (A.35) follows from (22). Eq. (A.35) and Proposition 5 imply that the second term in the right-hand side of (A.34) is positive when traders are fully cursed and zero when they are rational. Since the left-hand side of (A.34) is the same when traders are fully cursed and when they are rational, $\operatorname{Var}(p)+\operatorname{Var}(d-p)$ is smaller when they are fully cursed.

Proof of Proposition 6. Substituting $p$ from (14) into (A.13) for $\chi_{i}=1$, and using $z_{i}=0$, we can write the quantity that trader $i$ trades in equilibrium as

$$
x_{i}=\frac{\tau_{\zeta}\left(\tau_{\epsilon}+\tau_{\eta_{i}}+\tau_{\theta}\right)}{\alpha_{i}\left(\tau_{\epsilon}+\tau_{\zeta}+\tau_{\eta_{i}}+\tau_{\theta}\right)}\left(\sum_{j=1}^{N} a_{i j} s_{j}+b_{i} s\right),
$$


where

$$
\begin{aligned}
a_{i i} & \equiv \frac{\tau_{\eta_{i}}}{\tau_{\epsilon}+\tau_{\eta_{i}}+\tau_{\theta}}-A_{i}, \\
a_{i j} & \equiv-A_{j} \quad \text { for } j \neq i, \\
b_{i} & \equiv \frac{\tau_{\theta}}{\tau_{\epsilon}+\tau_{\eta_{i}}+\tau_{\theta}}-B .
\end{aligned}
$$

Using (1) and (2), we can write (A.36) as

$$
x_{i}=\frac{\tau_{\zeta}\left(\tau_{\epsilon}+\tau_{\eta_{i}}+\tau_{\theta}\right)}{\alpha_{i}\left(\tau_{\epsilon}+\tau_{\zeta}+\tau_{\eta_{i}}+\tau_{\theta}\right)}\left[\left(\sum_{j=1}^{N} a_{i j}+b_{i}\right) \epsilon+\sum_{j=1}^{N} a_{i j} \eta_{j}+b_{i} \theta\right] .
$$

Since $x_{i}$ is normal,

$$
\begin{aligned}
E\left(\left|x_{i}\right|\right) & =\sqrt{\frac{2 \operatorname{Var}\left(x_{i}\right)}{\pi}} \\
& =\frac{\tau_{\zeta}\left(\tau_{\epsilon}+\tau_{\eta_{i}}+\tau_{\theta}\right)}{\alpha_{i}\left(\tau_{\epsilon}+\tau_{\zeta}+\tau_{\eta_{i}}+\tau_{\theta}\right)} \sqrt{\frac{2}{\pi}\left[\left(\sum_{j=1}^{N} a_{i j}+b_{i}\right)^{2} \sigma_{\epsilon}^{2}+\sum_{j=1}^{N} a_{i j}^{2} \sigma_{\eta_{j}}^{2}+b_{i}^{2} \sigma_{\theta}^{2}\right]},
\end{aligned}
$$

where the second step follows from (A.40).

When $\alpha_{i}=\alpha$ and $\tau_{\eta_{i}}=\tau_{\eta}$ for all $i$, Proposition 3 implies that

$$
\begin{aligned}
A_{i} & =\frac{\tau_{\eta}}{N\left(\tau_{\epsilon}+\tau_{\eta}+\tau_{\theta}\right)} \quad \text { for all } i, \\
B & =\frac{\tau_{\theta}}{\tau_{\epsilon}+\tau_{\eta}+\tau_{\theta}} .
\end{aligned}
$$

Substituting into (A.37)-(A.39), we find

$$
\begin{aligned}
a_{i i} & =\frac{(N-1) \tau_{\eta}}{N\left(\tau_{\epsilon}+\tau_{\eta}+\tau_{\theta}\right)}, \\
a_{i j} & =-\frac{\tau_{\eta}}{N\left(\tau_{\epsilon}+\tau_{\eta}+\tau_{\theta}\right)} \text { for } j \neq i, \\
b_{i} & =0 \\
\sum_{j=1}^{N} a_{i j}+b_{i} & =0 .
\end{aligned}
$$

Substituting into (A.41), we find (23). The comparative statics follow by differentiating (23) with respect to $N$ and $\tau_{\eta}$. 
When $\alpha_{i}=\alpha$ for all $i$ and $\sigma_{\zeta}^{2}=0$, Proposition 3 implies that

$$
\begin{aligned}
A_{i} & =\frac{\tau_{\eta_{i}}}{N\left(\tau_{\epsilon}+\tau_{\eta}+\tau_{\theta}\right)} \quad \text { for all } i \\
B & =\frac{\tau_{\theta}}{\tau_{\epsilon}+\tau_{\eta}+\tau_{\theta}} .
\end{aligned}
$$

(These values can be derived as a limit when $\tau_{\zeta}$ goes to $\infty$.) Substituting into (A.37)-(A.39), we find

$$
\begin{aligned}
a_{i i} & =\frac{\left[(N-1)\left(\tau_{\epsilon}+\tau_{\theta}\right)+N \tau_{\eta}-\tau_{\eta_{i}}\right] \tau_{\eta_{i}}}{N\left(\tau_{\epsilon}+\tau_{\eta_{i}}+\tau_{\theta}\right)\left(\tau_{\epsilon}+\tau_{\eta}+\tau_{\theta}\right)}, \\
a_{i j} & =-\frac{\tau_{\eta_{j}}}{N\left(\tau_{\epsilon}+\tau_{\eta}+\tau_{\theta}\right)} \quad \text { for } j \neq i, \\
b_{i} & =\frac{\left(\tau_{\eta}-\tau_{\eta_{i}}\right) \tau_{\theta}}{\left(\tau_{\epsilon}+\tau_{\eta_{i}}+\tau_{\theta}\right)\left(\tau_{\epsilon}+\tau_{\eta}+\tau_{\theta}\right)}, \\
\sum_{j=1}^{N} a_{i j}+b_{i} & =\frac{\left(\tau_{\eta_{i}}-\tau_{\eta}\right) \tau_{\epsilon}}{\left(\tau_{\epsilon}+\tau_{\eta_{i}}+\tau_{\theta}\right)\left(\tau_{\epsilon}+\tau_{\eta}+\tau_{\theta}\right)} .
\end{aligned}
$$

Substituting into (A.41), and using again $\alpha_{i}=\alpha$ for all $i$ and $\sigma_{\zeta}^{2}=0$, we find

$E\left(\left|x_{i}\right|\right)=\frac{\sqrt{2\left[\left(\tau_{\eta_{i}}-\tau_{\eta}\right)^{2} \tau_{\epsilon}^{2} \sigma_{\epsilon}^{2}+\frac{\left[(N-1)\left(\tau_{\epsilon}+\tau_{\theta}\right)+N \tau_{\eta}-\tau_{\eta_{i}}\right]^{2} \tau_{\eta_{i}}^{2}}{N^{2}} \sigma_{\eta_{i}}^{2}+\sum_{j \neq i} \frac{\left(\tau_{\epsilon}+\tau_{\eta_{i}}+\tau_{\theta}\right)^{2} \tau_{\eta_{j}}^{2}}{N^{2}} \sigma_{\eta_{j}}^{2}+\left(\tau_{\eta}-\tau_{\eta_{i}}\right)^{2} \tau_{\theta}^{2} \sigma_{\theta}^{2}\right]}}{\alpha\left(\tau_{\epsilon}+\tau_{\eta}+\tau_{\theta}\right) \sqrt{\pi}}$.

Since the variance is the inverse of the precision, we can write (A.48) as

$$
\begin{aligned}
E\left(\left|x_{i}\right|\right)= & \frac{\sqrt{2\left[\left(\tau_{\eta_{i}}-\tau_{\eta}\right)^{2} \tau_{\epsilon}+\frac{\left[(N-1)\left(\tau_{\epsilon}+\tau_{\theta}\right)+N \tau_{\eta}-\tau_{\eta_{i}}\right]^{2} \tau_{\eta_{i}}}{N^{2}}+\sum_{j \neq i} \frac{\left(\tau_{\epsilon}+\tau_{\eta_{i}}+\tau_{\theta}\right)^{2} \tau_{\eta_{j}}}{N^{2}}+\left(\tau_{\eta}-\tau_{\eta_{i}}\right)^{2} \tau_{\theta}\right]}}{\alpha\left(\tau_{\epsilon}+\tau_{\eta}+\tau_{\theta}\right) \sqrt{\pi}} \\
= & \frac{\sqrt{2\left[\left(\tau_{\eta_{i}}-\tau_{\eta}\right)^{2}\left(\tau_{\epsilon}+\tau_{\theta}\right)+\frac{\left[(N-1)\left(\tau_{\epsilon}+\tau_{\theta}\right)+N \tau_{\eta}-\tau_{\eta_{i}}\right]^{2} \tau_{\eta_{i}}}{N^{2}}+\frac{\left(\tau_{\epsilon}+\tau_{\eta_{i}}+\tau_{\theta}\right)^{2}\left(N \tau_{\eta}-\tau_{\eta_{i}}\right)}{N^{2}}\right]}}{\alpha\left(\tau_{\epsilon}+\tau_{\eta}+\tau_{\theta}\right) \sqrt{\pi}}
\end{aligned}
$$

where the second step follows from the definition of $\tau_{\eta}$. Eq. (24) follows from (A.49) by separating quadratic, linear and constant terms in $\tau_{\epsilon}+\tau_{\eta}$. Trader $i$ generates more volume than trader $j$ if 
and only if the difference between the term inside the squared root in (24) and the corresponding term for $j$ is positive. The difference is

$$
\left[(N-2)\left(\tau_{\epsilon}+\tau_{\theta}\right)^{2}+(N-2)\left(\tau_{\epsilon}+\tau_{\theta}\right)\left(\tau_{\eta_{i}}+\tau_{\eta_{j}}\right)+\left(N \tau_{\eta}-\tau_{\eta_{i}}-\tau_{\eta_{j}}\right) \tau_{\eta}\right]\left(\tau_{\eta_{i}}-\tau_{\eta_{j}}\right) .
$$

Since

$$
N \tau_{\eta}-\tau_{\eta_{i}}-\tau_{\eta_{j}}=\sum_{k=1}^{N} \tau_{\eta_{k}}-\tau_{\eta_{i}}-\tau_{\eta_{j}}=\sum_{k \neq i, j} \tau_{\eta_{k}}>0,
$$

the difference is positive if and only if $\tau_{\eta_{i}}>\tau_{\eta_{j}}$

We next prove two lemmas, which we then use to prove Proposition 7.

Lemma A.2 Let $x$ be an $n \times 1$ normal vector with mean zero and covariance matrix $\Sigma, Z_{A} a$ scalar, $Z_{B}$ an $n \times 1$ vector, $Z_{C}$ an $n \times n$ symmetric matrix, $I$ the $n \times n$ identity matrix, $v^{\prime}$ the transpose of a vector $v$, and $|M|$ the determinant of a matrix $M$. Then,

$E_{x} \exp \left\{-\alpha\left[Z_{A}+Z_{B}^{\prime} x+\frac{1}{2} x^{\prime} Z_{C} x\right]\right\}=\exp \left\{-\alpha\left[Z_{A}-\frac{1}{2} \alpha Z_{B}^{\prime} \Sigma\left(I+\alpha Z_{C} \Sigma\right)^{-1} Z_{B}\right]\right\} \frac{1}{\sqrt{\left|I+\alpha Z_{C} \Sigma\right|}}$.

Proof. When $Z_{C}=0,($ A.50) gives the moment-generating function of the normal distribution. We can always assume $Z_{C}=0$ by also assuming that $x$ is a normal vector with mean 0 and covariance matrix $\Sigma\left(I+\alpha Z_{C} \Sigma\right)^{-1}$.

Lemma A.3 Suppose that the $n \times n$ matrix $Z_{C}$ is equal to $Z_{C 1} Z_{C 2}^{\prime}$ for $n \times 1$ vectors $\left(Z_{C 1}, Z_{C 2}\right)$, and the symmetric $n \times n$ matrix $\Sigma$ is positive definite. Then,

$$
\left|I+\alpha Z_{C} \Sigma\right|=1+\alpha Z_{C 1}^{\prime} \Sigma Z_{C 2} .
$$

Proof. Since $\Sigma$ is positive definite, it has a positive-definite square root. We denote that matrix by $\Sigma^{\frac{1}{2}}$ and its inverse by $\Sigma^{-\frac{1}{2}}$. We can write the determinant in (A.51) as

$$
\begin{aligned}
\left|I+\alpha Z_{C} \Sigma\right| & =\left|\left(\Sigma^{-\frac{1}{2}}+\alpha Z_{C} \Sigma^{\frac{1}{2}}\right) \Sigma^{\frac{1}{2}}\right| \\
& =\left|\Sigma^{\frac{1}{2}}\left(\Sigma^{-\frac{1}{2}}+\alpha Z_{C} \Sigma^{\frac{1}{2}}\right)\right| \\
& =\left|I+\alpha \Sigma^{\frac{1}{2}} Z_{C} \Sigma^{\frac{1}{2}}\right|
\end{aligned}
$$


where the second step follows because the determinant is commutative. We next compute

$$
\Delta \equiv\left|\Sigma^{\frac{1}{2}} Z_{C} \Sigma^{\frac{1}{2}}\left(I+\alpha \Sigma^{\frac{1}{2}} Z_{C} \Sigma^{\frac{1}{2}}\right)\right|
$$

in two different ways. First,

$$
\begin{aligned}
\Delta & =\left|\Sigma^{\frac{1}{2}} Z_{C} \Sigma^{\frac{1}{2}}\right|\left|I+\alpha \Sigma^{\frac{1}{2}} Z_{C} \Sigma^{\frac{1}{2}}\right| \\
& =\left|\Sigma^{\frac{1}{2}} Z_{C 1} Z_{C 2}^{\prime} \Sigma^{\frac{1}{2}}\right|\left|I+\alpha \Sigma^{\frac{1}{2}} Z_{C} \Sigma^{\frac{1}{2}}\right| \\
& =\left|Z_{C 2}^{\prime} \Sigma^{\frac{1}{2}} \Sigma^{\frac{1}{2}} Z_{C 1}\right|\left|I+\alpha \Sigma^{\frac{1}{2}} Z_{C} \Sigma^{\frac{1}{2}}\right| \\
& =Z_{C 2}^{\prime} \Sigma Z_{C 1}\left|I+\alpha \Sigma^{\frac{1}{2}} Z_{C} \Sigma^{\frac{1}{2}}\right|,
\end{aligned}
$$

where the first step follows because the determinant of a product is the product of the determinants, and the third step because the determinant is commutative. Second,

$$
\begin{aligned}
\Delta & =\left|\Sigma^{\frac{1}{2}} Z_{C 1} Z_{C 2}^{\prime} \Sigma^{\frac{1}{2}}\left(I+\alpha \Sigma^{\frac{1}{2}} Z_{C 1} Z_{C 2}^{\prime} \Sigma^{\frac{1}{2}}\right)\right| \\
& =\left|Z_{C 2}^{\prime} \Sigma^{\frac{1}{2}}\left(I+\alpha \Sigma^{\frac{1}{2}} Z_{C 1} Z_{C 2}^{\prime} \Sigma^{\frac{1}{2}}\right) \Sigma^{\frac{1}{2}} Z_{C 1}\right| \\
& =\left|Z_{C 2}^{\prime} \Sigma Z_{C 1}\left(1+\alpha Z_{C 2}^{\prime} \Sigma Z_{C 1}\right)\right| \\
& =Z_{C 2}^{\prime} \Sigma Z_{C 1}\left(1+\alpha Z_{C 2}^{\prime} \Sigma Z_{C 1}\right) .
\end{aligned}
$$

Comparing (A.53) to (A.54), and using (A.52), we find (A.51).

Proof of Proposition 7. We first derive a general expression for the expected utility of a fully cursed trader who receives no endowment shock. This derivation does not assume that all other traders are fully cursed, or that they have the same risk-aversion coefficient, or that $\sigma_{\zeta}^{2}=0$. We can write the expected utility of trader $i$ in the ex-ante sense as

$$
-\mathrm{E} \exp \left\{-\alpha_{i}\left[x_{i}(d-p)+z_{i} d\right]\right\}
$$


Using the law of iterative expectations, and denoting by $I \equiv\left(\left\{s_{j}\right\}_{j=1, . ., N}, s\right)$ the information set consisting of all the signals, we can write (A.55) as

$$
\begin{aligned}
& -\mathrm{E}\left[\mathrm{E}\left(\exp \left\{-\alpha_{i}\left[x_{i}(d-p)+z_{i} d\right]\right\} \mid I\right)\right] \\
& -\mathrm{E} \exp \left\{-\alpha_{i}\left[x_{i}(\mathrm{E}(d \mid I)-p)+z_{i} \mathrm{E}(d \mid I)-\frac{1}{2} \alpha_{i}\left(x_{i}+z_{i}\right)^{2} \operatorname{Var}(d \mid I)\right]\right\} \\
& -\mathrm{E} \exp \left\{-\alpha_{i}\left[\frac{\mathrm{E}\left(d \mid I_{i c}\right)-p}{\alpha_{i} \operatorname{Var}\left(d \mid I_{i c}\right)}\left(\mathrm{E}(d \mid I)-p-\frac{1}{2} \frac{\mathrm{E}\left(d \mid I_{i c}\right)-p}{\operatorname{Var}\left(d \mid I_{i c}\right)} \operatorname{Var}(d \mid I)\right)+z_{i} p\right]\right\} \\
& -\mathrm{E} \exp \left\{-\frac{\mathrm{E}\left(d \mid I_{i c}\right)-p}{\operatorname{Var}\left(d \mid I_{i c}\right)}\left(\mathrm{E}(d \mid I)-p-\frac{1}{2} \frac{\mathrm{E}\left(d \mid I_{i c}\right)-p}{\operatorname{Var}\left(d \mid I_{i c}\right)} \operatorname{Var}(d \mid I)\right)\right\}
\end{aligned}
$$

where the second step follows because of normality, the third from (8) and $\chi_{i}=1$, and the fourth because $z_{i}=0$. Using Lemma A.1 with $x=\epsilon, K=N+1$ and $\left\{y_{j}\right\}_{j=1, . ., N+1}=\left(\left\{\eta_{j}\right\}_{j=1, . ., N}, \eta\right)$, we find

$$
\begin{aligned}
\mathrm{E}(d \mid I) & =\bar{d}+\sum_{j=1}^{N} \frac{\tau_{\eta_{j}}}{\tau_{\epsilon}+\sum_{k=1}^{N} \tau_{\eta_{k}}+\tau_{\theta}} s_{j}+\frac{\tau_{\theta}}{\tau_{\epsilon}+\sum_{k=1}^{N} \tau_{\eta_{k}}+\tau_{\theta}} s \\
& =\bar{d}+\sum_{j=1}^{N} \frac{\tau_{\eta_{j}}}{\tau_{\epsilon}+N \tau_{\eta}+\tau_{\theta}} s_{j}+\frac{\tau_{\theta}}{\tau_{\epsilon}+N \tau_{\eta}+\tau_{\theta}} s, \\
\operatorname{Var}(d \mid I) & =\frac{\tau_{\epsilon}+\tau_{\zeta}+\sum_{j=1}^{N} \tau_{\eta_{j}}+\tau_{\theta}}{\left(\tau_{\epsilon}+\sum_{j=1}^{N} \tau_{\eta_{j}}+\tau_{\theta}\right) \tau_{\zeta}} \\
& =\frac{\tau_{\epsilon}+\tau_{\zeta}+N \tau_{\eta}+\tau_{\theta}}{\left(\tau_{\epsilon}+N \tau_{\eta}+\tau_{\theta}\right) \tau_{\zeta}} .
\end{aligned}
$$

Substituting conditional means and variances from (A.11), (A.12), (A.57) and (A.58), we can write the expected utility (A.56) as

$$
-\mathrm{E} \exp \left\{-\frac{1}{2} \frac{\tau_{\zeta}\left(\tau_{\epsilon}+\tau_{\eta_{i}}+\tau_{\theta}\right)}{\left(\tau_{\epsilon}+\tau_{\zeta}+\tau_{\eta_{i}}+\tau_{\theta}\right)}\left(\sum_{j=1}^{N} a_{i j} s_{j}+b_{i} s\right)\left(\sum_{j=1}^{N} \hat{a}_{i j} s_{j}+\hat{b}_{i} s\right)\right\},
$$

where $\left(\left\{a_{i j}\right\}_{j=1, . ., N}, b_{i}\right)$ are defined in (A.37)-(A.39), and

$$
\begin{aligned}
\hat{a}_{i j} & \equiv \frac{2 \tau_{\eta_{j}}}{\tau_{\epsilon}+N \tau_{\eta}+\tau_{\theta}}-2 A_{j}-\frac{\left(\tau_{\epsilon}+\tau_{\zeta}+N \tau_{\eta}+\tau_{\theta}\right)\left(\tau_{\epsilon}+\tau_{\eta_{i}}+\tau_{\theta}\right)}{\left(\tau_{\epsilon}+\tau_{\zeta}+\tau_{\eta_{i}}+\tau_{\theta}\right)\left(\tau_{\epsilon}+N \tau_{\eta}+\tau_{\theta}\right)} a_{i j} \quad \text { for } j=1, . ., N, \\
\hat{b}_{i} & \equiv \frac{2 \tau_{\theta}}{\tau_{\epsilon}+N \tau_{\eta}+\tau_{\theta}}-2 B-\frac{\left(\tau_{\epsilon}+\tau_{\zeta}+N \tau_{\eta}+\tau_{\theta}\right)\left(\tau_{\epsilon}+\tau_{\eta_{i}}+\tau_{\theta}\right)}{\left(\tau_{\epsilon}+\tau_{\zeta}+\tau_{\eta_{i}}+\tau_{\theta}\right)\left(\tau_{\epsilon}+N \tau_{\eta}+\tau_{\theta}\right)} b_{i} .
\end{aligned}
$$


Using (1) and (2), we can write (A.59) as

$$
-\mathrm{E} \exp \left\{-\frac{1}{2} \frac{\tau_{\zeta}\left(\tau_{\epsilon}+\tau_{\eta_{i}}+\tau_{\theta}\right)}{\left(\tau_{\epsilon}+\tau_{\zeta}+\tau_{\eta_{i}}+\tau_{\theta}\right)}\left[\left(\sum_{j=1}^{N} a_{i j}+b_{i}\right) \epsilon+\sum_{j=1}^{N} a_{i j} \eta_{j}+b_{i} \theta\right]\left[\left(\sum_{j=1}^{N} \hat{a}_{i j}+\hat{b}_{i}\right) \epsilon+\sum_{j=1}^{N} \hat{a}_{i j} \eta_{j}+\hat{b}_{i} \theta\right]\right\} .
$$

To compute the expectation in (A.62), we use Lemma A.2, and set

$$
\begin{aligned}
Z_{A} & \equiv 0, \\
Z_{B} & \equiv 0, \\
Z_{C} & \equiv \frac{\tau_{\zeta}\left(\tau_{\epsilon}+\tau_{\eta_{i}}+\tau_{\theta}\right)}{\alpha_{i}\left(\tau_{\epsilon}+\tau_{\zeta}+\tau_{\eta_{i}}+\tau_{\theta}\right)} v \hat{v}^{\prime}, \\
S i & \equiv \operatorname{Diag}\left(\sigma_{\epsilon}^{2},\left\{\sigma_{\eta_{j}}^{2}\right\}_{j=1, . ., N}, \sigma_{\theta}^{2}\right), \\
v & \equiv\left(\sum_{j=1}^{N} a_{i j}+b_{i},\left\{a_{i j}\right\}_{j=1, . ., N}, b_{i}\right)^{\prime}, \\
\hat{v} & \equiv\left(\sum_{j=1}^{N} \hat{a}_{i j}+\hat{b}_{i},\left\{\hat{a}_{i j}\right\}_{j=1, . ., N}, \hat{b}_{i}\right)^{\prime} .
\end{aligned}
$$

Lemma A.2 implies that (A.62) is equal to

$$
-\frac{1}{\sqrt{\left|I+\frac{\tau_{\zeta}\left(\tau_{\epsilon}+\tau_{\eta_{i}}+\tau_{\theta}\right)}{\left(\tau_{\epsilon}+\tau_{\zeta}+\tau_{\eta_{i}}+\tau_{\theta}\right)} v \hat{v}^{\prime} \Sigma\right|}}=-\frac{1}{\sqrt{1+\frac{\tau_{\zeta}\left(\tau_{\epsilon}+\tau_{\eta_{i}}+\tau_{\theta}\right)}{\left(\tau_{\epsilon}+\tau_{\zeta}+\tau_{\eta_{i}}+\tau_{\theta}\right)} v^{\prime} \Sigma \hat{v}}},
$$

where the second step follows from Lemma A.3.

We next use (A.63) to compute the expected utility of a fully cursed trader under the assumptions in the proposition. When $\alpha_{i}=\alpha$ for all $i$ and $\sigma_{\zeta}^{2}=0,\left(\left\{a_{i j}\right\}_{j=1, . ., N}, b_{i}\right)$ are given by (A.44)-(A.46). Moreover, substituting $\left(\left\{A_{j}\right\}_{j=1, . ., N}, A,\left\{a_{i j}\right\}_{j=1, . ., N}, b_{i}\right)$ from (A.42)-(A.46) into 
(A.60) and (A.61), and using $\alpha_{i}=\alpha$ for all $i$ and $\sigma_{\zeta}^{2}=0$, we find

$$
\begin{aligned}
\hat{a}_{i i} & =\frac{\left[(N-1)\left(\tau_{\epsilon}+\tau_{\theta}\right)-N \tau_{\eta}+\tau_{\eta_{i}}\right] \tau_{\eta_{i}}}{N\left(\tau_{\epsilon}+\tau_{\eta}+\tau_{\theta}\right)\left(\tau_{\epsilon}+N \tau_{\eta}+\tau_{\theta}\right)}, \\
\hat{a}_{i j} & =\frac{\left[(2 N-1)\left(\tau_{\epsilon}+\tau_{\theta}\right)+\tau_{\eta_{i}}\right] \tau_{\eta_{j}}}{N\left(\tau_{\epsilon}+\tau_{\eta}+\tau_{\theta}\right)\left(\tau_{\epsilon}+N \tau_{\eta}+\tau_{\theta}\right)} \text { for } j \neq i, \\
\hat{b}_{i} & =\frac{\left[-(2 N-1) \tau_{\eta}+\tau_{\eta_{i}}\right] \tau_{\theta}}{\left(\tau_{\epsilon}+\tau_{\eta}+\tau_{\theta}\right)\left(\tau_{\epsilon}+N \tau_{\eta}+\tau_{\theta}\right)}, \\
\sum_{j=1}^{N} \hat{a}_{i j}+\hat{b}_{i} & =\frac{\left[(2 N-1) \tau_{\eta}-\tau_{\eta_{i}}\right] \tau_{\epsilon}}{\left(\tau_{\epsilon}+\tau_{\eta}+\tau_{\theta}\right)\left(\tau_{\epsilon}+N \tau_{\eta}+\tau_{\theta}\right)} .
\end{aligned}
$$

Substituting into (A.63), and using again $\sigma_{\zeta}^{2}=0$, we can write the term inside the squared root as

$$
\begin{aligned}
& 1+\frac{\left(\tau_{\eta_{i}}-\tau_{\eta}\right)\left[(2 N-1) \tau_{\eta}-\tau_{\eta_{i}}\right] \tau_{\epsilon}^{2}}{\left(\tau_{\epsilon}+\tau_{\eta}+\tau_{\theta}\right)^{2}\left(\tau_{\epsilon}+N \tau_{\eta}+\tau_{\theta}\right)} \sigma_{\epsilon}^{2} \\
& +\frac{\left[(N-1)\left(\tau_{\epsilon}+\tau_{\theta}\right)+N \tau_{\eta}-\tau_{\eta_{i}}\right]\left[(N-1)\left(\tau_{\epsilon}+\tau_{\theta}\right)-N \tau_{\eta}+\tau_{\eta_{i}}\right] \tau_{\eta_{i}}^{2}}{N^{2}\left(\tau_{\epsilon}+\tau_{\eta}+\tau_{\theta}\right)^{2}\left(\tau_{\epsilon}+N \tau_{\eta}+\tau_{\theta}\right)} \sigma_{\eta_{i}}^{2} \\
& -\sum_{j \neq i} \frac{\left(\tau_{\epsilon}+\tau_{\eta_{i}}+\tau_{\theta}\right)\left[(2 N-1)\left(\tau_{\epsilon}+\tau_{\theta}\right)+\tau_{\eta_{i}}\right] \tau_{\eta_{j}}^{2}}{N^{2}\left(\tau_{\epsilon}+\tau_{\eta}+\tau_{\theta}\right)^{2}\left(\tau_{\epsilon}+N \tau_{\eta}+\tau_{\theta}\right)} \sigma_{\eta_{j}}^{2}+\frac{\left(\tau_{\eta}-\tau_{\eta_{i}}\right)\left[-(2 N-1) \tau_{\eta}+\tau_{\eta_{i}}\right] \tau_{\theta}^{2}}{\left(\tau_{\epsilon}+\tau_{\eta}+\tau_{\theta}\right)^{2}\left(\tau_{\epsilon}+N \tau_{\eta}+\tau_{\theta}\right)} \sigma_{\theta}^{2} .
\end{aligned}
$$

Eq. (25) can be derived from (A.68) by following the same steps as when deriving (24) from (A.48). Trader $i$ has higher expected utility than trader $j$ if and only if the difference between the numerator inside the squared root in (25) and the corresponding term for $j$ is positive. The difference is $\mathcal{Z}\left(\tau_{\eta_{i}}-\tau_{\eta_{j}}\right)$, where

$$
\mathcal{Z} \equiv N\left(\tau_{\epsilon}+\tau_{\theta}\right)^{2}+\left(\tau_{\epsilon}+\tau_{\theta}\right)\left[2 N(N+1) \tau_{\eta}-(N-2)\left(\tau_{\eta_{i}}+\tau_{\eta_{j}}\right)\right]-\left(N \tau_{\eta}-\tau_{\eta_{i}}-\tau_{\eta_{j}}\right) \tau_{\eta}
$$

Since $\mathcal{Z}$ is linear in $\tau_{\eta_{i}}+\tau_{\eta_{j}} \in\left(0, N \tau_{\eta}\right)$, it is positive if this is the case at the boundaries $\tau_{\eta_{i}}+\tau_{\eta_{j}}=0$ and $\tau_{\eta_{i}}+\tau_{\eta_{j}}=N \tau_{\eta}$. For $\tau_{\eta_{i}}+\tau_{\eta_{j}}=N \tau_{\eta}$,

$$
\mathcal{Z}=N\left(\tau_{\epsilon}+\tau_{\theta}\right)^{2}+N(N+4)\left(\tau_{\epsilon}+\tau_{\theta}\right) \tau_{\eta}>0 .
$$

For $\tau_{\eta_{i}}+\tau_{\eta_{j}}=0$

$$
\mathcal{Z}=N\left(\tau_{\epsilon}+\tau_{\theta}\right)^{2}+2 N(N+1)\left(\tau_{\epsilon}+\tau_{\theta}\right) \tau_{\eta}-N \tau_{\eta}^{2},
$$


and is positive if (26) holds. Therefore, if (26) holds, trader $i$ has higher expected utility than trader $j$ if and only if $\tau_{\eta_{i}}>\tau_{\eta_{j}}$. If instead (26) does not hold, $\mathcal{Z}<0$ for $\tau_{\eta_{i}}+\tau_{\eta_{j}}=0$, and there exist $\tau_{\eta_{i}}>\tau_{\eta_{j}}$ such that trader $i$ has lower expected utility than trader $j$.

Proof of Proposition 8. Setting $\left(\tau_{\eta_{i}}, A_{i}\right)=\left(\tau_{\eta}, A\right)$ in (A.16), we find $\tau_{\xi_{i}}=(N-1) \tau_{\eta}$. Setting $\left(\chi_{i}, \alpha_{i}, \tau_{\eta_{i}}, \tau_{\xi_{i}}, A_{i}\right)=\left(\chi, \alpha, \tau_{\eta},(N-1) \tau_{\eta}, A\right)$ for all $i$ in (11) and (12), we find (28) and (29), respectively. The comparative statics of $(A, B)$ follow from (28) and (29).

Substituting $p$ from (27) into (A.13), and using symmetry and $z_{i}=0$, we can write the quantity that trader $i$ trades in equilibrium as

$$
x_{i}=\frac{\tau_{\zeta}\left(\tau_{\epsilon}+\tau_{\eta}+\tau_{\theta}\right)\left(\tau_{\epsilon}+N \tau_{\eta}+\tau_{\theta}\right)}{\alpha\left(\tau_{\zeta}\left\{\tau_{\epsilon}+[1+\chi(N-1)] \tau_{\eta}+\tau_{\theta}\right\}+\left(\tau_{\epsilon}+\tau_{\eta}+\tau_{\theta}\right)\left(\tau_{\epsilon}+N \tau_{\eta}+\tau_{\theta}\right)\right)}\left(\sum_{j=1}^{N} a_{i j} s_{j}+b_{i} s\right),
$$

where

$$
\begin{aligned}
a_{i i} & \equiv \frac{\tau_{\eta}\left\{\tau_{\epsilon}+[1+\chi(N-1)] \tau_{\eta}+\tau_{\theta}\right\}}{\left(\tau_{\epsilon}+\tau_{\eta}+\tau_{\theta}\right)\left(\tau_{\epsilon}+N \tau_{\eta}+\tau_{\theta}\right)}-A, \\
a_{i j} & \equiv \frac{(1-\chi) \tau_{\eta}}{\tau_{\epsilon}+N \tau_{\eta}+\tau_{\theta}}-A, \quad \text { for } j \neq i, \\
b_{i} & \equiv \frac{\tau_{\theta}\left\{\tau_{\epsilon}+[1+\chi(N-1)] \tau_{\eta}+\tau_{\theta}\right\}}{\left(\tau_{\epsilon}+\tau_{\eta}+\tau_{\theta}\right)\left(\tau_{\epsilon}+N \tau_{\eta}+\tau_{\theta}\right)}-B .
\end{aligned}
$$

Proceeding as in the proof of Proposition 6, we find

$$
\begin{aligned}
E\left(\left|x_{i}\right|\right) & =\frac{\tau_{\zeta}\left(\tau_{\epsilon}+\tau_{\eta}+\tau_{\theta}\right)\left(\tau_{\epsilon}+N \tau_{\eta}+\tau_{\theta}\right)}{\alpha\left(\tau_{\zeta}\left\{\tau_{\epsilon}+[1+\chi(N-1)] \tau_{\eta}+\tau_{\theta}\right\}+\left(\tau_{\epsilon}+\tau_{\eta}+\tau_{\theta}\right)\left(\tau_{\epsilon}+N \tau_{\eta}+\tau_{\theta}\right)\right)} \\
& \times \sqrt{\frac{2}{\pi}\left[\left(\sum_{j=1}^{N} a_{i j}+b_{i}\right)^{2} \sigma_{\epsilon}^{2}+\sum_{j=1}^{N} a_{i j}^{2} \sigma_{\eta_{j}}^{2}+b_{i}^{2} \sigma_{\theta}^{2}\right]} .
\end{aligned}
$$


Substituting $(A, B)$ from (28) and (29) into (A.71)-(A.73), we find

$$
\begin{aligned}
a_{i i} & =\frac{\chi(N-1) \tau_{\eta}}{N\left(\tau_{\epsilon}+\tau_{\eta}+\tau_{\theta}\right)}, \\
a_{i j} & =-\frac{\chi \tau_{\eta}}{N\left(\tau_{\epsilon}+\tau_{\eta}+\tau_{\theta}\right)}, \quad \text { for } j \neq i, \\
b_{i} & =0, \\
\sum_{j=1}^{N} a_{i j}+b_{i} & =0 .
\end{aligned}
$$

Substituting into (A.74), we find (30). The comparative statics with respect to $\chi$ follow from (30). The comparative statics with respect to $N$ also follow from (30) by writing the term multiplying the squared root as

$$
\frac{\chi \tau_{\zeta}}{\alpha\left(\tau_{\zeta} \frac{\tau_{\epsilon}+[1+\chi(N-1)] \tau_{\eta}+\tau_{\theta}}{\tau_{\epsilon}+N \tau_{\eta}+\tau_{\theta}}+\tau_{\epsilon}+\tau_{\eta}+\tau_{\theta}\right)}
$$

and noting that

$$
\frac{\tau_{\epsilon}+[1+\chi(N-1)] \tau_{\eta}+\tau_{\theta}}{\tau_{\epsilon}+N \tau_{\eta}+\tau_{\theta}}
$$

decreases in $N$.

Proof of Proposition 9. Setting $\left(\tau_{\eta_{i}}, A_{i}\right)=\left(\tau_{\eta r}, A_{r}\right)$ for $i \in R$ and $\left(\tau_{\eta_{i}}, A_{i}\right)=\left(\tau_{\eta c}, A_{c}\right)$ for $i \in C$ in (A.16), we find

$$
\tau_{\xi_{i}}=\frac{\left[\left(N_{r}-1\right) A_{r}+N_{c} A_{c}\right]^{2} \tau_{\eta r} \tau_{\eta c}}{\left(N_{r}-1\right) A_{r}^{2} \tau_{\eta c}+N_{c} A_{c}^{2} \tau_{\eta r}} \equiv \tau_{\xi}
$$

for $i \in R$. We next set $\left(\chi_{i}, \alpha_{i}, \tau_{\eta_{i}}, \tau_{\xi_{i}}, A_{i}\right)=\left(0, \alpha_{r}, \tau_{\eta r}, \tau_{\xi}, A_{r}\right)$ for $i \in R$ and $\left(\chi_{i}, \alpha_{i}, \tau_{\eta_{i}}, A_{i}\right)=$ $\left(1, \alpha_{c}, \tau_{\eta c}, A_{c}\right)$ for $i \in C$ in (11) and (12). Equation (11) written for a rational trader becomes $\frac{\tau_{\eta r}-\tau_{\xi} \frac{A_{r}}{\alpha_{r}\left(N_{\epsilon}-1\right) A_{r}+N_{c} A_{c}}}{\left.\alpha_{\zeta}+\tau_{\eta r}+\tau_{\theta}+\tau_{\xi}\right)}=A_{r}\left[\frac{N_{r}\left(\tau_{\epsilon}+\tau_{\eta r}+\tau_{\theta}+\tau_{\xi}-\tau_{\xi} \frac{1}{\left(N_{r}-1\right) A_{r}+N_{c} A_{c}}\right)}{\alpha_{r}\left(\tau_{\epsilon}+\tau_{\zeta}+\tau_{\eta r}+\tau_{\theta}+\tau_{\xi}\right)}+\frac{N_{c}\left(\tau_{\epsilon}+\tau_{\eta c}+\tau_{\theta}\right)}{\alpha_{c}\left(\tau_{\epsilon}+\tau_{\zeta}+\tau_{\eta c}+\tau_{\theta}\right)}\right]$,

and written for a fully cursed trader becomes

$$
\frac{\tau_{\eta c}}{\alpha_{c}\left(\tau_{\epsilon}+\tau_{\zeta}+\tau_{\eta c}+\tau_{\theta}\right)}=A_{c}\left[\frac{N_{r}\left(\tau_{\epsilon}+\tau_{\eta r}+\tau_{\theta}+\tau_{\xi}-\tau_{\xi} \frac{1}{\left(N_{r}-1\right) A_{r}+N_{c} A_{c}}\right)}{\alpha_{r}\left(\tau_{\epsilon}+\tau_{\zeta}+\tau_{\eta r}+\tau_{\theta}+\tau_{\xi}\right)}+\frac{N_{c}\left(\tau_{\epsilon}+\tau_{\eta c}+\tau_{\theta}\right)}{\alpha_{c}\left(\tau_{\epsilon}+\tau_{\zeta}+\tau_{\eta c}+\tau_{\theta}\right)}\right] .
$$


Equation (12) becomes

$$
\begin{aligned}
& \frac{N_{r} \tau_{\theta}}{\alpha_{r}\left(\tau_{\epsilon}+\tau_{\zeta}+\tau_{\eta r}+\tau_{\theta}+\tau_{\xi}\right)}+\frac{N_{c} \tau_{\theta}}{\alpha_{c}\left(\tau_{\epsilon}+\tau_{\zeta}+\tau_{\eta c}+\tau_{\theta}\right)} \\
& =B\left[\frac{N_{r}\left(\tau_{\epsilon}+\tau_{\eta r}+\tau_{\theta}+\tau_{\xi}\right)}{\alpha_{r}\left(\tau_{\epsilon}+\tau_{\zeta}+\tau_{\eta r}+\tau_{\theta}+\tau_{\xi}\right)}+\frac{N_{c}\left(\tau_{\epsilon}+\tau_{\eta c}+\tau_{\theta}\right)}{\alpha_{c}\left(\tau_{\epsilon}+\tau_{\zeta}+\tau_{\eta c}+\tau_{\theta}\right)}\right] .
\end{aligned}
$$

Equations (A.76) and (A.77) form a system of two equations in the two unknowns $\left(A_{r}, A_{c}\right)$. We can reduce this system to one equation by setting $x \equiv \frac{A_{r}}{A_{c}}$. Equation (A.75) then becomes (35), and (A.77) and (A.78) yield (33) and (34), respectively. Moreover, dividing (32) by (33), we find

$$
\begin{gathered}
\frac{\tau_{\eta r}-\tau_{\xi} \frac{x}{\left(N_{r}-1\right) x+N_{c}}}{\alpha_{r}\left(\tau_{\epsilon}+\tau_{\zeta}+\tau_{\eta r}+\tau_{\theta}+\tau_{\xi}\right)} \frac{\alpha_{c}\left(\tau_{\epsilon}+\tau_{\zeta}+\tau_{\eta c}+\tau_{\theta}\right)}{\tau_{\eta c}}=x \\
\Rightarrow x=\frac{\alpha_{c}\left\{\tau_{\eta r}-\frac{x\left[\left(N_{r}-1\right) x+N_{c}\right] \tau_{\eta r} \tau_{\eta c}}{\left(N_{r}-1\right) x^{2} \tau_{\eta c}+N_{c} \tau_{\eta r}}\right\}\left(\tau_{\epsilon}+\tau_{\zeta}+\tau_{\eta c}+\tau_{\theta}\right)}{\alpha_{r} \tau_{\eta c}\left\{\tau_{\epsilon}+\tau_{\zeta}+\tau_{\eta r}+\tau_{\theta}+\frac{\left[\left(N_{r}-1\right) x+N_{c}\right]^{2} \tau_{\eta r} \tau_{\eta c}}{\left(N_{r}-1\right) x^{2} \tau_{\eta c}+N_{c} \tau_{\eta r}}\right\}},
\end{gathered}
$$

where the second step follows by (35). Equation (A.79) yields (36).

For $x \leq 0$, the left-hand side of (36) is non-positive and the right-hand side is positive. For $x \geq \frac{\tau_{\eta r}}{\tau_{\eta c}}$ the left-hand side of (36) is positive and the right-hand side is non-positive. Therefore, a solution of (36) must belong to $\left(0, \frac{\tau_{\eta r}}{\tau_{\eta c}}\right)$. For $x \in\left(0, \frac{\tau_{\eta r}}{\tau_{\eta c}}\right)$, the left-hand side of (36) is increasing in $x$ and the right-hand side is decreasing in $x$ (because the numerator is decreasing, the denominator is increasing, and both are positive). Since the left-hand side is zero at $x=0$, and the right-hand side is zero at $x=\frac{\tau_{\eta r}}{\tau_{\eta c}}$, a solution of (36) exists and is unique.

Proof of Proposition 10. Because of symmetry, the coefficient $\gamma_{i}$ is equal to a common value $\gamma_{r}$ for all rational traders and to a common value $\gamma_{c}$ for all fully cursed traders. Market clearing (5) implies that $N_{r} \gamma_{r}+N_{c} \gamma_{c}=0$. Hence, to prove the proposition it suffices to show that $\gamma_{c}<0$.

The coefficient $\gamma_{c}$ is proportional to $\operatorname{Cov}\left(x_{i}, p-\bar{d}\right)$. Substituting $p$ from (31) into (A.13), and setting $\left(\chi_{i}, \tau_{\eta_{i}}, z_{i}\right)=\left(1, \tau_{\eta c}, 0\right)$, we find that $\operatorname{Cov}\left(x_{i}, p-\bar{d}\right)$ has the same sign as

$$
\operatorname{Cov}\left(\bar{d}+\frac{\tau_{\eta c} s_{i}+\tau_{\theta} s}{\tau_{\epsilon}+\tau_{\eta c}+\tau_{\theta}}-p \cdot p-\bar{d}\right)
$$


Using (1), (2) and (31), we find that $\operatorname{Cov}\left(x_{i}, p-\bar{d}\right)$ has the same sign as

$$
\begin{aligned}
& \left(\frac{\tau_{\eta c}+\tau_{\theta}}{\tau_{\epsilon}+\tau_{\eta c}+\tau_{\theta}}-N_{r} A_{r}-N_{c} A_{c}-B\right)\left(N_{r} A_{r}+N_{c} A_{c}+B\right) \sigma_{\epsilon}^{2} \\
& +\left(\frac{\tau_{\eta c}}{\tau_{\epsilon}+\tau_{\eta c}+\tau_{\theta}}-N_{c} A_{c}\right) A_{c} \sigma_{\eta c}^{2}-N_{r} A_{r}^{2} \sigma_{\eta r}^{2}+\left(\frac{\tau_{\theta}}{\tau_{\epsilon}+\tau_{\eta c}+\tau_{\theta}}-B\right) B \sigma_{\theta}^{2},
\end{aligned}
$$

where $\left(\sigma_{\eta r}^{2}, \sigma_{\eta c}^{2}\right) \equiv\left(\frac{1}{\tau_{\eta r}}, \frac{1}{\tau_{\eta c}}\right)$. To determine the sign of $(\mathrm{A} .81)$, we compute some of the terms in that equation.

Multiplying (A.76) by $N_{r}$, (A.77) by $N_{c}$, and adding the sum of the resulting equations to (A.78), we find

$$
\begin{aligned}
\left(N_{r} A_{r}+N_{c} A_{c}+B\right) \mathcal{D} & =\frac{N_{r}\left(\tau_{\eta r}+\tau_{\theta}\right)+N_{r} \tau_{\xi} \frac{\left(N_{r}-1\right) A_{r}+N_{c} A_{c}}{\left(N_{r}-1\right) A_{r}+N_{c} A_{c}}}{\alpha_{r}\left(\tau_{\epsilon}+\tau_{\zeta}+\tau_{\eta r}+\tau_{\theta}+\tau_{\xi}\right)}+\frac{N_{c}\left(\tau_{\eta c}+\tau_{\theta}\right)}{\alpha_{c}\left(\tau_{\epsilon}+\tau_{\zeta}+\tau_{\eta c}+\tau_{\theta}\right)} \\
& =\frac{N_{r}\left(\tau_{\eta r}+\tau_{\theta}+\tau_{\xi}\right)}{\alpha_{r}\left(\tau_{\epsilon}+\tau_{\zeta}+\tau_{\eta r}+\tau_{\theta}+\tau_{\xi}\right)}+\frac{N_{c}\left(\tau_{\eta c}+\tau_{\theta}\right)}{\alpha_{c}\left(\tau_{\epsilon}+\tau_{\zeta}+\tau_{\eta c}+\tau_{\theta}\right)},
\end{aligned}
$$

where

$$
\mathcal{D} \equiv \frac{N_{r}\left(\tau_{\epsilon}+\tau_{\eta r}+\tau_{\theta}+\tau_{\xi}\right)}{\alpha_{r}\left(\tau_{\epsilon}+\tau_{\zeta}+\tau_{\eta r}+\tau_{\theta}+\tau_{\xi}\right)}+\frac{N_{c}\left(\tau_{\epsilon}+\tau_{\eta c}+\tau_{\theta}\right)}{\alpha_{c}\left(\tau_{\epsilon}+\tau_{\zeta}+\tau_{\eta c}+\tau_{\theta}\right)}
$$

Therefore,

$$
\begin{aligned}
\frac{\tau_{\eta c}+\tau_{\theta}}{\tau_{\epsilon}+\tau_{\eta c}+\tau_{\theta}}-N_{r} A_{r}-N_{c} A_{c}-B & =\frac{\tau_{\eta c}+\tau_{\theta}}{\tau_{\epsilon}+\tau_{\eta c}+\tau_{\theta}}-\frac{\frac{N_{r}\left(\tau_{\eta r}+\tau_{\theta}+\tau_{\xi}\right)}{\alpha_{r}\left(\tau_{\epsilon}+\tau_{\zeta}+\tau_{\eta r}+\tau_{\theta}+\tau_{\xi}\right)}+\frac{N_{c}\left(\tau_{\eta c}+\tau_{\theta}\right)}{\alpha_{c}\left(\tau_{\epsilon}+\tau_{\zeta}+\tau_{\eta c}+\tau_{\theta}\right)}}{\mathcal{D}} \\
& =\frac{\frac{N_{r}\left(\tau_{\eta c}+\tau_{\theta}\right)\left(\tau_{\epsilon}+\tau_{\eta r}+\tau_{\theta}+\tau_{\xi}\right)}{\tau_{\epsilon}+\tau_{\eta c}+\tau_{\theta}}-N_{r}\left(\tau_{\eta r}+\tau_{\theta}+\tau_{\xi}\right)}{\alpha_{r}\left(\tau_{\epsilon}+\tau_{\zeta}+\tau_{\eta r}+\tau_{\theta}+\tau_{\xi}\right) \mathcal{D}} \\
& =\frac{N_{r} \tau_{\epsilon}\left(\tau_{\eta c}-\tau_{\eta r}-\tau_{\xi}\right)}{\alpha_{r}\left(\tau_{\epsilon}+\tau_{\zeta}+\tau_{\eta r}+\tau_{\theta}+\tau_{\xi}\right)\left(\tau_{\epsilon}+\tau_{\eta c}+\tau_{\theta}\right) \mathcal{D}}
\end{aligned}
$$

Multiplying (A.76) by $\frac{N_{r} A_{r}}{\tau_{\eta r}}$, (A.77) by $\frac{N_{c} A_{c}}{\tau_{\eta c}}$, and adding the resulting equations, we find

$$
\begin{aligned}
\left(\frac{N_{r} A_{r}^{2}}{\tau_{\eta r}}+\frac{N_{c} A_{c}^{2}}{\tau_{\eta c}}\right) \mathcal{D} & =\frac{N_{r} A_{r}+N_{r} \tau_{\xi} \frac{\left(N_{r}-1\right) \frac{A_{r}^{2}}{\tau_{\eta r}}+\frac{N_{c} A_{c}^{2}}{\tau_{\eta c}}}{\alpha_{r}\left(\tau_{\epsilon}+\tau_{\zeta}+\tau_{\eta r}+\tau_{\theta}+\tau_{\xi}\right)}}{\left.\tau_{\xi}\right)}+\frac{N_{c} A_{c}}{\alpha_{c}\left(\tau_{\epsilon}+\tau_{\zeta}+\tau_{\eta c}+\tau_{\theta}\right)} \\
& =\frac{N_{r} A_{r}+N_{r}\left[\left(N_{r}-1\right) A_{r}+N_{c} A_{c}\right]}{\alpha_{r}\left(\tau_{\epsilon}+\tau_{\zeta}+\tau_{\eta r}+\tau_{\theta}+\tau_{\xi}\right)}+\frac{N_{c} A_{c}}{\alpha_{c}\left(\tau_{\epsilon}+\tau_{\zeta}+\tau_{\eta c}+\tau_{\theta}\right)} \\
& =\frac{N_{r}\left(N_{r} A_{r}+N_{c} A_{c}\right)}{\alpha_{r}\left(\tau_{\epsilon}+\tau_{\zeta}+\tau_{\eta r}+\tau_{\theta}+\tau_{\xi}\right)}+\frac{N_{c} A_{c}}{\alpha_{c}\left(\tau_{\epsilon}+\tau_{\zeta}+\tau_{\eta c}+\tau_{\theta}\right)},
\end{aligned}
$$


where the second step follows from (A.75). Therefore,

$$
\begin{aligned}
\left(\frac{\tau_{\eta c}}{\tau_{\epsilon}+\tau_{\eta c}+\tau_{\theta}}-N_{c} A_{c}\right) A_{c} \sigma_{\eta c}^{2}-N_{r} A_{r}^{2} \sigma_{\eta r}^{2} & =\frac{A_{c}}{\tau_{\epsilon}+\tau_{\eta c}+\tau_{\theta}}-\frac{\frac{N_{r}\left(N_{r} A_{r}+N_{c} A_{c}\right)}{\alpha_{r}\left(\tau_{\epsilon}+\tau_{\zeta}+\tau_{\eta r}+\tau_{\theta}+\tau_{\xi}\right)}+\frac{N_{c} A_{c}}{\alpha_{c}\left(\tau_{\epsilon}+\tau_{\zeta}+\tau_{\eta c}+\tau_{\theta}\right)}}{\mathcal{D}} \\
& =\frac{\frac{N_{r}\left(\tau_{\epsilon}+\tau_{\eta r}+\tau_{\theta}+\tau_{\xi}\right) A_{c}}{\tau_{\epsilon}+\tau_{\eta c}+\tau_{\theta}}-N_{r}\left(N_{r} A_{r}+N_{c} A_{c}\right)}{\alpha_{r}\left(\tau_{\epsilon}+\tau_{\zeta}+\tau_{\eta r}+\tau_{\theta}+\tau_{\xi}\right) \mathcal{D}} .
\end{aligned}
$$

Using (34), we find

$$
\begin{aligned}
\frac{\tau_{\theta}}{\tau_{\epsilon}+\tau_{\eta c}+\tau_{\theta}}-B & =\frac{\tau_{\theta}}{\tau_{\epsilon}+\tau_{\eta c}+\tau_{\theta}}-\frac{\frac{N_{r} \tau_{\theta}}{\alpha_{r}\left(\tau_{\epsilon}+\tau_{\zeta}+\tau_{\eta r}+\tau_{\theta}+\tau_{\xi}\right)}+\frac{N_{c} \tau_{\theta}}{\alpha_{c}\left(\tau_{\epsilon}+\tau_{\zeta}+\tau_{\eta c}+\tau_{\theta}\right)}}{\mathcal{D}} \\
& =\frac{\frac{N_{r} \tau_{\theta}\left(\tau_{\epsilon}+\tau_{\eta r}+\tau_{\theta}+\tau_{\xi}\right)}{\tau_{\epsilon}+\tau_{\eta c}+\tau_{\theta}}-N_{r} \tau_{\theta}}{\alpha_{r}\left(\tau_{\epsilon}+\tau_{\zeta}+\tau_{\eta r}+\tau_{\theta}+\tau_{\xi}\right) \mathcal{D}} \\
& =\frac{N_{r} \tau_{\theta}\left(\tau_{\eta r}+\tau_{\xi}-\tau_{\eta c}\right)}{\alpha_{r}\left(\tau_{\epsilon}+\tau_{\zeta}+\tau_{\eta r}+\tau_{\theta}+\tau_{\xi}\right)\left(\tau_{\epsilon}+\tau_{\eta c}+\tau_{\theta}\right) \mathcal{D}} .
\end{aligned}
$$

Equations (A.82), (A.83) and (A.84) imply that (A.81) is equal to

$$
\begin{aligned}
& \frac{N_{r}\left(\tau_{\eta c}-\tau_{\eta r}-\tau_{\xi}\right)\left(N_{r} A_{r}+N_{c} A_{c}+B\right)}{\alpha_{r}\left(\tau_{\epsilon}+\tau_{\zeta}+\tau_{\eta r}+\tau_{\theta}+\tau_{\xi}\right)\left(\tau_{\epsilon}+\tau_{\eta c}+\tau_{\theta}\right) \mathcal{D}} \\
& +\frac{\frac{N_{r}\left(\tau_{\epsilon}+\tau_{\eta r}+\tau_{\theta}+\tau_{\xi}\right) A_{c}}{\tau_{\epsilon}+\tau_{\eta c}+\tau_{\theta}}-N_{r}\left(N_{r} A_{r}+N_{c} A_{c}\right)}{\alpha_{r}\left(\tau_{\epsilon}+\tau_{\zeta}+\tau_{\eta r}+\tau_{\theta}+\tau_{\xi}\right) \mathcal{D}}+\frac{N_{r}\left(\tau_{\eta r}+\tau_{\xi}-\tau_{\eta c}\right) B}{\alpha_{r}\left(\tau_{\epsilon}+\tau_{\zeta}+\tau_{\eta r}+\tau_{\theta}+\tau_{\xi}\right)\left(\tau_{\epsilon}+\tau_{\eta c}+\tau_{\theta}\right) \mathcal{D}} \\
& =\frac{N_{r}\left(\tau_{\epsilon}+\tau_{\eta r}+\tau_{\theta}+\tau_{\xi}\right)\left(A_{c}-N_{r} A_{r}-N_{c} A_{c}\right)}{\alpha_{r}\left(\tau_{\epsilon}+\tau_{\zeta}+\tau_{\eta r}+\tau_{\theta}+\tau_{\xi}\right)\left(\tau_{\epsilon}+\tau_{\eta c}+\tau_{\theta}\right) \mathcal{D}} .
\end{aligned}
$$

This is negative because $N_{c} \geq 1, A_{r}>0$ and $A_{c}>0$.

Proof of Proposition 11. Expected aggregate trading volume when all $N$ traders are fully cursed is $N$ times the expected volume that each trader generates, given by (23). To compute expected aggregate trading volume when one trader is rational and $N-1$ traders are fully cursed, we start by computing the expected trading volume that one fully cursed trader generates. Substituting $p$ from (31) into (A.13), setting $\left(\chi_{i}, \alpha_{i}, \tau_{\eta_{i}}, z_{i}\right)=\left(1, \alpha, \tau_{\eta}, 0\right)$, and denoting the rational trader by $i_{r}$, we can write the quantity that trader $i \neq i_{r}$ trades in equilibrium as

$$
x_{i}=\frac{\tau_{\zeta}\left(\tau_{\epsilon}+\tau_{\eta}+\tau_{\theta}\right)}{\alpha\left(\tau_{\epsilon}+\tau_{\zeta}+\tau_{\eta}+\tau_{\theta}\right)}\left(\sum_{j=1}^{N} a_{i j} s_{j}+b_{i} s\right),
$$


where

$$
\begin{aligned}
a_{i i} & \equiv \frac{\tau_{\eta}}{\tau_{\epsilon}+\tau_{\eta}+\tau_{\theta}}-A_{c}, \\
a_{i i_{r}} & \equiv-A_{r}, \\
a_{i j} & \equiv-A_{c} \quad \text { for } j \neq i, i_{r}, \\
b_{i} & \equiv \frac{\tau_{\theta}}{\tau_{\epsilon}+\tau_{\eta}+\tau_{\theta}}-B .
\end{aligned}
$$

Proceeding as in the proof of Proposition 6 , and using $\tau_{\eta_{i}}=\tau_{\eta}$ for all $i$, we find

$$
E\left(\left|x_{i}\right|\right)=\frac{\tau_{\zeta}\left(\tau_{\epsilon}+\tau_{\eta}+\tau_{\theta}\right)}{\alpha\left(\tau_{\epsilon}+\tau_{\zeta}+\tau_{\eta}+\tau_{\theta}\right)} \sqrt{\frac{2}{\pi}\left[\left(\sum_{j=1}^{N} a_{i j}+b_{i}\right)^{2} \sigma_{\epsilon}^{2}+\sum_{j=1}^{N} a_{i j}^{2} \sigma_{\eta}^{2}+b_{i}^{2} \sigma_{\theta}^{2}\right]},
$$

where $\sigma_{\eta}^{2} \equiv \frac{1}{\tau_{\eta}}$. Equations (A.85)-(A.88) imply that

$$
\sum_{j=1}^{N} a_{i j}+b_{i}=\frac{\tau_{\eta}}{\tau_{\epsilon}+\tau_{\eta}+\tau_{\theta}}-A_{r}-(N-1) A_{c}-B .
$$

Using (A.82) and setting $\tau_{\eta r}=\tau_{\eta c}=\tau_{\eta}, N_{r}=1, N_{c}=N-1$, and $\tau_{\xi}=(N-1) \tau_{\eta}$ (which is implied by (35) for $N_{r}=1$ and $N_{c}=N-1$ ), we find

$$
\sum_{j=1}^{N} a_{i j}+b_{i}=-\frac{(N-1) \tau_{\epsilon} \tau_{\eta}\left(\tau_{\epsilon}+\tau_{\zeta}+\tau_{\eta}+\tau_{\theta}\right)}{\left(\tau_{\epsilon}+\tau_{\eta}+\tau_{\theta}\right) \mathcal{G}},
$$

where

$$
\mathcal{G} \equiv(N-1)\left(\tau_{\epsilon}+\tau_{\eta}+\tau_{\theta}\right)\left(\tau_{\epsilon}+\tau_{\zeta}+N \tau_{\eta}+\tau_{\theta}\right)+\left(\tau_{\epsilon}+N \tau_{\eta}+\tau_{\theta}\right)\left(\tau_{\epsilon}+\tau_{\zeta}+\tau_{\eta}+\tau_{\theta}\right) .
$$

Using (A.84) and (A.88), and setting $\tau_{\eta r}=\tau_{\eta c}=\tau_{\eta}, N_{r}=1, N_{c}=N-1$ and $\tau_{\xi}=(N-1) \tau_{\eta}$, we likewise find

$$
b_{i}=\frac{(N-1) \tau_{\theta} \tau_{\eta}\left(\tau_{\epsilon}+\tau_{\zeta}+\tau_{\eta}+\tau_{\theta}\right)}{\left(\tau_{\epsilon}+\tau_{\eta}+\tau_{\theta}\right) \mathcal{G}},
$$

Equations (A.85)-(A.87) imply that

$$
\sum_{j=1}^{N} a_{i j}^{2}=\left(\frac{\tau_{\eta}}{\tau_{\epsilon}+\tau_{\eta}+\tau_{\theta}}-A_{c}\right)^{2}+A_{r}^{2}+(N-2) A_{c}^{2} .
$$


To compute this quantity, we note that for $\tau_{\eta r}=\tau_{\eta c}=\tau_{\eta}, N_{r}=1, N_{c}=N-1$ and $\tau_{\xi}=(N-1) \tau_{\eta}$, (36) implies that

$$
x=\frac{\tau_{\epsilon}+\tau_{\zeta}+\tau_{\eta}+\tau_{\theta}}{2\left(\tau_{\epsilon}+\tau_{\zeta}+\tau_{\theta}\right)+(N+1) \tau_{\eta}},
$$

and (32) and (33) become

$$
\begin{aligned}
A_{r} & =\frac{\tau_{\eta}\left[2\left(\tau_{\epsilon}+\tau_{\zeta}+\tau_{\theta}\right)+(N+1) \tau_{\eta}\right]}{\mathcal{G}}, \\
A_{c} & =\frac{\tau_{\eta}\left(\tau_{\epsilon}+\tau_{\zeta}+\tau_{\eta}+\tau_{\theta}\right)}{\mathcal{G}},
\end{aligned}
$$

respectively. Using (A.92) and (A.93), we find

$$
a_{i i}=\frac{\tau_{\eta}\left[(N-2)\left(\tau_{\epsilon}+\tau_{\eta}+\tau_{\theta}\right)\left(\tau_{\epsilon}+\tau_{\zeta}+N \tau_{\eta}+\tau_{\theta}\right)+(N-1) \tau_{\eta}\left(\tau_{\epsilon}+\tau_{\zeta}+\tau_{\eta}+\tau_{\theta}\right)\right]}{\left(\tau_{\epsilon}+\tau_{\eta}+\tau_{\theta}\right) \mathcal{G}},
$$

and

$$
\begin{aligned}
& \sum_{j \neq i}^{N} a_{i j}^{2}=\frac{\tau_{\eta}}{\mathcal{G}}\left[(N-1)\left(\tau_{\epsilon}+\tau_{\zeta}+\tau_{\eta}+\tau_{\theta}\right)^{2}\right. \\
& \left.+2(N-2)\left(\tau_{\epsilon}+\tau_{\zeta}+\tau_{\eta}+\tau_{\theta}\right)\left(\tau_{\epsilon}+\tau_{\zeta}+N \tau_{\eta}+\tau_{\theta}\right)+(N-2)\left(\tau_{\epsilon}+\tau_{\zeta}+N \tau_{\eta}+\tau_{\theta}\right)^{2}\right] .
\end{aligned}
$$

Using (A.90), (A.91), (A.94) and (A.95), we can write (A.89) as

$$
E\left(\left|x_{i}\right|\right)=\frac{\tau_{\zeta} \sqrt{2 \tau_{\eta}\left(\tau_{\epsilon}+\tau_{\eta}+\tau_{\theta}\right) \mathcal{N}_{c}}}{\alpha\left(\tau_{\epsilon}+\tau_{\zeta}+\tau_{\eta}+\tau_{\theta}\right) \mathcal{G} \sqrt{\pi}}
$$

where

$$
\begin{aligned}
\mathcal{N}_{c} \equiv & \left(\tau_{\epsilon}+N \tau_{\eta}+\tau_{\theta}\right)\left(\tau_{\epsilon}+\tau_{\zeta}+\tau_{\eta}+\tau_{\theta}\right)\left[(N-1)\left(\tau_{\epsilon}+\tau_{\zeta}+\tau_{\eta}+\tau_{\theta}\right)+2(N-2)\left(\tau_{\epsilon}+\tau_{\zeta}+N \tau_{\eta}+\tau_{\theta}\right)\right] \\
& +(N-2)(N-1)\left(\tau_{\epsilon}+\tau_{\eta}+\tau_{\theta}\right)\left(\tau_{\epsilon}+\tau_{\zeta}+N \tau_{\eta}+\tau_{\theta}\right)^{2} .
\end{aligned}
$$

We next compute the expected trading volume that the rational trader $i_{r}$ generates. The market-clearing equation (5) implies that

$$
x_{i_{r}}=-\sum_{j \neq i_{r}} x_{j}=-\frac{\tau_{\zeta}\left(\tau_{\epsilon}+\tau_{\eta}+\tau_{\theta}\right)}{\alpha\left(\tau_{\epsilon}+\tau_{\zeta}+\tau_{\eta}+\tau_{\theta}\right)}\left(\sum_{k=1}^{N} \sum_{j \neq i_{r}} a_{j k} s_{k}+\sum_{j \neq i_{r}} b_{j} s\right),
$$


and hence

$E\left(\left|x_{i_{r}}\right|\right)=\frac{\tau_{\zeta}\left(\tau_{\epsilon}+\tau_{\eta}+\tau_{\theta}\right)}{\alpha\left(\tau_{\epsilon}+\tau_{\zeta}+\tau_{\eta}+\tau_{\theta}\right)} \sqrt{\frac{2}{\pi}\left[\left(\sum_{k=1}^{N} \sum_{j \neq i_{r}} a_{j k}+\sum_{j \neq i_{r}} b_{j}\right)^{2} \sigma_{\epsilon}^{2}+\sum_{k=1}^{N}\left(\sum_{j \neq i_{r}} a_{j k}\right)^{2} \sigma_{\eta c}^{2}+\left(\sum_{j \neq i_{r}} b_{j}\right)^{2} \sigma_{\theta}^{2}\right]}$.

Equations (A.85)-(A.88) imply that

$$
\begin{aligned}
\sum_{k=1}^{N} \sum_{j \neq i_{r}} a_{j k}+\sum_{j \neq i_{r}} b_{j} & =(N-1)\left(\frac{\tau_{\eta}}{\tau_{\epsilon}+\tau_{\eta}+\tau_{\theta}}-(N-1) A_{c}-A_{r}-B\right) \\
& =-\frac{(N-1)^{2} \tau_{\epsilon} \tau_{\eta}\left(\tau_{\epsilon}+\tau_{\zeta}+\tau_{\eta}+\tau_{\theta}\right)}{\left(\tau_{\epsilon}+\tau_{\eta}+\tau_{\theta}\right) \mathcal{G}}
\end{aligned}
$$

where the second step follows from (A.90). Equation (A.88) implies that

$$
\begin{aligned}
\sum_{j \neq i_{r}} b_{j} & =(N-1)\left(\frac{\tau_{\theta}}{\tau_{\epsilon}+\tau_{\eta}+\tau_{\theta}}-B\right) \\
& =\frac{(N-1)^{2} \tau_{\theta} \tau_{\eta}\left(\tau_{\epsilon}+\tau_{\zeta}+\tau_{\eta}+\tau_{\theta}\right)}{\left(\tau_{\epsilon}+\tau_{\eta}+\tau_{\theta}\right) \mathcal{G}}
\end{aligned}
$$

where the second step follows from (A.91). Equations (A.86) and (A.87) imply that

$$
\begin{aligned}
\sum_{k=1}^{N}\left(\sum_{j \neq i_{r}} a_{j k}\right)^{2} & =(N-1)^{2} A_{r}^{2}+(N-1)\left(\frac{\tau_{\eta}}{\tau_{\epsilon}+\tau_{\eta}+\tau_{\theta}}-(N-1) A_{c}\right)^{2} \\
& =\frac{(N-1) \tau_{\eta}^{2}\left(\tau_{\epsilon}+\tau_{\zeta}+\tau_{\eta}+\tau_{\theta}\right)^{2}\left[(N-1)\left(\tau_{\theta}+\tau_{\eta}+\tau_{\theta}\right)^{2}+\left[\tau_{\eta}-(N-2)\left(\tau_{\epsilon}+\tau_{\theta}\right)\right]^{2}\right]}{\left(\tau_{\epsilon}+\tau_{\eta}+\tau_{\theta}\right)^{2} \mathcal{G}^{2}}
\end{aligned}
$$

where the second step follows from (A.92) and (A.93). Using (A.98), (A.99) and (A.100), we can write (A.97) as

$$
E\left(\left|x_{i_{r}}\right|\right)=\frac{\tau_{\zeta} \sqrt{2(N-1) \tau_{\eta}\left(\tau_{\epsilon}+N \tau_{\eta}+\tau_{\theta}\right) \mathcal{N}_{r}}}{\alpha \mathcal{G} \sqrt{\pi}},
$$

where

$$
\mathcal{N}_{r} \equiv(N-1)^{2}\left(\tau_{\epsilon}+\tau_{\eta}+\tau_{\theta}\right)-(N-2)\left(\tau_{\epsilon}+N \tau_{\eta}+\tau_{\theta}\right)
$$


Combining (23) with (A.96) and (A.101), we find that expected aggregate trading volume is larger when one trader is rational and $N-1$ traders are fully cursed than when all $N$ traders are fully cursed if

$$
\begin{aligned}
& N \frac{\sqrt{N-1}}{\left(\tau_{\epsilon}+\tau_{\zeta}+\tau_{\eta}+\tau_{\theta}\right) \sqrt{N}}<(N-1) \frac{\sqrt{\left(\tau_{\epsilon}+\tau_{\eta}+\tau_{\theta}\right) \mathcal{N}_{c}}}{\left(\tau_{\epsilon}+\tau_{\zeta}+\tau_{\eta}+\tau_{\theta}\right) \mathcal{G}}+\frac{\sqrt{(N-1)\left(\tau_{\epsilon}+N \tau_{\eta}+\tau_{\theta}\right) \mathcal{N}_{r}}}{\mathcal{G}} \\
& \Leftrightarrow \sqrt{N}<\frac{\sqrt{(N-1)\left(\tau_{\epsilon}+\tau_{\eta}+\tau_{\theta}\right) \mathcal{N}_{c}}}{\mathcal{G}}+\frac{\left(\tau_{\epsilon}+\tau_{\zeta}+\tau_{\eta}+\tau_{\theta}\right) \sqrt{\left(\tau_{\epsilon}+N \tau_{\eta}+\tau_{\theta}\right) \mathcal{N}_{r}}}{\mathcal{G}} .
\end{aligned}
$$

Equation (A.102) holds under the sufficient condition

$$
N<\frac{(N-1)\left(\tau_{\epsilon}+\tau_{\eta}+\tau_{\theta}\right) \mathcal{N}_{c}}{\mathcal{G}^{2}}+\frac{\left(\tau_{\epsilon}+\tau_{\zeta}+\tau_{\eta}+\tau_{\theta}\right)^{2}\left(\tau_{\epsilon}+N \tau_{\eta}+\tau_{\theta}\right) \mathcal{N}_{r}}{\mathcal{G}^{2}} .
$$

Multiplying both sides by $\mathcal{G}^{2}$, using the definitions of $\left(\mathcal{G}, \mathcal{N}_{c}, \mathcal{N}_{r}\right)$, and rearranging, we can write (A.103) as (38).

When $\tau_{\zeta}=\infty$, (38) becomes

$$
\frac{1}{N-1}\left(\frac{\tau_{\epsilon}+N \tau_{\eta}+\tau_{\theta}}{\tau_{\epsilon}+\tau_{\eta}+\tau_{\theta}}\right)^{2}+\left(\frac{2}{N-1}-1\right) \frac{\tau_{\epsilon}+N \tau_{\eta}+\tau_{\theta}}{\tau_{\epsilon}+\tau_{\eta}+\tau_{\theta}}+1<0 .
$$

Setting $y \equiv \frac{\tau_{\eta}}{\tau_{\epsilon}+\tau_{\theta}}$, we can write (A.104) as

$$
\begin{aligned}
& \frac{1}{N-1}\left(\frac{1+N y}{1+y}\right)^{2}-\frac{(N-3)(1+N y)}{(N-1)(1+y)}+1<0 \\
& 3-\left(N^{2}-6 N-1\right) y+(4 N-1) y^{2}<0 .
\end{aligned}
$$

The left-hand side of (A.105) converges to $-\infty$ when $N$ goes to $\infty$. Hence, (A.105) is satisfied for $N$ large enough.

Proof of Proposition 12. To derive the results for the rational case, we use (10)-(13) and set $\left(\chi_{i}, \alpha_{i}, \tau_{\eta_{i}}, \tau_{z_{i}}, A_{i}, C_{i}\right)=\left(0, \alpha, \tau_{\eta}, \tau_{z}, A, C\right)$ for all $i$. Equations (10)-(13) yield

$$
\begin{aligned}
\tau_{\xi_{i}} & =\frac{(N-1) \tau_{\eta} \tau_{z}}{\tau_{z}+\frac{C^{2}}{A^{2}} \tau_{\eta}} \equiv \tau_{\xi} \quad \text { for all } i, \\
A & =\frac{\tau_{\eta}+\tau_{\xi}}{N\left(\tau_{\epsilon}+\tau_{\eta}+\tau_{\theta}+\tau_{\xi}\right)}, \\
B & =\frac{\tau_{\theta}}{\tau_{\epsilon}+\tau_{\eta}+\tau_{\theta}+\tau_{\xi}}, \\
\frac{C}{A} & =\alpha \frac{\tau_{\epsilon}+\tau_{\zeta}+\tau_{\eta}+\tau_{\theta}+\tau_{\xi}}{\tau_{\zeta} \tau_{\eta}},
\end{aligned}
$$


respectively. Substituting $\tau_{\xi}$ from (A.106) to (A.107)-(A.109), we find (40)-(42), respectively. Eq. (42) is cubic in $\frac{C}{A}$, and hence has at least one solution. Any of its solutions satisfies

$$
\frac{C}{A} \tau_{\zeta} \tau_{\eta}-\alpha\left(\tau_{\epsilon}+\tau_{\zeta}+\tau_{\eta}+\tau_{\theta}\right)>0
$$

and hence is positive. The derivative of the left-hand side of (42) with respect to $\frac{C}{A}$ is

$$
2 \frac{C}{A} \tau_{\eta}\left(\frac{C}{A} \tau_{\zeta} \tau_{\eta}-\alpha\left(\tau_{\epsilon}+\tau_{\zeta}+\tau_{\eta}+\tau_{\theta}\right)\right)+\left(\tau_{z}+\frac{C^{2}}{A^{2}} \tau_{\eta}\right) \tau_{\zeta} \tau_{\eta},
$$

and is positive at any solution of (42) because of (A.110). Therefore, (42) has a unique solution.

To derive the results for the fully cursed case, we use (11)-(13) and set $\left(\chi_{i}, \alpha_{i}, \tau_{\eta_{i}}, \tau_{z_{i}}, A_{i}, C_{i}\right)=$ $\left(1, \alpha, \tau_{\eta}, \tau_{z}, A, C\right)$ for all $i$. Equations (11)-(13) yield

$$
\begin{aligned}
A & =\frac{\tau_{\eta}}{N\left(\tau_{\epsilon}+\tau_{\eta}+\tau_{\theta}\right)}, \\
B & =\frac{\tau_{\theta}}{\tau_{\epsilon}+\tau_{\eta}+\tau_{\theta}}, \\
C & =A \alpha \frac{\tau_{\epsilon}+\tau_{\zeta}+\tau_{\eta}+\tau_{\theta}}{\tau_{\zeta} \tau_{\eta}},
\end{aligned}
$$

respectively. The coefficients $(A, B, C)$ coincide with those in (40)-(42) for $\tau_{\zeta}=0$.

Proof of Proposition 13. To show the result for regression (21), we note that $\gamma$ is proportional to

$$
\operatorname{Cov}(d-p, s)=(1-N A-B) \sigma_{\epsilon}^{2}-B \sigma_{\theta}^{2},
$$

where the equality follows from (1), (2) and (39). Substituting $(A, B)$ from Proposition 12, we find that

$$
\operatorname{Cov}(d-p, s)=\left(1-\frac{\tau_{\eta}+\frac{(N-1) \tau_{\eta} \tau_{z}}{\tau_{z}+\frac{C^{2}}{A^{2}} \tau_{\eta}}+\tau_{\theta}}{\tau_{\epsilon}+\tau_{\eta}+\tau_{\theta}+\frac{(N-1) \tau_{\eta} \tau_{z}}{\tau_{z}+\frac{C^{2}}{A^{2}} \tau_{\eta}}}\right) \sigma_{\epsilon}^{2}-\frac{\tau_{\theta}}{\tau_{\epsilon}+\tau_{\eta}+\tau_{\theta}+\frac{(N-1) \tau_{\eta} \tau_{z}}{\tau_{z}+\frac{C^{2}}{A^{2}} \tau_{\eta}}} \sigma_{\theta}^{2}=0
$$

when all traders are rational, and

$$
\operatorname{Cov}(d-p, s)=\left(1-\frac{\tau_{\eta}+\tau_{\theta}}{\tau_{\epsilon}+\tau_{\eta}+\tau_{\theta}}\right) \sigma_{\epsilon}^{2}-\frac{\tau_{\theta}}{\tau_{\epsilon}+\tau_{\eta}+\tau_{\theta}} \sigma_{\theta}^{2}=0
$$

when they are fully cursed. 
To show the result for regression (22), we likewise note that $\gamma$ is proportional to

$$
\begin{aligned}
\operatorname{Cov}(d-p, p-\bar{d}) & =(1-N A-B)(N A+B) \sigma_{\epsilon}^{2}-N A^{2} \sigma_{\eta}^{2}-B^{2} \sigma_{\theta}^{2}-N C^{2} \sigma_{z}^{2} \\
& =(1-N A-B)(N A+B) \sigma_{\epsilon}^{2}-N A^{2}\left(\sigma_{\eta}^{2}+\frac{C^{2}}{A^{2}} \sigma_{z}^{2}\right)-B^{2} \sigma_{\theta}^{2},
\end{aligned}
$$

where $\sigma_{z}^{2} \equiv \frac{1}{\tau_{z}}$. Substituting $(A, B, C)$ from Proposition 12, we find that

$$
\begin{array}{rl}
\operatorname{Cov}(d-p, p-\bar{d}) & =\frac{\tau_{\eta}+\frac{(N-1) \tau_{\eta} \tau_{z}}{\tau_{z}+\frac{C^{2}}{A^{2}} \tau_{\eta}}}{\left(\tau_{\epsilon}+\tau_{\eta}+\tau_{\theta}+\frac{(N-1) \tau_{\eta} \tau_{z}}{\tau_{z}+\frac{C^{2}}{A^{2}} \tau_{\eta}}\right)^{2}}-\frac{\left(\tau_{\eta}+\frac{(N-1) \tau_{\eta} \tau_{z}}{\tau_{z}+\frac{C^{2}}{A^{2}} \tau_{\eta}}\right)^{2}\left(\sigma_{\eta}^{2}+\frac{C^{2}}{A^{2}} \sigma_{z}^{2}\right)}{N\left(\tau_{\epsilon}+\tau_{\eta}+\tau_{\theta}+\frac{(N-1) \tau_{\eta} \tau_{z}}{\tau_{z}+\frac{C^{2}}{A^{2}} \tau_{\eta}}\right)^{2}} \\
& \left.=\frac{\tau_{\eta}+\frac{(N-1) \tau_{\eta} \tau_{z}}{\tau_{z}+\frac{C^{2}}{A^{2}} \tau_{\eta}}}{\left(\tau_{\eta}+\frac{(N-1) \tau_{\eta} \tau_{z}}{\tau_{z}+\frac{C^{2}}{A^{2}} \tau_{\eta}}\right)\left(\tau_{z}+\frac{C^{2}}{A^{2}} \tau_{\eta}\right)}\right) \\
\left.N \tau_{\eta}+\tau_{\theta}+\frac{(N-1) \tau_{\eta} \tau_{z}}{\tau_{z}+\frac{C^{2}}{A^{2}} \tau_{\eta}}\right)^{2} & 1-\frac{\left(\tau^{2}\right.}{\left(\tau_{\eta}+\frac{(N-1) \tau_{\eta} \tau_{z}}{\tau_{z}+\frac{C^{2}}{A^{2}} \tau_{\eta}}\right) \frac{C^{2} \tau_{\eta}}{N A^{2} \tau_{z}}} \\
& =-\frac{\left(\tau_{\epsilon}+\tau_{\eta}+\tau_{\theta}+\frac{(N-1) \tau_{\eta} \tau_{z}}{\tau_{z}+\frac{C^{2}}{A^{2}} \tau_{\eta}}\right)^{2}}{\left(\tau_{\eta}\right.}<0
\end{array}
$$

when all traders are rational, and

$$
\begin{aligned}
\operatorname{Cov}(d-p, p-\bar{d}) & =\frac{\tau_{\eta}}{\left(\tau_{\epsilon}+\tau_{\eta}+\tau_{\theta}\right)^{2}}-\frac{\tau_{\eta}^{2}\left(\sigma_{\eta}^{2}+\frac{C^{2}}{A^{2}} \sigma_{z}^{2}\right)}{N\left(\tau_{\epsilon}+\tau_{\eta}+\tau_{\theta}\right)^{2}} \\
& =\frac{\tau_{\eta}}{\left(\tau_{\epsilon}+\tau_{\eta}+\tau_{\theta}\right)^{2}}\left(1-\frac{\tau_{\eta}\left(\tau_{z}+\frac{C^{2}}{A^{2}} \tau_{\eta}\right)}{N \tau_{\eta} \tau_{z}}\right) \\
& =\frac{(N-1) \tau_{\eta}}{N\left(\tau_{\epsilon}+\tau_{\eta}+\tau_{\theta}\right)^{2}}\left(1-\frac{\alpha^{2}\left(\tau_{\epsilon}+\tau_{\zeta}+\tau_{\eta}+\tau_{\theta}\right)^{2}}{(N-1) \tau_{\zeta}^{2} \tau_{\eta} \tau_{z}}\right)
\end{aligned}
$$

when they are fully cursed. The last step in the derivation of (A.111) follows from (42). Equation (A.111) yields (43).

Proof of Proposition 14. We first determine expected trading volume when all traders are rational. When $\left(A_{i}, C_{i}\right)=(A, C)$ for all $i$, the signal $\epsilon+\xi_{i}$ defined by $(9)$ takes the form

$$
\epsilon+\xi_{i}=\frac{\sum_{j \neq i} s_{j}-\frac{C}{A} \sum_{j \neq i} z_{j}}{N-1} .
$$


Substituting $p$ from (39) into (A.13), and using symmetry, $\chi_{i}=0$ and (A.112), we can write the quantity that trader $i$ trades in equilibrium as

$$
x_{i}=\frac{\tau_{\zeta}\left(\tau_{\epsilon}+\tau_{\eta}+\tau_{\theta}+\tau_{\xi}\right)}{\alpha\left(\tau_{\epsilon}+\tau_{\zeta}+\tau_{\eta}+\tau_{\theta}+\tau_{\xi}\right)}\left(\sum_{j=1}^{N} a_{i j} s_{j}+b_{i} s+\sum_{j=1}^{N} c_{i j} z_{j}\right)
$$

where

$$
\begin{aligned}
a_{i i} & \equiv \frac{\tau_{\eta}}{\tau_{\epsilon}+\tau_{\eta}+\tau_{\theta}+\tau_{\xi}}-A, \\
a_{i j} & \equiv \frac{\tau_{\xi}}{(N-1)\left(\tau_{\epsilon}+\tau_{\eta}+\tau_{\theta}+\tau_{\xi}\right)}-A \quad \text { for } j \neq i, \\
b_{i} & \equiv \frac{\tau_{\theta}}{\tau_{\epsilon}+\tau_{\eta}+\tau_{\theta}+\tau_{\xi}}-B, \\
c_{i i} & \equiv C-\frac{\alpha\left(\tau_{\epsilon}+\tau_{\zeta}+\tau_{\eta}+\tau_{\theta}+\tau_{\xi}\right)}{\tau_{\zeta}\left(\tau_{\epsilon}+\tau_{\eta}+\tau_{\theta}+\tau_{\xi}\right)}, \\
c_{i j} & \equiv C-\frac{C \tau_{\xi}}{(N-1) A\left(\tau_{\epsilon}+\tau_{\eta}+\tau_{\theta}+\tau_{\xi}\right)} \quad \text { for } j \neq i .
\end{aligned}
$$

Proceeding as in the proof of Proposition (6), we find

$$
E\left(\left|x_{i}\right|\right)=\frac{\tau_{\zeta}\left(\tau_{\epsilon}+\tau_{\eta}+\tau_{\theta}+\tau_{\xi}\right)}{\alpha\left(\tau_{\epsilon}+\tau_{\zeta}+\tau_{\eta}+\tau_{\theta}+\tau_{\xi}\right)} \sqrt{\frac{2}{\pi}\left[\left(\sum_{j=1}^{N} a_{i j}+b_{i}\right)^{2} \sigma_{\epsilon}^{2}+\sum_{j=1}^{N} a_{i j}^{2} \sigma_{\eta}^{2}+b_{i}^{2} \sigma_{\theta}^{2}+\sum_{j=1}^{N} c_{i j}^{2} \sigma_{z}^{2}\right]}
$$

Substituting $(A, B, C)$ from (A.107)-(A.109) into (A.114)-(A.118), we find

$$
\begin{aligned}
a_{i i} & =\frac{(N-1) \tau_{\eta}-\tau_{\xi}}{N\left(\tau_{\epsilon}+\tau_{\eta}+\tau_{\theta}+\tau_{\xi}\right)}, \\
a_{i j} & =-\frac{(N-1) \tau_{\eta}-\tau_{\xi}}{N(N-1)\left(\tau_{\epsilon}+\tau_{\eta}+\tau_{\theta}+\tau_{\xi}\right)} \text { for } j \neq i, \\
b_{i} & =0, \\
c_{i i} & =-\frac{\alpha\left(\tau_{\epsilon}+\tau_{\zeta}+\tau_{\eta}+\tau_{\theta}+\tau_{\xi}\right)\left[(N-1) \tau_{\eta}-\tau_{\xi}\right]}{N \tau_{\zeta} \tau_{\eta}\left(\tau_{\epsilon}+\tau_{\eta}+\tau_{\theta}+\tau_{\xi}\right)}, \\
c_{i j} & =\frac{\alpha\left(\tau_{\epsilon}+\tau_{\zeta}+\tau_{\eta}+\tau_{\theta}+\tau_{\xi}\right)\left[(N-1) \tau_{\eta}-\tau_{\xi}\right]}{N(N-1) \tau_{\zeta} \tau_{\eta}\left(\tau_{\epsilon}+\tau_{\eta}+\tau_{\theta}+\tau_{\xi}\right)} \text { for } j \neq i, \\
\sum_{j=1}^{N} a_{i j}+b_{i} & =0 .
\end{aligned}
$$


Substituting into (A.119), we find

$$
\begin{aligned}
E\left(\left|x_{i}\right|\right) & =\sqrt{\frac{2\left[(N-1) \tau_{\eta}-\tau_{\xi}\right]^{2}}{\pi N(N-1) \tau_{\eta}}\left(\frac{\tau_{\zeta}^{2}}{\alpha^{2}\left(\tau_{\epsilon}+\tau_{\zeta}+\tau_{\eta}+\tau_{\theta}+\tau_{\xi}\right)^{2}}+\frac{1}{\tau_{\eta} \tau_{z}}\right)} \\
& =\sqrt{\frac{2\left[(N-1) \tau_{\eta}-\tau_{\xi}\right]^{2}}{\pi N(N-1) \tau_{\eta}}\left(\frac{A^{2}}{C^{2} \tau_{\eta}^{2}}+\frac{1}{\tau_{\eta} \tau_{z}}\right)} \\
& =\sqrt{\frac{2(N-1)^{2} \tau_{\eta}^{2}\left(1-\frac{\tau_{z}}{\tau_{z}+\frac{C^{2}}{A^{2}} \tau_{\eta}}\right)^{2}}{\pi N(N-1) \tau_{\eta}}\left(\frac{A^{2}}{C^{2} \tau_{\eta}^{2}}+\frac{1}{\tau_{\eta} \tau_{z}}\right)} \\
& =\sqrt{\frac{2(N-1) \frac{C^{2}}{A^{2}} \tau_{\eta}}{\pi N \tau_{z}\left(\tau_{z}+\frac{C^{2}}{A^{2}} \tau_{\eta}\right)}},
\end{aligned}
$$

where the second step follows from (A.109) and the third from (A.106).

Equation (A.120) implies that volume is increasing in $N$ if $\frac{C}{A}$ is increasing in $N$. In the proof of Proposition 12 we show that the derivative of the left-hand side of (42) with respect to $\frac{C}{A}$ is positive at the solution $\frac{C}{A}$. Since the derivative with respect to $N$ is negative, $\frac{C}{A}$ is increasing in $N$.

To show that volume is inverse hump-shaped in $\tau_{\eta}$, we set $\omega \equiv \frac{C}{A} \sqrt{\tau_{\eta}}$ and write (42) as

$$
\left(\tau_{z}+w^{2}\right)\left(\omega \tau_{\zeta} \sqrt{\tau_{\eta}}-\alpha\left(\tau_{\epsilon}+\tau_{\zeta}+\tau_{\eta}+\tau_{\theta}\right)\right)-\alpha(N-1) \tau_{\eta} \tau_{z}=0
$$

and (A.120) as

$$
E\left(\left|x_{i}\right|\right)=\sqrt{\frac{2(N-1) \omega^{2}}{\pi N \tau_{z}\left(\tau_{z}+\omega^{2}\right)}} .
$$

Eq. (A.122) implies that the effects of $\tau_{\eta}$ on volume and $\omega$ have the same sign. The derivative of the left-hand side of (A.121) with respect to $\omega$ is equal to $\sqrt{\tau_{\eta}}$ times the same derivative with respect to $\frac{C}{A}$. Therefore, it is positive at the solution $\omega$. The derivative with respect to $\tau_{\eta}$ is

$$
\left(\tau_{z}+w^{2}\right)\left(\frac{\omega \tau_{\zeta}}{2 \sqrt{\tau_{\eta}}}-\alpha\right)-\alpha(N-1) \tau_{z}
$$

and is equal to

$$
\begin{aligned}
& \frac{1}{\tau_{\eta}}\left[\left(\tau_{z}+w^{2}\right)\left(\frac{1}{2} \omega \tau_{\zeta} \sqrt{\tau_{\eta}}-\alpha \tau_{\eta}\right)-\alpha(N-1) \tau_{\eta} \tau_{z}\right] \\
& =\frac{1}{\tau_{\eta}}\left[\left(\tau_{z}+w^{2}\right)\left(\frac{1}{2} \omega \tau_{\zeta} \sqrt{\tau_{\eta}}-\alpha \tau_{\eta}\right)-\left(\tau_{z}+w^{2}\right)\left(\omega \tau_{\zeta} \sqrt{\tau_{\eta}}-\alpha\left(\tau_{\epsilon}+\tau_{\zeta}+\tau_{\eta}+\tau_{\theta}\right)\right)\right]
\end{aligned}
$$


at the solution $w$. It is negative if and only if

$$
\omega>\frac{2 \alpha\left(\tau_{\epsilon}+\tau_{\zeta}+\tau_{\theta}\right)}{\tau_{\zeta} \sqrt{\tau_{\eta}}}
$$

This condition is equivalent to the left-hand side of (A.121) being negative for $\omega=\frac{2 \alpha\left(\tau_{\epsilon}+\tau_{\zeta}+\tau_{\theta}\right)}{\tau_{\zeta} \sqrt{\tau_{\eta}}}$. We can write the latter condition as

$$
\left[\tau_{\zeta}^{2} \tau_{\eta} \tau_{z}+4 \alpha^{2}\left(\tau_{\epsilon}+\tau_{\zeta}+\tau_{\theta}\right)^{2}\right]\left(\tau_{\epsilon}+\tau_{\zeta}+\tau_{\theta}-\tau_{\eta}\right)-(N-1) \tau_{\zeta}^{2} \tau_{\eta}^{2} \tau_{z}<0
$$

The left-hand side of (A.123) is a quadratic function of $\tau_{\eta}$ that is positive for $\tau_{\eta}=0$ and goes to $-\infty$ when $\tau_{\eta}$ goes to $\infty$. Therefore, there exists a unique $\tau_{\eta}^{*}>0$ such that (A.123) holds for $\tau_{\eta}>\tau_{\eta}^{*}$ and the opposite inequality holds for $\tau_{\eta}<\tau_{\eta}^{*}$. Volume and $\omega$ are thus decreasing in $\tau_{\eta}$ for $\tau_{\eta}<\tau_{\eta}^{*}$ and increasing in $\tau_{\eta}$ for $\tau_{\eta}>\tau_{\eta}^{*}$, i.e., are inverse hump-shaped in $\tau_{\eta}$.

We next determine expected trading volume when all traders are fully cursed. Substituting $p$ from (39) into (A.13), and using symmetry and $\chi_{i}=1$, we can write the quantity that trader $i$ trades in equilibrium as

$$
x_{i}=\frac{\tau_{\zeta}\left(\tau_{\epsilon}+\tau_{\eta}+\tau_{\theta}\right)}{\alpha\left(\tau_{\epsilon}+\tau_{\zeta}+\tau_{\eta}+\tau_{\theta}\right)}\left(\sum_{j=1}^{N} a_{i j} s_{j}+b_{i} s+\sum_{j=1}^{N} c_{i j} z_{j}\right),
$$

where

$$
\begin{aligned}
a_{i i} & \equiv \frac{\tau_{\eta}}{\tau_{\epsilon}+\tau_{\eta}+\tau_{\theta}}-A, \\
a_{i j} & \equiv-A \quad \text { for } j \neq i \\
b_{i} & \equiv \frac{\tau_{\theta}}{\tau_{\epsilon}+\tau_{\eta}+\tau_{\theta}}-B \\
c_{i i} & \equiv C-\frac{\alpha\left(\tau_{\epsilon}+\tau_{\zeta}+\tau_{\eta}+\tau_{\theta}\right)}{\tau_{\zeta}\left(\tau_{\epsilon}+\tau_{\eta}+\tau_{\theta}\right)}, \\
c_{i j} & \equiv C \quad \text { for } j \neq i .
\end{aligned}
$$

Proceeding as in the proof of Proposition (6), we find

$$
E\left(\left|x_{i}\right|\right)=\frac{\tau_{\zeta}\left(\tau_{\epsilon}+\tau_{\eta}+\tau_{\theta}\right)}{\alpha\left(\tau_{\epsilon}+\tau_{\zeta}+\tau_{\eta}+\tau_{\theta}\right)} \sqrt{\frac{2}{\pi}\left[\left(\sum_{j=1}^{N} a_{i j}+b_{i}\right)^{2} \sigma_{\epsilon}^{2}+\sum_{j=1}^{N} a_{i j}^{2} \sigma_{\eta}^{2}+b_{i}^{2} \sigma_{\theta}^{2}+\sum_{j=1}^{N} c_{i j}^{2} \sigma_{z}^{2}\right]} .
$$


Substituting $(A, B, C)$ from (A.107)-(A.109) for $\tau_{z}=0$ into (A.125)-(A.129), we find

$$
\begin{aligned}
a_{i i} & =\frac{(N-1) \tau_{\eta}}{N\left(\tau_{\epsilon}+\tau_{\eta}+\tau_{\theta}\right)}, \\
a_{i j} & =-\frac{\tau_{\eta}}{N\left(\tau_{\epsilon}+\tau_{\eta}+\tau_{\theta}\right)} \quad \text { for } j \neq i, \\
b_{i} & =0, \\
c_{i i} & =-\frac{(N-1) \alpha\left(\tau_{\epsilon}+\tau_{\zeta}+\tau_{\eta}+\tau_{\theta}\right)}{N \tau_{\zeta}\left(\tau_{\epsilon}+\tau_{\eta}+\tau_{\theta}\right)}, \\
c_{i j} & =\frac{\alpha\left(\tau_{\epsilon}+\tau_{\zeta}+\tau_{\eta}+\tau_{\theta}\right)}{N \tau_{\zeta}\left(\tau_{\epsilon}+\tau_{\eta}+\tau_{\theta}\right)} \text { for } j \neq i, \\
\sum_{j=1}^{N} a_{i j}+b_{i} & =0 .
\end{aligned}
$$

Substituting into (A.130), we find

$$
E\left(\left|x_{i}\right|\right)=\sqrt{\frac{2(N-1)}{\pi N}\left(\frac{\tau_{\zeta}^{2} \tau_{\eta}}{\alpha^{2}\left(\tau_{\epsilon}+\tau_{\zeta}+\tau_{\eta}+\tau_{\theta}\right)^{2}}+\frac{1}{\tau_{z}}\right)} .
$$

The square of the right-hand side of (A.131) is equal to its counterpart without endowment shocks (the square of the right-hand side of (23)) plus a constant. Therefore, (A.131) is increasing in $N$ and is hump-shaped in $\tau_{\eta}$.

Proof of Proposition 15. We first determine traders' demand functions assuming that the price takes the form (44). The information $I_{i}$ of trader $i$ consists of his private signal $s_{i}$, the public signal $s$, and the signal $\epsilon+\xi_{i}$ that is revealed from the price. Since all private signals enter the price with the same coefficient, (9) implies that

$$
\epsilon+\xi_{i}=\frac{\sum_{j \neq i} s_{j}}{N-1} \Rightarrow \xi_{i}=\frac{\sum_{j \neq i} \eta_{j}}{N-1} .
$$

To compute the distribution of $\epsilon$ conditional on $I_{i}$, we use Lemma A.1 with $x=\epsilon, K=3$, $\left\{y_{j}\right\}_{j=1,2,3}=\left(\eta_{i}, \eta, \xi_{i}\right)$, and trader $i$ 's assessments of precision. Combining with (A.7) and (A.8), and setting $\left(\chi_{i}, I_{i r}\right)=\left(0, I_{i}\right)$ in $(8)$, we can write the demand of trader $i$ as

$$
x_{i}=\frac{\bar{d}+\frac{\kappa \tau_{\eta}}{\tau_{\epsilon}+\kappa \tau_{\eta}+\tau_{\theta}+(N-1) \gamma \tau_{\eta}} s_{i}+\frac{\tau_{\theta}}{\tau_{\epsilon}+\kappa \tau_{\eta}+\tau_{\theta}+(N-1) \gamma \tau_{\eta}} s+\frac{(N-1) \gamma \tau_{\eta}}{\tau_{\epsilon}+\kappa \tau_{\eta}+\tau_{\theta}+(N-1) \gamma \tau_{\eta}}\left(\epsilon+\xi_{i}\right)-p}{\alpha\left(\frac{1}{\tau_{\epsilon}+\kappa \tau_{\eta}+\tau_{\theta}+(N-1) \gamma \tau_{\eta}}+\frac{1}{\tau_{\zeta}}\right)} .
$$


We next substitute (A.133) into the market-clearing condition (5), substituting also $p$ from (44) and $\epsilon+\xi_{i}$ from (A.132). This yields an equation that is linear in $\left(\sum_{i=1}^{N} s_{i}, s\right)$. Identifying terms in $\sum_{i=1}^{N} s_{i}$ and $s$ yields (45) and (46), respectively. The comparative statics of $A$ and $B$ follow from (45) and (46).

Proof of Proposition 16. Substituting $p$ from (44) into (A.133) and using (45), (46) and (A.132), we can write the quantity that trader $i$ trades in equilibrium as

$$
x_{i}=\frac{\tau_{\zeta}}{\alpha\left[\tau_{\epsilon}+\tau_{\zeta}+[(N-1) \gamma+\kappa] \tau_{\eta}+\tau_{\theta}\right]} \sum_{j=1}^{N} a_{i j} s_{j},
$$

where

$$
\begin{aligned}
a_{i i} & \equiv \frac{N-1}{N}(\kappa-\gamma) \tau_{\eta}, \\
a_{i j} & \equiv-\frac{1}{N}(\kappa-\gamma) \tau_{\eta} \quad \text { for } j \neq i .
\end{aligned}
$$

Proceeding as in the proof of Proposition (6), we find

$$
E\left(\left|x_{i}\right|\right)=\frac{\tau_{\zeta}}{\alpha\left[\tau_{\epsilon}+\tau_{\zeta}+[(N-1) \gamma+\kappa] \tau_{\eta}+\tau_{\theta}\right]} \sqrt{\frac{2}{\pi}\left[\left(\sum_{j=1}^{N} a_{i j}\right)^{2} \sigma_{\epsilon}^{2}+\sum_{j=1}^{N} a_{i j}^{2} \sigma_{\eta}^{2}\right]} .
$$

Substituting $\left\{a_{i j}\right\}_{j=1, . ., N}$ from (A.135) and (A.136) into (A.137), we find (47). The asymptotic behavior of $E\left(\left|x_{i}\right|\right)$ and of aggregate volume $N E\left(\left|x_{i}\right|\right)$ follows from (A.137).

Proof of Proposition 17. The proposition follows by setting $\gamma=0$ in (47) since full cursedness is equivalent to extreme contemptuousness. 